\title{
Sulle cause delle " acque alte " nell'Adriatico settentrionale, con particolare riguardo alla Laguna di Venezia
}

\author{
P. CALOI $(*)$ \\ Ricevuto il 5 Gennain 1973 \\ "Desponsamus te, mare, in signum veri \\ perpetuigue domimi $\mathrm{n}$. \\ (Formula dogule per lo : Sprosalizio del mare )
}

\begin{abstract}
Riassunto. - È opinione diffusa fra gli esperti che le canse dell'aumentata frequenza rlelle "acque alte n nella Laguna di Venezia siano limitate allo sprofondamento in atto nella zona (associato a tisordinati emungimenti di acque dal sottosuolo), e al bradisismo clie interessa la costa oceitentale tell'alto Aslriatico.

In realtà, l'alto Adriatico si trova in fase di lentissimo ricmpimento, provocato Ilall'enorme quantità di prodotti d'erosione, in esso eonvogliata dalle diecine di fiumi che vi sfociano; quantità che ragrgiunge proporzioni eccezionali in epoche di piena, specialmente da parte del Po. L'innalzamento del fondo che ne consegue, nel complesso supera l'eftetio dell'enstatigmo e del bradisismo. Pertanto, l'equilibrio idrodinamico del golfo di Venezia è soggetto a continua alterazione, nel senso che assottigliantlosi - sia pure lentissimamente - lo spessore rlelle acque, queste sono costrettc (a parità di altre condizioni) as un lentissimo aumento delle ampiezze dei movimenti liberi, forzati o progressivi; nonché al lentissimo progressivo aumento del periodo delle oscillazioni libere che le interessano. Qnest'ultimo aspetto è particolarmente preoceupante, se si pensa che le oseillazioni libere di $1 l^{\text {ha }}, 7 \mathrm{ea}$
\end{abstract}

(*) Istituto Nazionale di Gcofisica, Roma. 
e di $23^{\text {h }}$ ea tendono - sia pure con estrema lentezza - verso il ritmo delle maree semidiurne e diurne, accentuando nel tempo l'efietto di risonanza.

I. stato constatato che, a partire dal 1940, la temperatura media dell'aria sulla Terra - in leggero aumento dal 1880 - ha segnato un'inversione di tendenza; se ciò ha portato ad un'attenuazione dell'clictto cli eustatismo, ha jerò provocato un progressivo aumento delle precipitazionni atmosferiehe, il che si trarluce in un'acentuazione dell'efficacia delle eause naturali associate alle acque alte; non ultima la " lievitazione s del mare per diminuita salinità accompagnata da diminuzione di densità, di viscosità molecolare e di tensione superficiale -, responsabile di effetti isostatici ed agevolazione e rinforzo dei moti del mare per fenomeni di risonanza.

In ogni modo, scmbra che il motivo preponderante delle acque alte nel golfo di Venezia, vada ricercato nel transito di zone di bassa jressione, dal largo verso la costa; la corrispondente intumescenza marina, nel suo procedere lungo il piano inclinato costituito dal fondo, raggiunto il punto di risonanza (eiò che può frequentemente verificarsi nell'alto Arlriatico), ivi subisce un notevole rinforzo, che supera talvolta di parechie unità l'cfictto statico, e tale ampiezza maggiorata conduce fino alla ensta. L'cfficacia di questo meccanismo è tanto maggiore quanto più dolce è l'inclinazione del fondo. Pertanto, in conseguenza dell'abbassamento del lato oceidentale del golfo di Venezia, particolarmente sensibile in corrispondenza del Delta, a partire dal 1951 - a parità di altre condlizioni - è andata via via csaltandosi l'inciłlenza di questo fenomeno sulle acque alte.

Si prova che l'alto $\Lambda$ driation (o sue parti) può rendersi estremamente sensibile a sollecitazioni da parte di variazioni di pressione in transito e che fenomeni di risonanza possono verificarsi sia per confluenze periodali, sia per coincirlenze cinetiche. In ogni caso, le variazioni di pressione vanno ritenute di gran lunga le più ellicienti cause di acque alte nelle lagune venete.

Contrariamente ad un'opinione molto diffusa, il vento non ha azione determinante nella formazione rli acque alte; il suo intervento, agisec sempre da causa concomitante.

Naturalmente, esula dagli scopi di questo lavoro la ricerca di rimedi per la salvaguarlia di Venezia. Si può solo sottolineare che l'aggravamento della sua situazione è legato - per quanto è stato sopra rieordato - ad un'eventriale ulteriore cedimento del sottosuolo. In un precedente lavoro, ho richiamato l'attenzione sulla sit uazione altrettanto allarmante che si era verificata a Wilmington (Long Beach), dove la locale base della Marina militare U.S.A. come conseguenza dell'estrazione di petrolio nel vicino giacimento di Long Beaclı - era venuta a trovarsi, nel 1959 , con le banehine prossime alla som. mersinne. I'intervento di cospicue immissioni d'acqua nel sottosuolo, in tutta la zona interessata dall'ablussamento, valse non solo ad arrestare il preoccupante fenomeno, ma altresí a riportare la superficie esterna verso le primitive posizioni, nei riguardi del mare. Una accurata, ben ponderata azione di pressurizzazione sotterranea nella zona della laguna, dovrebbe sortire risultati altrettanto apparezzabili nei confronti di Venczia.

Certo, stanno per invertirsi le parti nella formula dello sposalizio di Venczia eol mare: nulla deve restare intentato, allo scopo di evitare che tanta iattura venga portata a compimento. 
1. - "ACQUe atte" in UN bacino a CONfigurazione costante.

Supponiamo che le caratteristiche morfologiche dell'alto $\Lambda$ driatico siano rimaste immutate attraverso l'ultimo secolo. In questa jpotesi prescindendo dalla marea astronomica - da ritenersi pure inmutata nel suo verificarsi, durante il lasso di tempo considerato - a quali cause vanno attribuite le acque alte osservate nelle lagune venete? Tali cause, evicientemente si identificano con intcrazioni fra atmosfera ed iỏrosiera. Di tempo in tempo, la mia attenzione è stata a più ripresc attratta da tali fenomeni. Gli aspetti fondamentali che li caratterizzano, sono stati da me riassunti in un lavoro del 1963, al quale rimando il lettore $\left({ }^{1}\right)$.

Qui mi preme sottolineare che movimenti delle acque di eccezionale anpiezza, sono sempre legati a fenomeni di risonanza, che insorgono durante il propagarsi di più o meno vaste perturbazioni della pressione atmosferica, sopra il bacino idrico sottostante. Due sono le principali condizioni di risonanza: velocità di propagazione (iella perturbazione atmosferica tendente alla velocità delle onde libere del mare su cui transita; periodo portante della variazione di pressione tendente a coincidere con il periodo proprio di un'oscillazione libera del sottoposto bacino.

Con la prima causa, p. es., si spiega il formarsi della sessa del golfo di Trieste (e, quindi, dell'oscillazione libera trasversale dell'alto Adriatico). Ho già trattato l'argomento in più occasioni. Qui mi basterà richiamare la formula, che sintetizza l'andiamento del fenomeno. Se consideriamo un canale chiuso all'estremo $x=0$ e indefinitamente esteso nella dirczione delle $x$ positive, si prova che per lo spostamento verticale vale la relazione:

$$
\zeta=\frac{1}{1-v^{2} / c^{2}}\left\{F(t-x / v)-\frac{v}{e} F(t-x / c)\right\},
$$

dove $v$ è la velocità della perturbazione, $e$ quella delle onde libere del mare ed $F$ una funzione qualunque dei suoi argomenti. Nel caso del golfo di Trieste, il verificarsi dell'oscillazione libera, richiede che la propagazione della causa perturbante si effettui da Ovest ad Est, normalmente al fondo del golfo stesso ${ }^{2}$ ). 
Per quanto concerne la seconda condizione di risonanza, essa trova verificle molteplici. Ridotta alla sua più semplice espressione, la teoria può riassumersi nella segnente formula:

$$
\begin{gathered}
\eta=\frac{h M}{e^{2}\left(m^{2}-1\right)}\left[\cos k(x-v t)-\frac{m}{\sin 2 k l m}\{\sin k(l+v t) .\right. \\
\cdot \cos k m(x-l)+\sin k(l-v t) \cos k m(x+l)\}]
\end{gathered}
$$

dove $\eta$ rappresenta lo spostamento verticale, $c$ la velocità di propagazione di un'onda lunga, 9 la densitì, $v$ la velocità dell'azione perturbante, rappresentata da

$$
M_{0}=\mathrm{M} \cos h(x-v t),
$$

in eui $\mathbf{M}$ ̀̀ nu numero generalmente piccolo. The condizioni ai limiti sono rappresentate da spostamento orizzontale nullo per $x=$ 上 $l$, essendo $2 l$ la lunghezza del bacino chiuso. Con lievi varianti, la relazione vale anclıe per bacino aperto. $\mathbf{E}$ inoltre $h$ la profondità media del bacino e $m=v / e$.

In prima approssimazione, possiamo ritenere

$$
e=1 \cdot \bar{h}, \quad T_{i}=\frac{1 l}{21 / \bar{h}} \quad \text { per } i=1,2, \ldots
$$

Contrariamente all'apluarenza, in questo caso, per $v=c, \eta$ non tende all'infinito, mentre si ha risonanza quando $\sin 27 l m \rightarrow 0$. Se $s$ è un intero, si ha allora, posto $2 k l m=s \pi, \frac{T_{s}}{T_{s}}=\frac{s}{i}$, dove $T_{s}$ è il perioclo della forza perturbante (*).

Questa elementare teoria, mi ha consentito di spiegare l'insorgere delle onde interne del lago di Bracciano $\left({ }^{3}\right)$ e del lago di Garda $\left({ }^{4}\right)$, il formarsi delle oscilazioni libere (uninodali, binodali, ...) del lago di Caldonazzo $\left({ }^{5}\right)$, nonché le mareggiate della rada di Civitavecchia $\left({ }^{78}\right)$.

(*) Nel caso ili golf, baie, matri aperti è

$$
\begin{aligned}
& \eta=\frac{h . H}{e c^{2}\left(n t^{2}-1\right)} \mid \cos k(x-v l)-\frac{m \sin k(l-v i)}{\sin 2 k l m}\{\cos k m(x+l)- \\
& -\cos k m(x-l)\}] \text {, thove } \eta=0 \text { per } x=0 \text { (bocea tiel bacino iperto, di lun- }
\end{aligned}
$$
ghezza $l$ ). ì inoltre $\frac{T}{T_{z}}=\frac{8}{2 i-\hat{i}}$. 
Non v'ha dubbio che questa causa perturbante entra fra quelle che determinano il fenomeno delle acque alte nelle lagune venete; e ciò tutte le volte che l'interazione fra aria ed acqua è capace di eccitare le oscillazioni libere dell'alto Adriatico o dell'intero Adriatico.

Come spiegare allora l'allarmante ammentata frequenza delle acque alte nelle lagune venete $e$, in particolare, a Venezia?

Supposta immutata la morfologia dell'Adriatico, come fondo e come livello, bisognerebbe pensare ad un mutamento nelle condizioni meteorologiche, a cui detti fenomeni sono legati. Sebbene questa ipotesi, per quanto avremo modo di vedere più avanti, non sembri affatto azzardata, non spetta a me provarne o meno la validità; altri eventualmente potrì interloquire al riguardo, con maggiore cognizione di causa.

Na è davvero rimasta costante, negli ultimi decenni, la morfologia dell'Alto Adriatico?

\section{2. - Mutamentit MoRfolobici DELe'Alto ADRIATICo,}

A rigore, nessun bacino idrico, per quanto grandle, conserva inalterata la sua forma: variazioni di livello, avanzamenti di spiaggia per progressivo insabbiamento, retrocessioni di sponda per erosione, mntamento del fondo per apporti fluviali, ecc. sono agenti che tendono a modificare di continuo la forma di un bacino e il volume delle acque.

Tali mutamenti sono inapprezzabili nel tempo, nei confronti degli Oceani. Son sono però del tutto trascurabili con riferimento ai mari interni e possono verificarsi con grande rapidità nel caso di piccoli bacini chiusi.

Tra i mari interni, l'Adriatico - in particolare l'alto Adriatico è certamente fra $\mathrm{i}$ più soggetti a mutamenti clel genere.

î certo che, ancora nei primi secoli dell'era volgare, l'Adriatico si internava, molto più di adesso, nella pianura padana, occupando tutta. la linea da Ravenna ad Aquileia; e che, parechi secoli avanti Cristo, esso spaziava in gran parte del territorio, che comprende attualmente il Ferrarese, la bassa Padovana, il Polesine ed altre terre venete: Ravenna, porto tanto importante all'epoca romana, e Adria stessa - che diede il nome al mare che la bagnava - sollo oggi molto internate nella terra ferma.

Contemporaneamente, si verificava un progressivo abbassamento del sottosuolo dovuto sia a compattizzazione dello strato super- 
ficiale molle e melmoso, sia alla lenta flessione del fondo della fossa adrio-padana. Con ciò si spiega l'affossamento di cittì come Spina, Eraclea, Altino, ... semisommerse fra fango e canneti.

L'interrimento jrodotto dai fiumi e l'abbassamento del sottosnolo furono le cause principali che generarono, nei secoli, tante e così raflicali mutazioni noll'estuario veneto-padano $\left(^{6}\right)$.

Anche oggi, nel complesso, ì la terra che la vince sul mare; l'opera lenta di quelle forze, che hanno già interrato la jianurapadana, continua incessante: le sabbie, il fango, il limo trasportati dai fiumi fino alla foce e spinti in mare, ivi si espandono, per depositarsi infine sul fondo, colmandone le parti più basse e contribuendo alla formazione di uno strato, dolcemente declinante verso l'alto mare, strato che, lentamente, eleva il fondo stesso.

Anche a jrescindere dalle variazioni di spiaggia - accentuate al massimo in corrispondenza del Delta padano - , il fondo dell'alto Adriatico è quindi sottojosto a continui cambiamenti per l'apporto di torbida dei fiumi. P. es., l'Adige, a Boara Pisani (nei jressi della foce), presenta una media portata annuale fli torbida dell'ordine di 370.000 .000 tonn. [dedotta dalle caratteristiche torbiometriche medie anmue del jeriodo $1957-1963$ ( $\left.^{7}\right)$ ]. Se si considera che, nell'alto Adriatico confluiseono l'Isonzo, il T'agliamento, il Livenza, il Piave, il Brenta, l'Adige, il $P^{3} 0$, il Reno, ... - jer non citare che i fumi principali - ci si rende facilmente conto dell'enorme mole di materiale che incessantemente affiuisce in questo tratto di mare; e l'affiuenza assume sovente proporzioni grandiose, in corrispondenza delle piene, e dello straripamento dei fiumi jresso la foce, in particolare del Po.

Il trasporto del materiale di torbida interessa, indubbiamente nei suoi componenti più fini - tutto l'alto Adriatico.

Certo, una valutazione precisa dell'alterazione del fondo dell'alto Adriatico nel temjo, non è di facile realizzazione. Necessiterebbe dis jorre di attendibili carte batimetriche, relative a campagne di misura frequenti ed esaurienti. Purtroppo, questa condizione non è soddisfatta che in modesta misura.

Ad ogni moto, l'ordine di grandezza delle variazioni jiù cospictue può essere tratto dal confronto di carte batimetriche, relative a misure opportunamente scaglionate nel tempo. A questo proposito, la fig. 7 si riferisce al confronto dell'andamento del fondo, effettuato in tre direzioni diverse, valendomi di carte batimetriche del $1867-1873\left(^{8}\right)$ e flel 1896 (1928) $\left({ }^{9}\right)$. Si noter⿳亠 che, in tutti e tre i casi esaminati, la tendenza del fondo è stata decisamente verso il sollevamento. Questo vale anche 
per confronti in altre direzioni, che qui si ritiene superfuuo riportare (*). Naturalmente, i risultati sono solo indicativi, di grossolana approssimazione, sufficienti, comunque, a comprovare un progressivo, lento sollevamento del fondo dell'alto Adriatico, conseguente all'enorme quantità di materiale d'erosione che i fumi ivi sfocianti vi convogliano dalle $\Lambda$ lpi e dagli $\Lambda_{\text {Jjpennini. }}$

Torneremo più avanti su questi argomenti.

Ora ritengo utile fare aleuni richiami di Irlrodinamica.

\section{3. - SUtLE OSCHAAZIONI TIBERE E FORZATE DI UN BACINO SOGGETTO A MAREA.}

3.1. - Generalita. E noto che un bacino idrico, chiuso o aperto, puó assumere due tipi di oscillazioni: le oseillazioni projrie (o libere) e le oscillazioni forzate. Il periodo delle prime dipende dalla configurazione del bacino, mentre il periodo delle seconde coincide con quello della forza perturbante ed è quindi indipendente dalle caratteristiche geometriche del bacino.

Se $T$ è l'energia cinetica di un sistema meccanico - costituito da un numero discreto di junti materiali - avente un numero finito $n$ di grali di libertà, la cui situazione puó essere definita da $n$ parametri $q_{1}, q_{2}, \ldots q_{n}$, se $q_{r}^{\prime}=\frac{d q_{r}}{a i}$ e se $U$ rappresenta l'energia potenziale dovuta alle forze interne, le equazioni di Jagrange relative al moto del sistema si serivono

$$
\frac{d}{d t} \frac{d T}{d q_{r}{ }^{\prime}}-\frac{d T}{d q_{r}}+\frac{d U}{d q_{r}}=Q_{r} \quad(r=1,2, \ldots n)
$$

dove $Q$, rajpresentano le forze esterne.

I'estensione ai problemi delle maree si otterrà sostituendo degli integrali alle somme linite.

(*) Non si sono fatti confronti con più recenti batimetrie, sia perclıé queste sono state riportate sn carte a scala alquanto ridotta, sia perelé $i$ risultati sono raceolti in linec isolsate c non riportati per punti, come nolle determinazioni più antiche. I rilievi batimetrici, eseguiti recentemente per conto del C.N.R. nell'alto Adriatico $\left({ }^{10}\right)$, risulteranno preziosi per futuri rafironti. 
Le [1] valgono solo per movimenti assoluti. Atteso il moto di rotazione della Terra, ne consegue un moto relativo dei mari. Se $q_{0}\left({ }^{*}\right)$ flefinisce l'orientamento attrale del sistema nello spazio assoluto $\left(\frac{d f}{d q_{0}}=\frac{d U}{d q_{0}}=0\right)$, in mancanza di coppie esterne tendenti a far variare la rotazione della Terra $\left(Q_{0}=0\right)$, l'equazione di Jagrange relativa al parametro $q_{0}$ si riduce a $\frac{d}{d i} \frac{d T}{a \sigma_{a}{ }^{\prime}}=0$ e, quindi,

$$
\frac{a T}{d q_{0}}=p_{0}
$$

$p_{0}$ essenclo una costante. Fatto

$$
H=T-U-p_{\circ} q_{a}^{\prime}
$$

si trova che le equazioni di Jagrange divengono (11)

$$
\frac{d}{d t} \frac{d H}{d q_{r}^{\prime}}-\frac{d I I}{d q_{r}}-Q_{r} \quad(r=1,2, \ldots n)
$$

Tue equazioni di Lagrange conservano quindi la stessa forma, quando si considera l'equilibrio nel movimento relativo.

3.2. - Studio delle oseillazioni proprie. Quando le forze esterne sono nulle $\left(Q_{r}=0\right)$, l'equazione di Tugrange relativa ul parametro $q_{k}$ diviene

$$
\frac{d}{d \bar{l}} \frac{d I}{d q_{k}^{\prime}}-\frac{d I I}{d q_{k}}=0
$$

Si tratta fi $n$ equazioni lineari a coefficienti costanti.

Esprimiamo i diversi elementi di $I I$, in funzione delle cłratteristiche meceaniche del sistema. Avremo

$$
T=\sum \frac{m}{\underline{D}}\left(x^{\prime 2}+y^{\prime 2}+z^{\prime 2}\right)+q_{o}^{\prime} \sum m\left(x y^{\prime}-y x^{\prime}\right)+q o^{\prime 2} \sum \frac{m}{2}\left(x^{2}+y^{2}\right),
$$

(*) Supposti tre assi mobili invariabilmente legati alla Terra solida $\theta$ altresi tre assi fissi, gli assi $z$ coincidendo nei duc sistemi, allora $q_{0}$ rappresenterà I'angolo degli assi unobili con gli assi fissi; o le altre coordinate $q_{r}$ definiscono In posizione relativa del sistema rispetto argli assi mobili. 
dove $m$ rappresenta la massa di un punto materiale di coorlinate $x$, $y, z$ rispetto agli assi liberi e gli apici significano derivate rispetto al tempo. Se $T^{\prime}$ esprime la semiforza viva del sistema nel sno moto relativo, $M$ il momento di rotazione e $J$ il relativo momento d'inerzia, possiamo serivere

$$
T=T^{\prime}+q_{0}^{\prime} M+q o^{\prime 2} \frac{J}{2}
$$

E inoltre

$$
p_{0}=\frac{d T}{d q_{0}^{\prime}}=M+q_{0}^{\prime} J
$$

per cui

$$
H=T^{\prime}-U-\frac{1}{2 J}\left(p_{0}-M\right)^{2}
$$

Attesa la piccolezza delle coordinate e delle velocità, grazie alla quale possono essere traseurati tutti i termini superiori al 20 grado, ordinando rispetto alle $q^{\prime}$, avremo

$$
H_{2}=T^{\prime}-\frac{M^{2}}{2 J} ; H_{1}=\frac{p_{o} M}{J} ; H_{o}=-U-\frac{p_{o}^{2}}{2 J} .
$$

Per eui, mettendo in evidenza le diverse parti di $H$, le [3] divengono

$$
\frac{d}{d t} \frac{d H_{0}}{d q_{k}^{\prime}}, \frac{d}{d t} \frac{d H_{1}}{d q_{k}^{\prime}}-\frac{d H_{1}}{d q_{k}}-\frac{d H_{0}}{d q_{k}}=0 .
$$

Poiché $H_{2}$ è un polinomio di $2^{\circ}$ grado in $q^{\prime}, \frac{d H_{2}}{\vec{a} q_{f^{\prime}}{ }^{\prime}}$ sarì della for$\operatorname{ma} \frac{d H_{2}}{d q_{x_{i}^{\prime}}}=\sum_{j} a_{r k}{ }^{\prime \prime} a_{r}^{\prime}$, da cui

$$
a_{r k^{\prime \prime}}=\frac{d^{2} H_{2}}{d q_{r}^{\prime} d q_{k}^{\prime}}
$$

le ark essendo delle costanti.

Si prova (Poincaré, l.c., p. 12) che, essendo $\Pi_{1}$ un polinomio di primo grarlo in $q$ e in $q^{\prime}$, fatto

$$
a_{r k}^{\prime}=\frac{d^{2} H_{1}}{d q_{r} d q_{k}^{\prime}}-\frac{d^{2} H_{1}}{d q_{r}^{\prime} d q_{k}},
$$


ne segue

$$
\frac{d}{d t} \frac{d \Pi_{1}}{\pi q_{k}^{\prime}}-\frac{d H_{1}}{d q_{k}}=\sum a_{r}^{\prime} q_{r^{\prime}} .
$$

Mentre, essendo $H_{\circ}$ un polinomio di $2^{\circ}$ grado in $a$. fatto

$$
a_{r k}=-\frac{d^{2} H_{2}}{d q_{r} d q_{k},}
$$

consegue

$$
-\frac{d H_{0}}{d q_{k}}=\Sigma a_{r k} q_{r} .
$$

Per eui, le equazioni di Lagrange [4] prendono la forma

$$
\sum\left(a_{r k}{ }^{\prime \prime} q_{r}^{\prime \prime}+a_{r k} q_{r k}+a_{r k} q_{r}\right)=0 .
$$

Si tratta di $n$ equazioni lineari a coefficienti costanti, il cui sistema prò essere integrato ponendo

$$
q_{r}=a_{r} e^{\lambda t}
$$

dove $\lambda$ e $\alpha_{r}$ vanno determinate in modo che le equazioni vengano soddisfatte. Per sostituzione, queste ultime divengono allora

$$
\sum \alpha_{r}\left(a_{r k^{\prime}} \lambda^{2}+a_{r k^{\prime}} \lambda+a_{r k}\right)=0
$$

oppure, fatto

$$
\begin{gathered}
C_{r k}=a_{r k}{ }^{\prime \prime} \lambda^{2}+a_{r k}{ }^{\prime} \lambda+a_{r k k}, \\
\\
\sum a_{r} C_{r k}=0 .
\end{gathered}
$$

Si tratta ri $n$ equazioni lineari omogenee in $n+1$ incognite, $a_{n}$, $\alpha_{3}, \ldots \alpha_{n}, \lambda$. Il problema è possibile se il determinante di dette equazioni è nullo:

$$
\Delta(\lambda)=0
$$

D'altronde, si prova che $\Delta(\lambda)=\Delta(-\lambda)$. Pertanto, la [6] lia radici uguali a due a due e di segno contrario.

Nel caso di equilibrio stabile - che a noi più interessa -, queste radici (Poincaré, l.c., p, 1.4) sono puramemle immaginarie:

$$
\lambda=i \mu,
$$


essendo $\mu$ reale. Si hanno quindi $2 n$ soluzioni particolari, soddisfacenti alle equazioni di Jagrange e corrispondenti alle $2 n$ radici della [6]. Esse costituiscono le soluzioni proprie armoniche del sistema; funzioni periodiche del tempo, proporzionali ad un esponenziale immaginario.

Se

$$
\alpha_{r}=\varrho_{r} e^{i \omega_{r}},
$$

eiascuna delle oseillazioni proprie armoniche complesse del sistema, sarà data dagli $n$ valori

$$
q_{r}=\varrho_{r} e^{i\left(\mu t+\omega_{r}\right)}
$$

dei parametri $q$.

Poiché le equazioni differenziali sono lineari e a coefficienti reali, la parte reale e la parte imnaginaria delle soluzioni complesse sodilisferanno separatamente il problema. Sicclé, ad ogni soluzione complessa, corrisponderanno due soluzioni reali

$$
\begin{aligned}
& q_{r}=\varrho_{r} \cos \left(\mu t+\omega_{r}\right) \\
& q_{r}=\varrho_{r} \sin \left(\mu t+\omega_{r}\right) .
\end{aligned}
$$

Sono queste le oscillazioni proprie - libere - armoniche reali del sistema. Combinando le $2 n$ soluzioni particolari delle equazioni differenziali, si ottiene la soluzione generale del problema.

Il periodo di un'oscillazione libera, corrispondente al valore di $\mu$, è dato da $\frac{2 \pi}{\mu}=\frac{2 \pi}{\lambda} i$. L'equazione in $\lambda$ definisec quindi i periodi delle oscillazioni libere.

Determiniamo $\varrho_{r}$ e $\omega_{r}$, cioè $\alpha_{r}$.

Se indicliamo con $D_{r k}$ i minori del determinante $A(\lambda)$, avremo

$$
\Delta=\Sigma C_{r k} D_{r k} \text {. }
$$

Dalla teoria delle equazioni lineari omogence, consegue

$$
\frac{\alpha_{1}}{D_{1 k}}=\frac{\alpha_{2}}{D_{2 k}} \ldots \ldots=\frac{\alpha_{n}}{D_{n k}} .
$$

I minori sono quantità complesse interamente determinate, una volta noto $\lambda$. Ciò permette di determinare $\alpha_{r}$. 
3.3. - Studio delle oseillazioni forzate. Quando agiseono forze esterne non nulle, il termine $Q$, dell'equaione di Jagrange, relativo al parametro $q r$, sarì della forma

$$
\vartheta_{r}=\sum K_{r h} c^{\lambda_{H} t}
$$

i futtori esponenziali essento funzioni armoniche del tompo.

Consisteriamo le componenti amplesse della forza perturbatrice. Ciascuna di essa dara luofo ad un'oseillazione forzatu isocrona dello stesso poriodo; e quando tutte le componenti agiranno all'wuisono, l'oscillazione risultente, per il principio della sovapposizione dei piccoli movimenti, sara la somma delle oscillezioni dovute a eiascuna desse.

Esaminiamone pertanto una sola. Poniamo

$$
Q_{r}=K_{r} e^{\lambda t} \text {. }
$$

A questa forza perturbante corrisponterà un'oscillazione forzata

$$
q_{r}=\varepsilon_{r} e^{\lambda t} \text {. }
$$

In tal caso, le equazioni di Jagrange divengono

$$
\frac{d}{d t} \frac{d H}{d q_{i}^{\prime}}-\frac{d H}{d q_{t}}=\pi_{*} e^{\lambda l} \quad(k=\text { i. } 2 . \ldots n)
$$

Distinguendo le tre parti di $H$ gia considerate nel precodente paragrafo, le [8] divengono

$$
\sum\left(a_{r k}^{\prime \prime} q_{r}^{\prime \prime}+a_{r k}^{\prime} q_{r}^{\prime}+a_{r k} q_{r}\right)=K_{k} e^{\lambda t},
$$

con $k$ ed $r$ varianti da 1 ad $n$.

Per la [7] e ricordando la [5], av'remo il gruppo di equazioni

$$
\sum \varepsilon_{r} C_{r k}=K_{k}
$$

Poiché $\lambda$ è assegnato, le $C_{r k}$ sono costanti note.

Ciascuna thi queste equazioni comprente le $n$ incognite $\varepsilon_{r}$; e le equazioni sono $n$. Sono, come le [5], equazioni di primo grado; non piì omogenee, però, avento un secondo membro. Verranno pertanto risolte col metodo di Trimer. Ogni soluzione avrà a denominatoro il determinante dei coefficienti $C_{r k}$, delle incognite, mentre a numeratore 
figurerà lo stesso determinante, in eui verrì sostituito $C_{r 1}, C_{r g} \ldots C_{r m}$, ris]ettivamente con $K_{1}, K_{2}, \ldots K_{n}$ [ciò̀ $\left.\Sigma K_{n} D_{r h}(\lambda)\right]$.

Una soluzione particolare del problema delle oscillazioni forzate ì quindi formita dagli $n$ valori

$$
\varepsilon_{r}=\frac{\sum K_{n} D_{r h}(\lambda)}{\Delta(\lambda)} .
$$

3.4. - Confronto fra le oscillazioni for ate e le oscillazioni libere. Ja grandezza $\varepsilon_{r}$ ì una funzione razionale che puo essere scomposta in elementi semplici.

Indicando con $\lambda$, una delle $2 n$ radiei dell'equazione $A(\lambda)=0$, abbiamo visto che l'oseillazione propria conispondente è data dai valori dei parametri

$$
q r j=\alpha_{r j} \cdot e^{\lambda_{j} t}
$$

eon $j$ variabile da 1 a $2 n$ e $r$ variabile da 1 a $n$.

Questi valori di $q$ sono soluzioni delle equazioni dillerenziali omogenee, laddove $q_{r}=\varepsilon_{r} e^{j t}$ ¿̀ un soluzione particolare delle stesse equazioni con secondo membro.

Il problema delle oseillazioni forzate sarà quindi risolto, aggiungendo ala soluzione particolare una soluzione qualunque delle equazioni omogenee, cioè una soluzione propria. Le radici del denominatore, $\lambda=\lambda_{s}$, sono quelle cle definiscono i periodi di oscillazione libera del sistema: ad ognuna di tali radici corrisponderà un termine $\frac{\sum K_{H} D_{r h}\left(\lambda_{s}\right)}{\left(\lambda_{-}-\lambda_{f}\right) \Delta^{\prime}\left(\lambda_{s}\right)}$, $(h=1,2, \ldots n)$. Sommando rispet to a $j,(j=1,2, \ldots 2 n)$, avremo pertanto

$$
\varepsilon_{r}=\sum \frac{\sum K_{H} D_{r_{A}}\left(\lambda_{j}\right)}{\left(\lambda_{H}-\lambda_{j}\right) \Delta^{\prime}\left(\lambda_{j}\right)} .
$$

Le soluzioni immaginarie coniugate essendo $s_{r j}=\beta_{r j} e^{\lambda, t}$, si dimostra (Poincaré, 1.c., pp. 15-16, 20) ehe il rapporto $\frac{D_{r h}\left(\lambda_{s}\right)}{\alpha_{r s} \beta_{h s}}$ ̀े indipendente da $r$ e da $h$; per eui possiamo scrivere

$$
\frac{D_{r h}\left(\lambda_{j}\right)}{a_{r j} p_{h f}}=\mu_{I} \Delta^{\prime}\left(\lambda_{j}\right) ;
$$

ne segue

$$
\varepsilon_{r}=\sum \sum \frac{K_{h} \mu_{j j} \alpha_{r j} \beta_{h j}}{\lambda-\lambda_{j}},
$$


da eui seguono le espressioni dei parametri $q_{r}$. Serivendo $q_{r^{\circ}}$ per indicare che si tratta di una soluzione particolare telle equazioni non omogenee, avremo

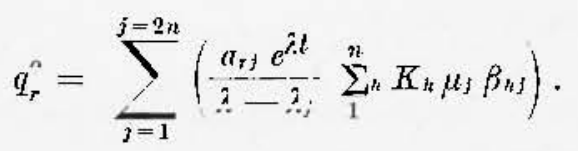

$\lambda$ e $K_{n}$ sono costanti date, in quanto si riferiscono alla forza perturbatrice conosciuta; $\lambda_{j}, \mu_{s}, a_{r}$ e $\beta_{h}$, sono pure costanti, relative all'oscillazione libera, il cui periodo è definito dallı radice $\lambda_{s}$ dell'equazione [6].

Si flimostra (Poincaré, l.e., p. 26) che $2 \mu_{k}=-\lambda_{k}$; per cui, fatto

$$
T_{0}=\sum K_{h} \beta_{h y},
$$

la [9] può scriversi

$$
q_{r}^{0}=-\frac{1}{2} \sum T_{o} \alpha_{r j} \frac{\lambda_{1}}{\lambda-\hat{\lambda}_{1}} e^{\lambda t}
$$

A meno di un coefficiente, the non dipende dis $j$, il termine generale di $q_{r}{ }^{\circ}$ sarà $\alpha_{r} e^{2 .}$. Ogni termine corrisponderà al un'oscillazione forzata armonica. Confrontanclo questa oscillazione con l'oseillazione libera corrispondente, si verte che $\alpha_{r}$ è la stessa per entrambe le oscillazioni, ma che il coefficiente esponenzinle è $e^{\lambda t}$ per la prima e $e^{\lambda \cdot t}$ per la seconda.

Ogni oscillazione forzata lia dunque, nei suoi diversi punti, le stesse differenze di fase doll'oscillazione propria armonica corrispondente e un'ampiezza proporzionale, ma il suo periodo ne differisce, essendo quello della forza perturbatrice.

Va qui sotitolineato un fatto d'estremo interesse. Supponiamo che 7. sia molto vicino ad uno dei valori $\lambda_{1}$, relativi alle oscillazioni libere.

Allora, il termine corrispondente nell espressione $d i$ q aiverva preponderante, e l'oscillazione forzala ossercata differira molto poco nel periodo, nel rapporto delle ampiezze $e$ nelle differenze di fase in diversi punti di una delle oscillazioni libere armoniche del sistema: e questa tendenza a coincidere è accompagnata da un'esaltazione del moto risultante. E' questo il caso della risonanza: se ne constata l'effettuazione in certi bacini marittimi, dove si manifestano delle maree considerevoli (baia di Fundy, ece.), prossime al una oscillazione armonica propria dei bacini stessi. 
Fino a che punto questo fenomeno interessa l'alto Adriatico? E, se ivi esiste anche solo in parte, qual'è la sua attuale tendenza? Le variazioni morfologiche contribuiscono ad aecentuarne gli efletti?

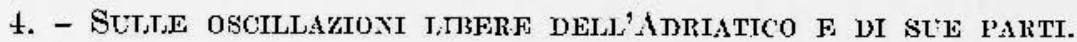

Le maree dell'Adriatico formarono oggetto di osservazione e di studio già da parecchi secoli. Giuseppe Toaldo ne tratta diffusamente in un suo lavoro del $1781{ }^{\left({ }^{2}\right)}$; in modo specifico nell'srticolo VIII: "Digressione sulla marea del Golfo Adriatico m, dove distingue la marea diurna, la "mestrua " (mensile) e l'unnua. Ma le ricerche più accurate si hanno verso la fine del secolo scorso e ayli inizi dell'attuale (13-1p).

Spetta a von Kesslitz l'aver provato - per la prima volta l'esistenza di un'oscillazione propria dell'Adriatico, il cui periodo valutò in $23^{\mathrm{n}}$ circa. Egli ritenne tale oscillazione l'uninodale dell'intero $\mathrm{Adria}$ tieo, con l'uninolo dove l'Adriatico si unisce al Mediterraneo (come effettivamente fu dimostrato da von Sterneek). Inche Defant $\left({ }^{20}\right)$ accettò le conclusioni di von Kesslitz e calcolò per il periodo dall'Adriatico, considerato come golfo del Mediterranes, il valore di $22 \mathrm{2n}, 1$, non lontano quindi da quello clee von Kesslitz trasse dalle osservazioni.

Yon Kesslitz aveva pure osservato monda stazimaria del periodo di $12^{\mathrm{h}}$ ca. Tale oscillazione (prossima alla marea semidiuma) fu all'inizio diversumente interpretata. Defant la ritenne come fondamentale dell'Adriatico, considerato come lago - e quindi con due ventri agli estreni Nord e Sud (Canale d'Otrunto). Altri l'attribuirono all'oseillazione della sola conea allriatica settentrionale aperta, per la quale von Sterneck indicava un periodo di $12^{\mathrm{h}}$ e Oddone di $\left.11^{\mathrm{h}}, 5{ }^{(21}\right)$.

Come nascono le oscillazioni libere nell'Ailriatico?

L'influenza delle variazioni di pressione atmosferica in bacinj cliusi, non si limita - come del resto si è già visto - all'azione statica; molto spesso essa induce influssi dinamici, dando luogo a dislivelli talora notevoli. Anche il vento agisee sul livello del mare. Nell'Adriatico, com's noto, lo scirocco tencle a spingere l'acqua verso le coste settentrionali, mentre la bora, soffiando da ENE provoca l'efietto opposto.

Franceseo Vercelli - che ebbe ad occuparsi ripetutamente dell'effetto delle perturbazioni atmosferiche snl livello del mare, - in uno studio del 1922 (22), mette in exilenza, nel diagramma esteso ad un anno di registrazioni del mureografo di Trieste, ondulazioni periodiche 
con periodo di circa 11 ore ed altere con periodo di circa 22 ore, astalogamente a (flanto aveva fato von kesslitz per i porti rli lobla (18) e di lagusa (19). Nello stesso lavoro, applicando alla curvo registrata il suo metexlo rli analisi periokale, Vereedli ottiene per le due oseillazioni accomate i prisoli di 22,2 ore e 11 are cirea, rispetivamente. I valori

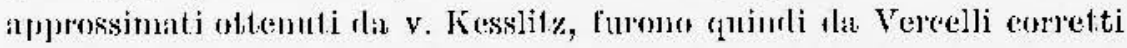
di cirea um'ura (in meno).

Per quanto riguarala l'unimorlale, un tentativo fatto da Odalone (a) un piceolo moklello dell'Arlriatico, lat portato a valori forke eecessivi ("').

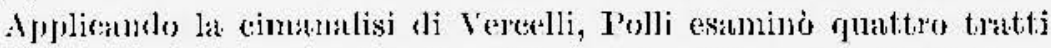
di curva maseogralies, regristrati a Trieste rispettivamente il 29 Genmaio-2 Febbraio [918, il 3-6 Ginguo 19-48, il 18-22 Ottobre 19-10 e il 15-17 $\mathrm{A}$ prile 1950 (23). Nai casi esamimati, Polli ottiene i valori di 21,1 $( \pm 0,7)$ ore; 21,$2 ; 21,5 ; 21,0)$ per l'uninolale alell'intero maro; 012,2 ore

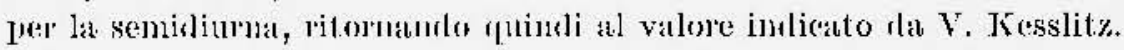

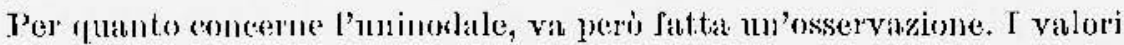
riportati somo in realtis delle modio. Dalla fig. 1 del lavoro ali I'olli (l.c., p. 70 ), la prims onda completan risulta avere un periodo di $200^{\mathrm{t}}, 5$

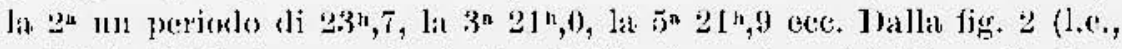

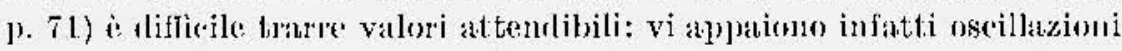

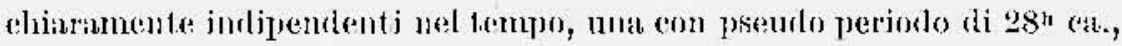

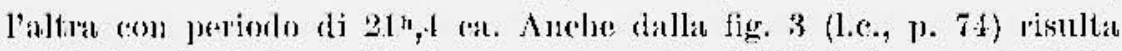

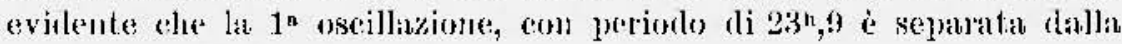
stecesssiva, di piecola ampiezza. Ritengo pertanto solo indicativa la

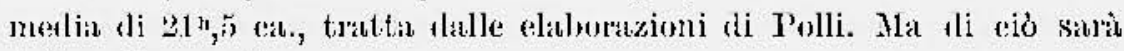
detto piil avanti.

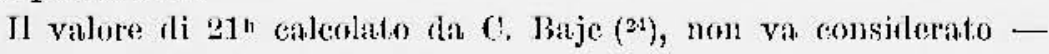
come del resto riconosece lo stesso $A$ - molto preciko, essemilo stato derlotito su sclematizzazioni troppos spinte, alle quali sluggono le preponderanti ronseguenzo dolla forma dol bacino sul periodo delle sue asseillazioni libero.

Solo un metorlo difrerenziale, ehe tenga conto delle continne variazioni della forma dol bacino, pui conture ad anjossimazioni aceet-

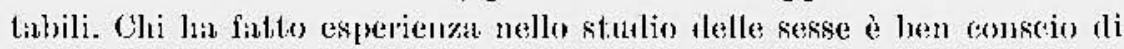
questa verità. Porlunto, a mio parere, i valori più attendibili yer $i$

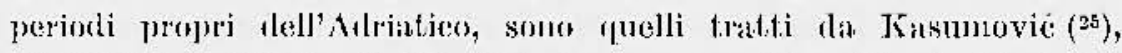
estendendo il netodo di Glorytal ad una quidentina di sezioni trasversali, che l'hamo condento and num curva nomale analoga a quella da 


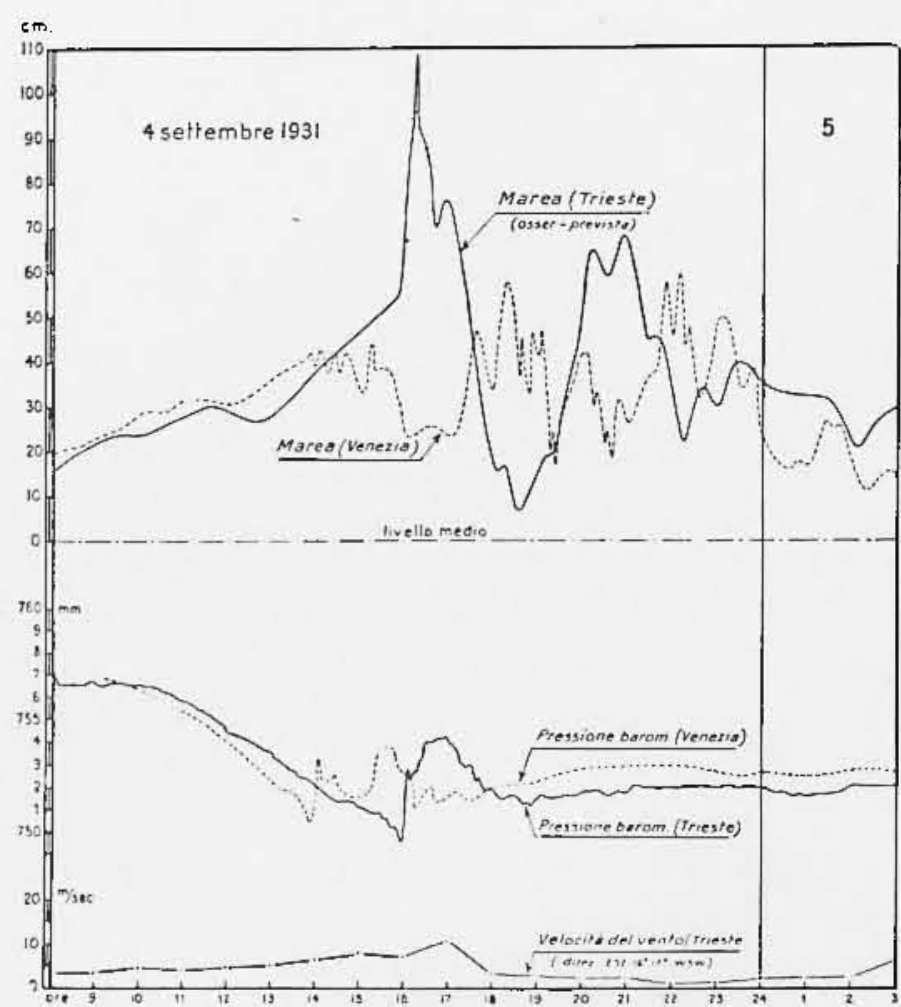

Fig. 1 - Interazione fra atmosfera ed idrosfera a Tricste, per propagazione di disturbi micrebarici verso la hase ilel golfo di Tricste, eon velocità dell'ordine di quella delle onde libere del mare sottostante $(2,5)$ : oltre alla sessa del golfo di Trieste (o Venezia), eon periodo di $3^{1 / 45^{\mathrm{m}}}$ ca, l'interazione provoca inizialtuente un balzo th fivello di $52 \mathrm{~cm}$ su breve periodo, in corrispondenza di dinamico pari a ca. 87 volte quello statico.

Fig. 1 - Interaction between atmosphere and hydrosplere in Trieste, due to the propagation of microbarie perturbations towarl the Gulf of Trieste, at a velocity in the oriler of that of the free waves of the underlying sea $(2,5)$, the interaction gives rise not only to the seiehe of the Gulf of Trieste (and Venice), with periods of about 3 hours and 45 minutes, but initially also to a beriod serturbtion of only $1 / 2$ Torr: therefore with a do microbaric equal to 87 times the static effect.

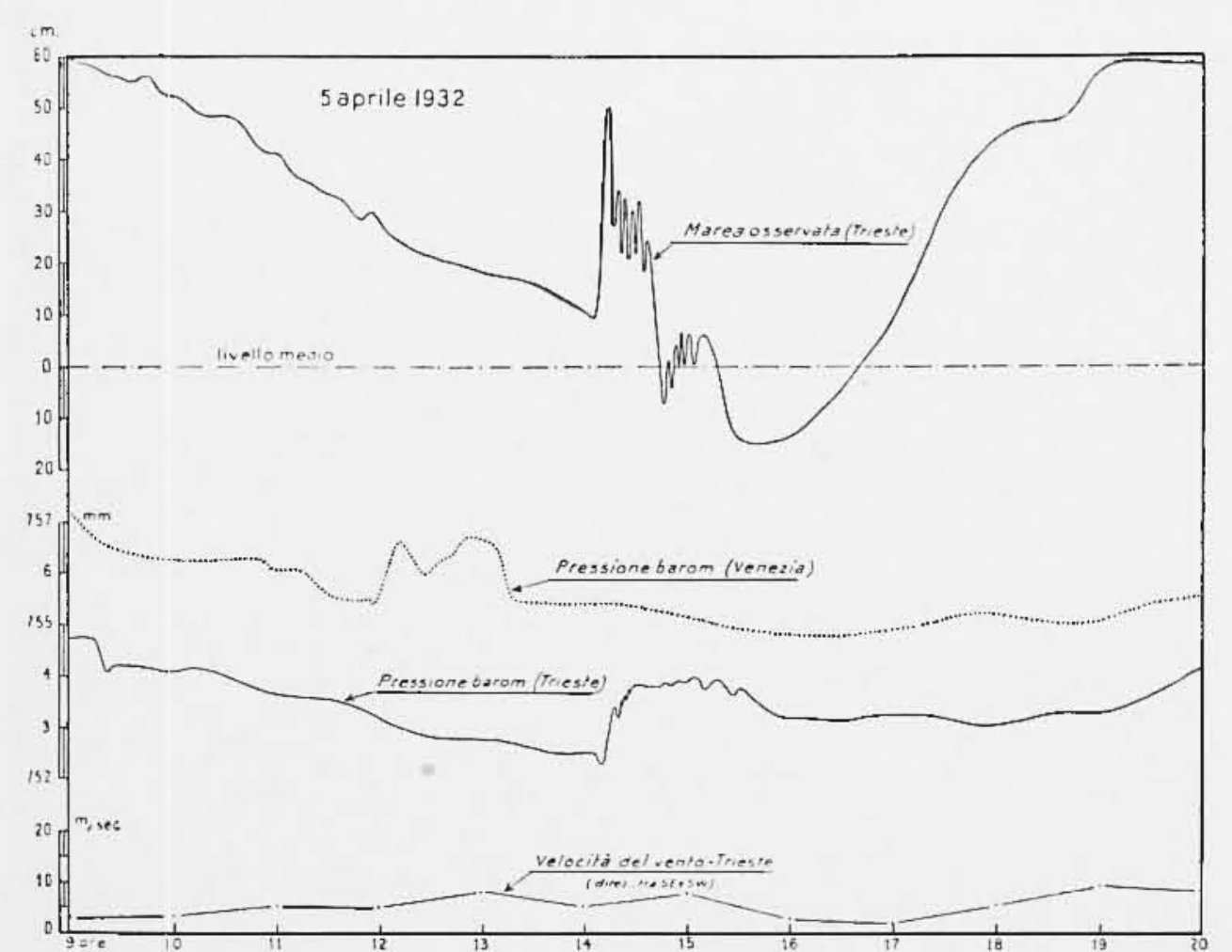

Fig. 2 - Altro chiarissimo escmpio di interazione fra atmosfcra ell inrosfera nel yolfo di Trieste particolarmente pronumciato nei brevi periodi. Il disturbo microbarico iniziale di circa $1 / 5$ di Torr, provoca um balzo nell acqua di ea. $40 \mathrm{~cm}$; eioè, l'effietto dinamico è eirca 150 volte quello statico. Fig. 2 - Another clear evidenec of interaction between atmosphere and The initial microbarie perturbance of about 1/5 Torr eauses the water to bounl some 40 crms; in other terms, the dynamic effect is about 150 times as strong as the static effect.

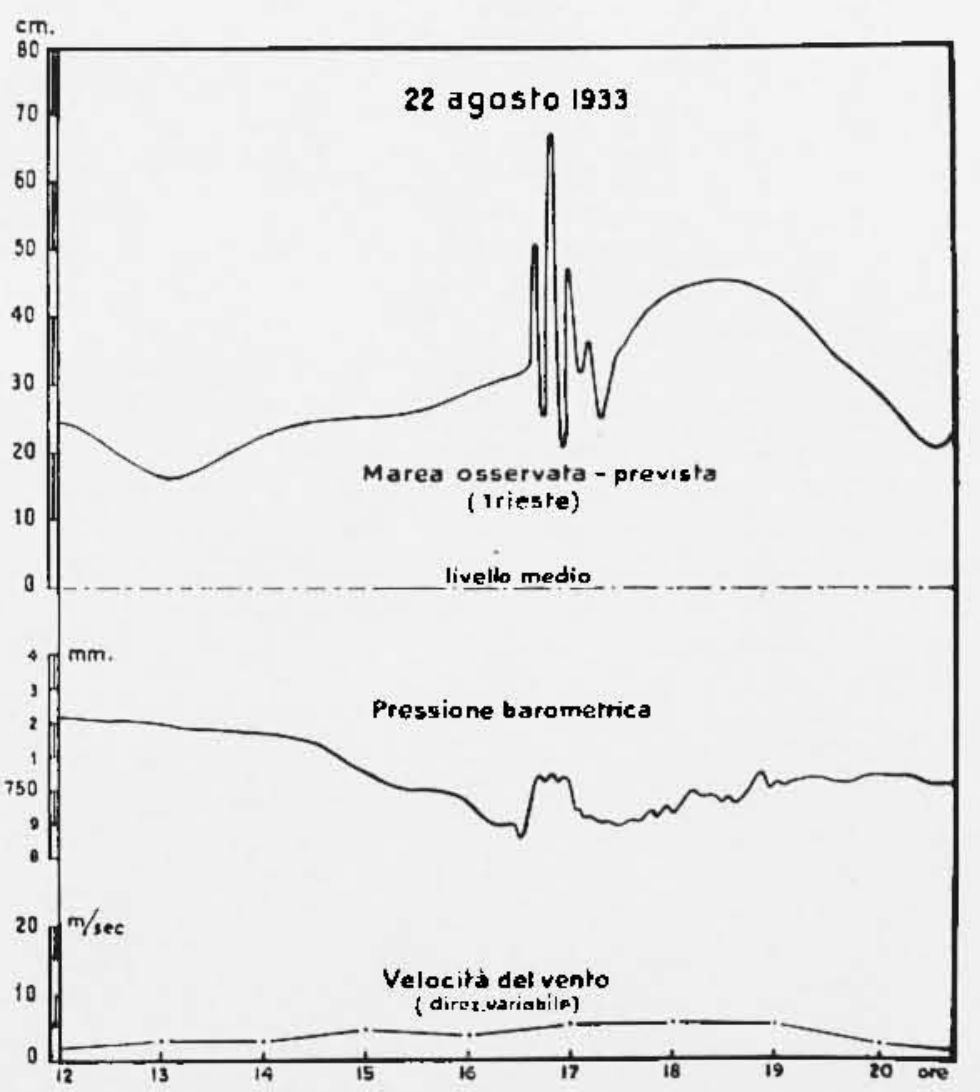

Fir. 3 - Anche in questo esempio, l'cffetto dinamico sni brevi perioli $\partial$ centinaia di volte quillo statico (v. figg. 1 o 2 )

Fig. 3 - In this example, too, the drnamic effect on short periods is hundrels of times as strong as the statie effect. (sce fig. 1 . and 2) 
me, a suo tempo, ottenuta per il lago di Levico; e quindi, allo stesso sistema di formule operative $\left.{ }^{25}\right)$. La fondamentale del mare Adriatico, come golfo aperto sul Ineliterraneo, risulta così avere il valore di $22^{h}, 11$ (metodo Clirystal) e $23^{\mathrm{h}}, 16$ (metodo Gol(lberg) - che si avvicina al valore di $23^{n}, 34$ calcolato da Defant - La media delle osservazioni, portano Kasumovic al valore di $22^{\mathrm{h}}, \overline{\mathrm{T}} \pm 0^{\mathrm{h}}, 8$, in buon accordo con $\mathrm{i}$ valori calcolati.

Per l'oseillazione propria di circa 12 ore, Kasumovic ottiene con il metodo di Chrystal, applicato al tratto di mare fra le coste venete e l'allineamento Gargano-Pelagosa-Lagosta-Curzola, considerato come lago -, il valore di $11^{\text {h }}, 34$; mentre la media delle osservazioni lo conducono a $11^{11}, 76 \pm 0,3$. L'ipotesi dell' $A$. ì suflragata dalle registrazioni mareografiche, che mostrano ventri in opposizione a Venezia e a Vieste, con linea norlale fra Ancona e l'isola Pago. Kasumovic giunge a questa conclusione, dopo aver provato erronea l'ipotesi di Defant, che faceva dell'oscillazione di 12 ore circa l'uninodale dell'intero Arlriatico, oscillante come lago.

Na torniamo, per un momento, sull'oscillazione propria, uninodale dell'Arriatico. Cone ho gì detto, a mio parere il suo periodo è quello calcolato da Kasumovic, di 22 ore almeno. Se essa è - come ì stato provato - la fondamentale dell'Adriatico, come golfo aperto sul Meditemaneo centrale, le leggi dell'Idrodinamica garantiscono la possibilitì del suo realizzarsi, in quanto la sua linen nodale si affaccia sopra un bacino eapace di oscillazioni proprie di più lungo periodo. Che questa sia la sua origine, è del resto provato anche dalle registrazioni, dove le onde si presentano con carnttere autonomo: a volte intervallate da lunghezza d'onda pressoché uguali (Vercelli, l.c., Tav. IV), a volte parzialmente sovrapposte (Polli, l.e., p. 70), talorn separate da intervalli più o meno lunghi (Polli, l.e., p. 71 ), o addirittura isolate (Polli, l.e., p. Tt). E ciò si spiega, tenendo presente che queste onde sono oscillazioni di un bacino, aperto sopra altro bacino molto pì̀ esteso. In tal modo, quando la causa perturbante agisce, l'oscillazione tormando dalla base del golfo, prosegue oltre il noto come onda progressiva, esaurentosi quindi in un periodo. L'insorgere di una nuova oscillazione, presuppone la continuità della causa origine, o la sua rijoresa, che può essere anticipata o posticipata su un intero periodo (27.a1). Qualsiasi metorlo di analisi periodale, ricliede la contimuita del treno d'onde investigato; mancando questa, i dati vengono forzatamente mediati, con " stiramenti" e "contrazioni " inevitabili, cle portano a risultati, spesso 
diseosti dalla renlti. I'er tali motivi, ritengo fiì attendibile, come modiat relle osservazioni, il valore di $22^{n,},-1-0 ", 8$, derlotto da Kasumovic con altri metodi.

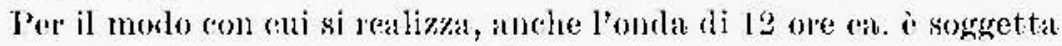
a rapide atfemazioni al ressare della causa prenturbatrice, sia pure in minor misum di quella di 22 ore ca.

Anche parti fin limitate dell'Arliatico sono ansaci di notevoli oscillazioni proprie (figg. I-6). ('osi, quando una perturbazione atmosferion si propaga da Ovest arl Esi attraverso l'alto Arluation, il golfo di Trieste contra in oscillazione libera, sul ritmo di $3^{\text {h}}, 5$ eiren, come iे stato da me povalo ne] 1\$37. Quando l'energin della forza jerturbante è sufliciente, la sessad del golfo di Trieste si tramuta nelloscillazione gropria del golfo di Venezia, come oscillazione trasversale rell'alfo Adriatico (2). Nella grandi masifestazioni meleofologiche, quesia è sempre presente. Poiche il primo massimo viene ragginnto a Trieste, 3,5-4 ore più tardi alla marea di Veneziat verra a sovmeporsi un incremento vautabile fra i 15 o i 40 am. Si vode in cio la possibilita di una preziosa - sia pur parmiale - previsione rli acpua alta per Venezia (figgr. 1, 4-6).

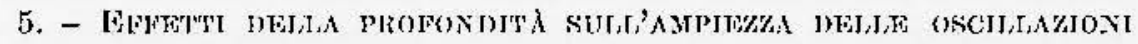
HIBWRT; O WORZATE DI CY BAOLO.

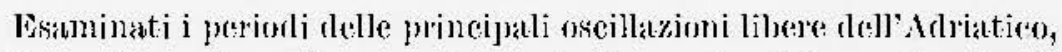
corchiamo di procisare il comportamento di queste ultime nel golfo rli

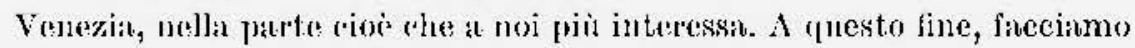
aleuni yeluiami di ioluorlinamien.

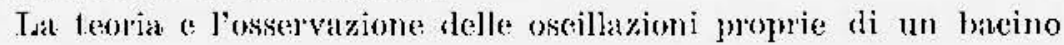
idlico provano che, a parità di alloe condizioni, l'ampiezan delle oscillazioni à sempre magriore ralla parte carat terizzata da minor profonditi e clue il divario aumenta con l'aumentase della differenza di profonditì ai rlue estremi (in easo di bacino eliuso). Per ess., per questo motivo, l'uninorlale del lago di ('akdonazzo, allestremo Nom (S. Cristoforo), la ampiezza ropejia di quella che si osserva a Sud (Lido di Caldonazzo) (:0.5), l'uninodate rle] lago rli Jevioo a Norrl is (puadrupla di quella che si rearistra a Sud $\left({ }^{2 n, 30}\right)$; jear il lago di Albano, sempre per lo stesso motivo, si osserva ad lest un'ampieza ciron quintupla di quella re]ativa all'estremo Ovest, dove il lago presenta nua notevole profondità ( $\left.{ }^{31}\right)$. J'elietto assume le più vistose manifestazioni presso i bacini artificiali, 


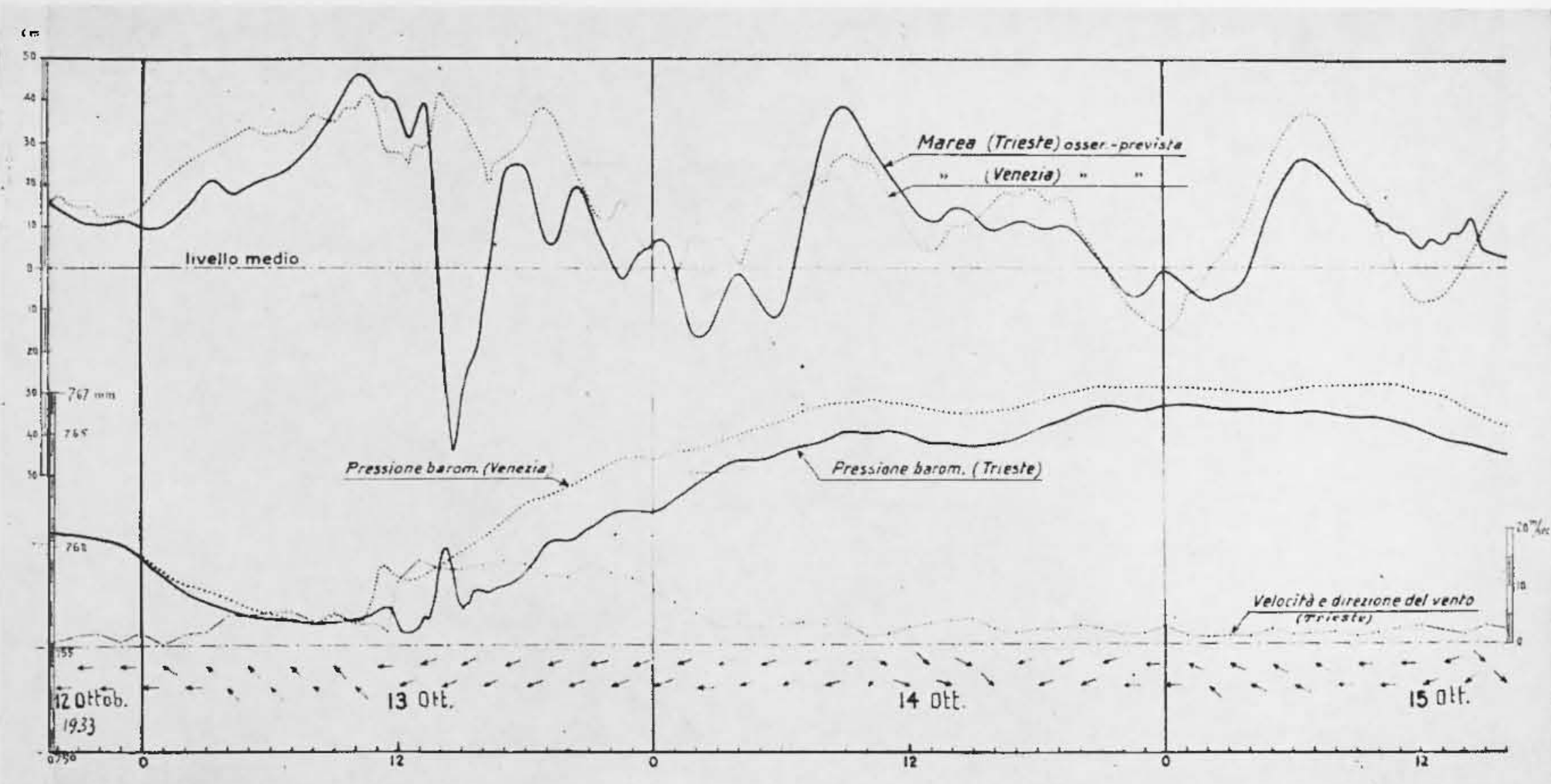

Fig. 4 - In questo caso, l'interazione fra atmosfera ed idrosfera si è manifestata sui lunghi periodi, destando le sesse del grolfo di Venezía (trasversali) e quella uninodale dell'intero Adriatico e dell'alto e medio Adriatico. L'iniziale nucleo positiso della pressione, dell'orline di 3 Torr, ha provocato un abbassamento del livello del mare a Trieste pari a $83 \mathrm{~cm}$, con un effetto dinamico più di 20 volte quello statico.

Fig. 4 - In this case the interaction between atmosphere and hydrosphere beeame apparent on the long periods by arousing the seiches of the Gulf of Venice (transversal) and the uninodal oscillation of the entire Adriatic sea and of the high and medium scetors of it. The initial positive pressure nueleus in the order of 3 Torr brought about a reduction of the sea level at Trieste of about $83 \mathrm{cms}$, with a dynamic cffect of over 20 times as strong as the static one.

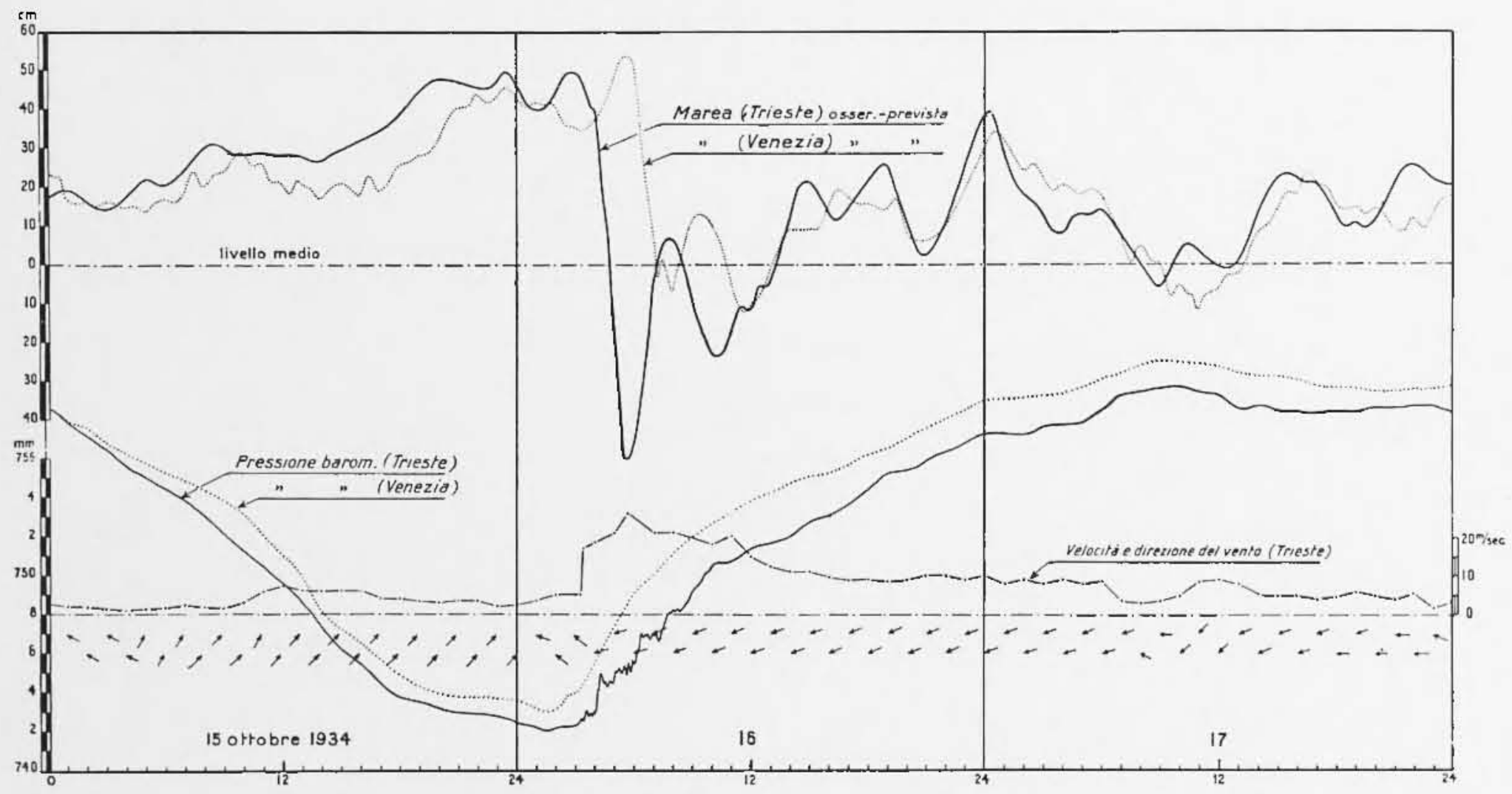

Fig. 5 - Analogo a quello della fig. 4. I'effetto dinamico iniziale è oltre 30 volte quello statieo.

Fig. 5 - Analogous to fig. 4. The initial dynamic effect is over 30 times as strong as the static effect.

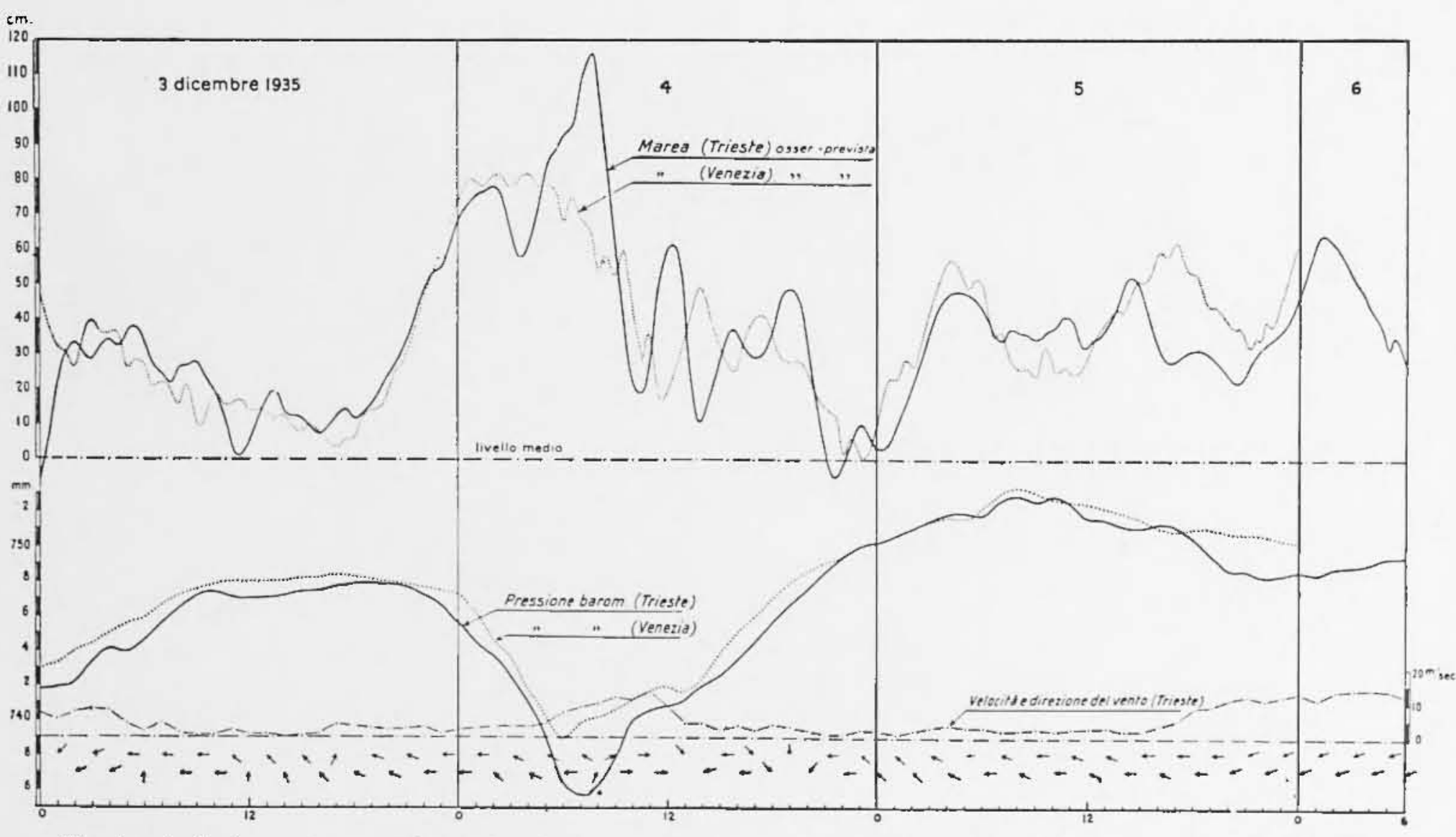

Fig. 6 - Anche in questo caso, l'interazione è stata particolarmente at tiva sui lunghi periodi, provocando oscillazioni libere del golfo di Trieste (Venezia), dell'intero Adriatico e della sua parte centro-settentrionale.

Fig. 6 - In this case, too, the interaction showed to be particularly active on the long periods; it brought about free oscilla tions of the Gulf of Trieste (Venice), of the entire Adriatic sea and of its central and northern sectors. 


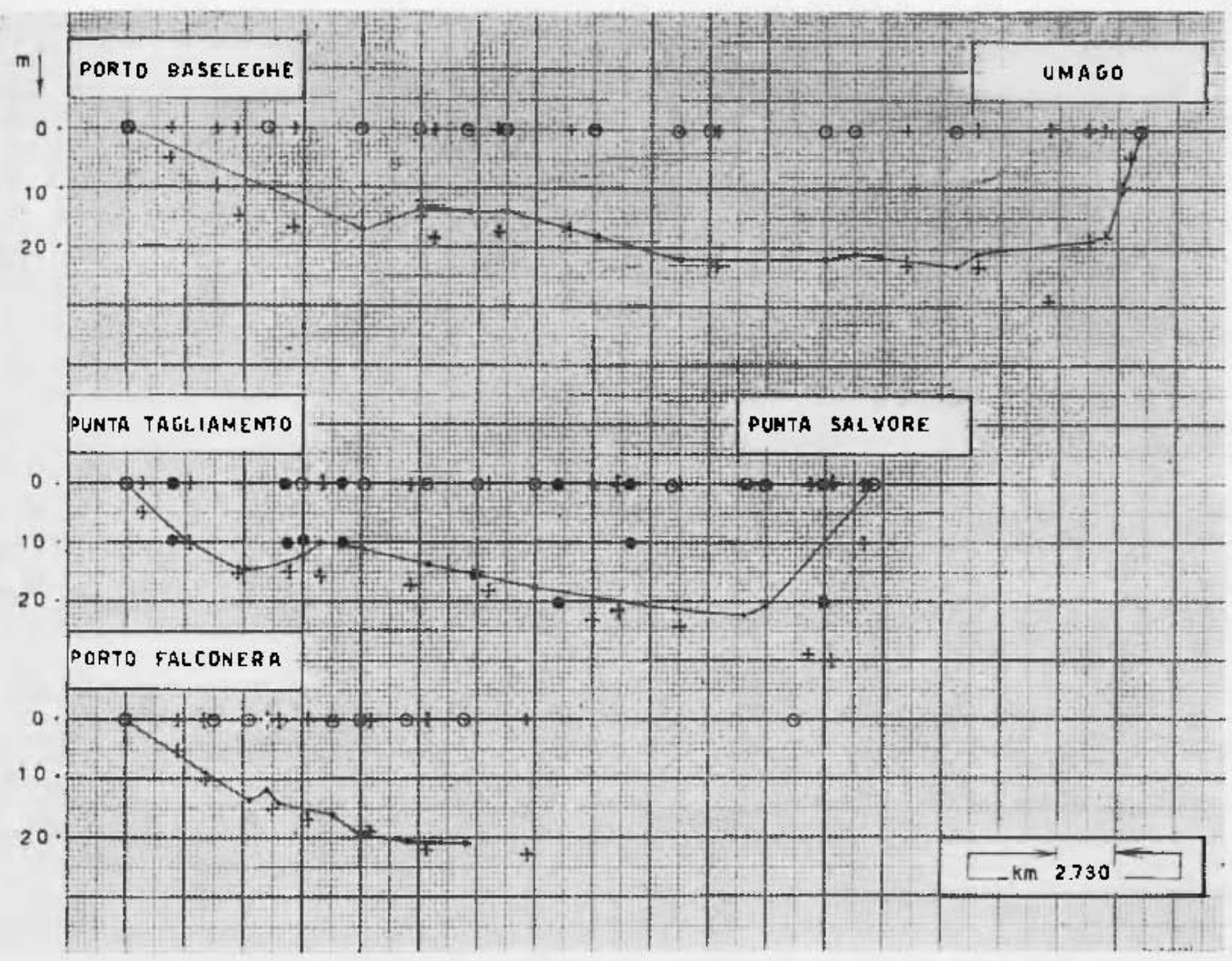

Fig. 7 - Sezioni dell'Alliatien fra Porto Baselegle c Umago, P'unta Tagliamento o Punta Salvotes, e Potto Falconera velso il punto $45^{\circ} \mathrm{N}, 13^{\circ} \mathrm{E}$, con andaucnto del fundo tratto ta carle hatimetriche del 1867 (crocelte separate), del 1896 (tratto continuo) c tel 1959 (cerchietti pieni).

Fig. 7 - Sections of the Aclriatic Sea between Porto Baseleghe and l'mago, l'unta Tagliamento and P'unta Salvure, and Porto laleoneta laward the point $45^{\circ} \mathrm{N}, 13^{\circ} \mathrm{L}$, showing the development, of the sea butifour taken from batlyunetric majs of 1865 (crossed line), of 1896 (full line) and of 1959 (dotted line).

formati dallo slauramento di valli alpine, fove le oscillazioni libere fondamentali dal lato della diga sono sempre notevolmente inferiori, in ampiezza, di quelle che si osservano nell'estremo più a monte.

Consideriamo il caso ilell'Ndriatico. In via approssimativa, questo mare può considerarsi formato da due conche consecutive: la settentrionale poco profonda (salvo la poro estesa fossa meso-arliatica), la 
meridionale con profondità superiore ai 1000 metri (figg. 8-9). La linea di divisione di queste due conche, puó considerarsi la congiungente Vieste-Ragusa, nei pressi della quale l'Adriatico scende, quasi bruscamente, dai 200 ai $1200 \mathrm{~m}$ (fig. 8).

Ciò premesso, consideriamo le oscillazioni libere di un bacino costituito da due conclıe sifiatte. Sclıematizzando il problema, immaginiamo un canale, limitato da due pareti verticali: $x=-b, x=+b^{\prime}$ e presentante una soglia in corrisjondenzil di $x=0$. Siano $h$ e $h^{\prime}$, rispettivamente, le profondità nei due scompartimenti (fig. 10), profondità ritenute molto piccole, nei confronti delle lunghezze d'onda.

Occorre trovare una funzione $\varphi$ di $x, y$ che soddisfi alla condizione (Poincaré, l.c., ]). 90):

$$
\frac{d^{2} \varphi}{d t^{2}}=g h \Delta_{2} \varphi
$$

essendo $\Delta_{2}$ il laplaciano di $\varphi$.

Poiché $\varphi$ è sempre proporzionale a $e^{\lambda t}$, essendo $\lambda$ espressione della pulsazione d'onda, nel caso delle oscillazioni libere possiamo scrivere

$$
\lambda^{2} \varphi=g \lambda_{2} \varphi
$$

Con riferimento alla parte profonda del bacino, poniamo

$$
\varphi=A e^{2 t} \cos i \lambda \alpha(x+b) .
$$

Avremo

$$
\frac{d \varphi}{d x}=-i \lambda \alpha A e^{\lambda i} \sin i \lambda \mu(x+b) ; \frac{d^{2} \varphi}{d x^{2}}=\lambda \cdot \alpha^{2} \varphi ; \frac{d \varphi}{d y}=0 ; \frac{d^{2} \varphi}{d y^{2}}=0 .
$$

Ja condizione $\frac{d \varphi}{d x}=0$, per $x=-b$, ¿̀ soddisfatta; $\grave{e}$ inoltre $\Delta_{2 \varphi}=\lambda^{2} \alpha^{2} \varphi$. Dalla [11] avremo quindi

$$
a=\frac{1}{\sqrt{g h}}
$$

Per la parte meno profonda, posto

$$
\varphi=A^{\prime} e^{\lambda t} \cos i \lambda a^{\prime}\left(x-b^{\prime}\right),
$$




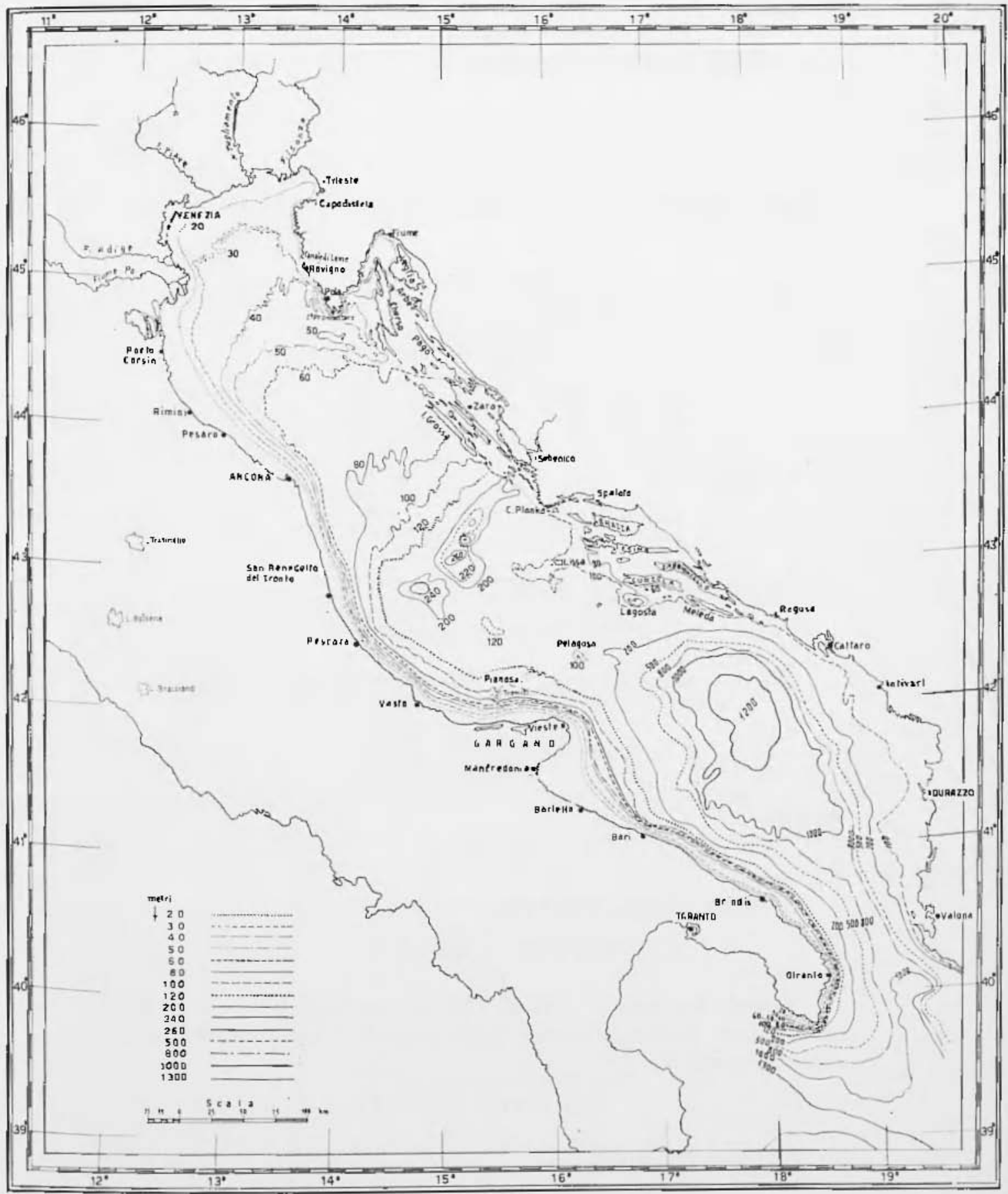

Fig. 8 - L'Adriatico, con alenne linee batimetrichc.

Fig. 8 - The Adriatic Sea, with some bathymetric lines. 
soddisfatte le condizione wi limiti, si ottiene parimenti

$$
a^{\prime}=\frac{1}{1 g h^{\prime}}
$$

Ta $\varphi$, come funzione continua assume uguali valori da una parte e dall'altra di $x=0$. Ne segue

$$
A \cos i \lambda a b=A \cos i \lambda \alpha^{\prime} b^{\prime}
$$

Attraverso $x=0$, anche $h \frac{d r}{d x}$ dev'essere continua:

$$
\left(h \frac{d \varphi}{d x}\right)_{x=0}=\left(h, \frac{d \varphi}{d x}\right)_{x=0}
$$

Se segue

$$
h \alpha A \sin i \lambda a b=-h^{\prime} \alpha^{\prime} A^{\prime} \sin i \lambda \alpha^{\prime} b^{\prime}
$$

Dividendo membro a menbro le [14], [15], si ottiene

$$
\text { ahtang } i \lambda a b=-a^{\prime} h^{\prime} \operatorname{tang} i \lambda c^{\prime} b^{\prime} \text {. }
$$

Pertanto, per $h^{\prime}<h$, ì $\alpha^{\prime} \neq \alpha$; e poiché, per le [12], [13],

$$
\frac{a^{\prime} h^{\prime}}{a h}=\sqrt{\frac{h^{\prime}}{h}},
$$

ne segue

$$
a^{\prime} h^{\prime}<\alpha h .
$$

In ralore assoluto, è pertanto

$$
\mid \text { tang } i f a b|<| \text { tang } i A a b^{\prime} b^{\prime} \mid \text {. }
$$

Fssenda $\lambda$ immagiuario (pug. 170 ), gli argomenti $i \lambda \alpha b$ e $i \lambda \alpha^{\prime} b^{\prime}$ sono reali; quindi, poiché al crescere delle tangenti, i coseni decrescono, in valore assoluto is

$$
|\cos i \lambda a b|>\left|\cos i a^{\prime} b^{\prime}\right|
$$

Ne segue, per la [14],

$$
A^{\prime}>A
$$


Cioè, lampiezza dell'onda libera risulta maggiore nella parte del bacino di minore profonditi.

Lungo il canale prima considerkto, esaminiamo ora la propagazione di un'onda forzata. Come ill precelenza, si abbia per $x<0$ una profondità $h$ e per $x>0$ una profondità $h^{\prime}$, sicché per $x=0$ la profonditì passi bruscamente da $h$ a $h^{\prime}$ (fig. 10). Per approssimarci al problema

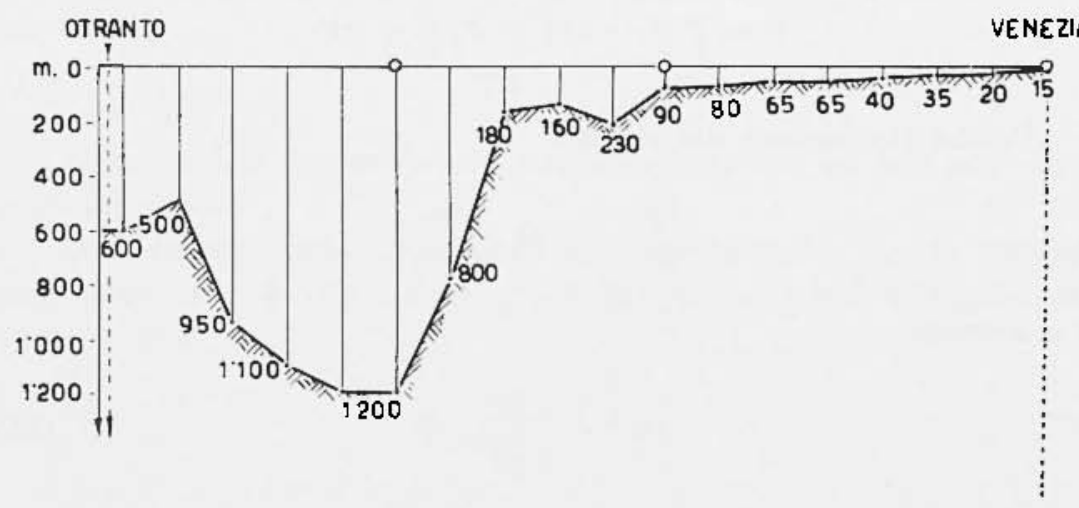

Fig. 9 - Andamento della profonditì dell'A striatico, lungo la linea di valle. ${ }^{35}$ )

Fig. 9 - Trend of elepth of the Adriatic along the elepth line. ( ${ }^{35}$ )
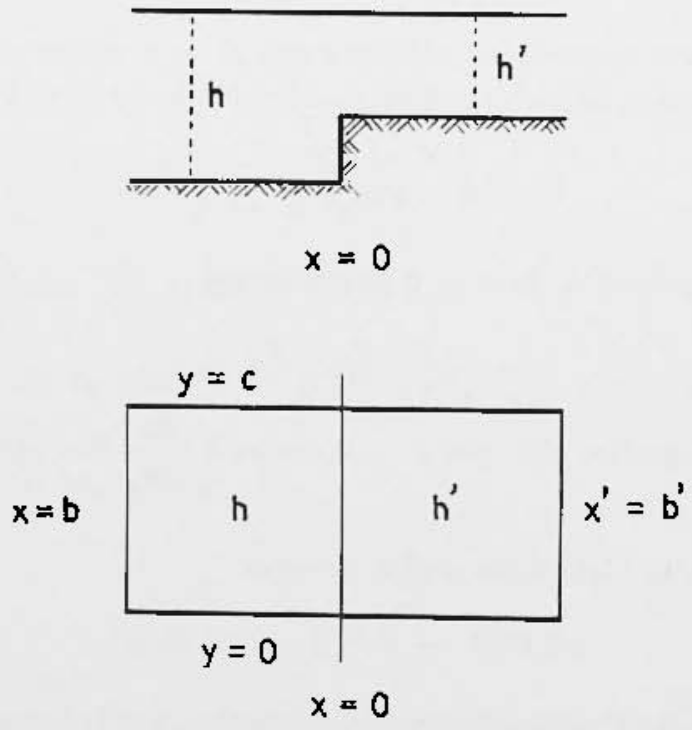

Fig. 10 - Sezione e proiezione di un canale con fondo a gratino.

Fig. 10 - Scetion and projection of a canal with a step-shaped buttom. 
delle maree, tali profondità vanno ritenute infinitamentc piccole, nei confronti della lungliezza d'ond

Consideriamo nel tratto $x<0$ un'onda propagantesi nel senso delle $x$ positive. Incidendo contro la soglia $x=0$, quest'onda subirà una riflessione parziale. Sel primo tratto, il movimento dipenderà quindi da una funzione

$$
p=F^{t}(t-a x)+F_{1}(t+a x)
$$

Poiché l'equazione del moto ì

$$
\frac{d-\varphi}{d t^{2}}=y^{2} \frac{d-m}{d x^{2}}
$$

in $\varphi$ avremo

$$
a=\frac{1}{g h} .
$$

Nel tratto oltre la soglia $x=0$, si propagherà l'onda residua

$$
\varphi=F_{2}\left(l-a^{t} x\right),
$$

eon

$$
a^{\prime}=\frac{1}{\sqrt{g h^{\prime}}} .
$$

Per la continuith, in $x=0$ dovrà essere

$$
F^{\prime}+F_{1}=F_{2}
$$

Si prova inoltre che, per $x=0$ anche $h \frac{d \varphi}{d x}$ dev'essere continua.

Per cui, d'ambo i lati della soglia avremo

$$
\alpha h\left[F^{\prime}(t)-F_{1}^{\prime}(t)\right]=\alpha^{\prime} h^{\prime} F_{2}^{\prime}(t),
$$

dove l'apice sulle $F$ indica derivazione rispetto ad $x$. Integrando si ottiene

$$
a h^{\prime}\left(\bar{F}-F_{1}\right)=\alpha^{\prime} h^{\prime} F_{2}^{\prime}
$$


Questa relazione, combinata con la [18], consente di determinare $F_{1}$ e $F_{2}$, una volta assegnato $F$. D'altronde, dalle [16], [17] si trae

$$
\frac{a^{\prime} h^{\prime}}{a h}=\sqrt{\frac{h^{\prime}}{h}} .
$$

Pertanto, per $h^{\prime}<h$ dalla [19] consegne $F-F_{1}<F_{2}$; e quindi, per la [18]

$$
F_{2}>F
$$

Il movimento assume perciò maggiore ampiezza nel lato meno profondo del canale.

Consideriamo infine, propagantesi Iungo lo stesso canale, un'onda progressiva. Per la [11], l'equazione di propagazione dell'onda puó essere scritta

$$
g h \frac{d^{2} q p}{d x^{2}}=\lambda^{2} \varphi
$$

dove, nel tratto più profondo (di profondità $h$ ),

$$
p=B e^{i \mu x+\lambda t}+B^{\prime} e-i \mu x+\lambda t
$$

mentre, oltre la soglia $x=0$, che immette nel secondo tratto, sul prolungamento del primo (nel nostro caso, la fossa Alriatica clie si prolunga nell'alto Adriatico)

$$
\varphi=B^{\prime \prime} e^{i \mu^{\prime} x+\lambda t}
$$

Tntanto, dalla [21] si ha

$$
\frac{d \varphi}{d x}=i \mu\left(B e^{i \mu x}-B^{\prime} e^{i \mu x}\right) e^{\lambda t} ; \frac{d^{2} \varphi}{d x^{2}}=-\mu^{2} \varphi
$$

Dalla $[20]$ si ha, allora

$$
-\frac{\mu^{2}}{\lambda^{2}}=\frac{1}{g h} .
$$

Dalla [22] risulta

$$
\frac{d \varphi}{d x}=i \mu^{\prime} B^{\prime \prime} e^{i t^{\prime} x+\lambda} ; \frac{d^{2} p}{d x^{2}}=-\mu^{\prime 2} B^{\prime \prime} e^{i \mu \mu^{\prime} x+\mu t}
$$


e quindi, per la [20],

$$
-\frac{\mu^{\prime 2}}{\lambda^{2}}=\frac{1}{g h^{\prime}}
$$

per cui

$$
\frac{\mu}{\mu^{\prime}}=\sqrt{\frac{h^{\prime}}{n_{n}}} .
$$

La prima condizione di continuità esige che le [21] e [22] si nguaglino per $x=0$; ne viene

$$
B+B^{\prime}=B^{\prime \prime} \text {. }
$$

La seconda condizione di continuità, in corrispondenza della soglia $(x=0)$, si traduce nell'uguaglianza

$$
h \frac{d \phi}{d x}=h^{\prime} \frac{d p}{d x}
$$

quindi, dalle [23], [24] risultat

$$
\frac{\mu}{\mu^{\prime}} h\left(B-B^{\prime}\right)=h^{\prime} B^{\prime \prime}
$$

da cui, per le $[25]$ e [26],

$$
2 B=B^{\prime \prime}\left(1+\sqrt{\frac{h^{\prime}}{h}}\right)
$$

e infine

$$
B^{\prime \prime}=\frac{2 B}{1+\sqrt{\frac{h^{\prime}}{h}}} .
$$

Quindi, per $h^{\prime}<h$ è $B^{\prime \prime}>B$.

Pertanto, l'onda propagantesi nel tratto poco profondo, ha un'ampiezza maggiore dell'onda incidente nel tratto profondo.

Concluilento, un sistema di onde lunghe, comunque si propaghi verso l'alto Adriatico, si presenteri presso le coste venete con un'anpiezza maggiore di quella con cui ha percorso l'Ailriatico meridionale. 
6. - SULL'EFTETTO DI RIsONANZA IN UN'" ACQUA ALTA $n$.

Consirleriamo ora un'altra causa di alterazione dinamica del livello del mare, legata al transito di una variazione di pressione atmosferica.

I giapponesi H. Yamada, J. Okabe e M. Kumazawa hanno provato (32) che, quando un'intumescenza liquida, conseguente ad una perturbazione atmosferiea di intensita invariabile - che avanza, con velocità costante, alla superficie dell'acqua -, monta lungo un letto inclinato avvicinandosi alla riva, quanto più piccola d̀ la pendonza del fondo tanto maggiore $\dot{e}$ il livello doll'onda: la crescita improvvisa si manifesta quando la variazione della pressione transita sopra i] punto di risonanza, definito rispetto alla profonditì dell'ncqua.

Se $P-P(X)$, dove $X=x-V t$, esprime l'intensità della perturbazione della pressione atmosferica agente sulla superficie dell'acqua, su cui si propaga con velocita $V$ (x definendo, con $y=0$, la media posizione della stuperficie liquida), si lia

$$
h(x)=h_{o}\left[1-\lambda \operatorname{tangh} \cdots \frac{x}{L_{2}}\right],
$$

dove $h_{0}$ esprime la profondità nel punto $x=0$, $\lambda$ ì una costante, $T_{\text {? }}$ la lunghezza della zona in declivio. Si prova che la risonanza delle onde destate dal passaggio della perturbazione, inizia esattamente alla profondità $h_{0}$, per la quale risulta

$$
\sqrt{g h_{o}}=V
$$

$A 1$ crescere dell'estensione $2 L_{1}$ della perturbazione $\left(-L_{1}<X<L_{1}\right.$ ), resce evidentemente la zona interessata dalla mareggiata; e quanto più $L_{2}$ atimenta, tanto più dolce si fa la pendenza del fondo, fra duo profondità fisse $h_{1}$ e $h_{2}$ ( $h_{1}$, al largo; $h_{2}$ presso la costa).

La superficie $e$ il fondo dell'acqua sono rappresentate rispettivamente da

$$
y=\eta(x, t), \quad \text { e } \quad y--h(x)
$$

Se $u$ ̀̀ la componente orizzontale della velocita del liquido, sono da soddisfare le equazioni differenziali $\left({ }^{33}\right)$ 


$$
\begin{gathered}
\frac{\partial u}{\partial t}+u \frac{\partial u}{\partial x}=-g \frac{\partial}{\partial x}(\eta+P), \\
\frac{\partial \eta}{\partial t}=-\frac{\partial}{\partial x}\{u(h+\eta)\} .
\end{gathered}
$$

Fissato il campo di variazione di $P$

$P=-P_{o}\left\{1+\cos \left(\pi \frac{x-V t}{L_{1}}\right)\right\} \quad$ per $\frac{x-V t}{L_{1}} \leqslant 1$, (diversamente $P=0)$ gli AA. compiono una serie di calcoli, con i seguenti dati di partenza:

$$
P_{\mathrm{o}}=0,10 \mathrm{~m}, L_{1}=10 \mathrm{~km}, V=15,3362 \mathrm{~m} / \mathrm{sec} ;
$$

$$
h_{o}=24 \mathrm{~m}, h_{1}=h_{\circ}(1+\lambda)=40 \mathrm{~m}, h_{2}=h_{o}(1-\lambda)=8 \mathrm{~m} \text {, }
$$

essendo $\lambda=\frac{2}{3}$. Fatto $g=9,80 \mathrm{~m} / \mathrm{sec}^{2}$, si ha

$$
\frac{V^{2}}{g h_{o}}=1,00, \frac{V^{2}}{g h_{1}}=0,60, \frac{V^{2}}{g h_{2}}=3,00 .
$$

I risultati sono riassunti in due tabelle e in due figure. $A$ noi interessa qui riportare la figura che dà il variare di $\eta$ - cioci dell'elevazione della superficie — in $L_{1}$, al variare di $h$ (fig. 11). Si veda il notevole efletto sull'ampiezza del moto ondoso, quando $m$

$$
m=V /\left(g h_{1}\right)^{1 / 2}=\left(h_{o} / h_{1}\right)^{1 / 2}
$$

tende a 1 , sia per valori decrescenti che per valori crescenti: l'acqua alta clie accompagna la bassa pressione, cresce al tendere di $m$ a 1 e, transitata su $m=1$, costituisce l'onda libera dietro la bassa pressione; la sua tendenza è di gonfiarsi a mano a mano che ascende il fondo inclinato ed essa diverrà via via più ampia, quanto più ampia ê l'onda primitiva. L'effetto di risonanza diverrà più pronunciato, quando le condizioni prossime allo stato di massima amplificazione, si estendono al massimo prima e dopo il punto stesso di risonanza; e quindi, allorché il trasferimento dallo stato suberitico a quello supercritico è raggiunto sopra un fondo esteso il più doloemente inclinato. Nella fig. 12 sono riportati i risultati dei calcoli - eseguiti con un calcolatore elettronico dell'Università di Kyoto - condotti con i dati di partenza inclusi in [27]. I valori di $P, u$ e $\eta$ al tempo $t=0$ sono ricostruiti rispettivamente in 
(A), (B) e (C) sul punto-tempo zero (S. 0). Te linee intere in (B) e (C) mostrano $u$ (velocitì della corrente) e $\eta$ (elevazione della superficie) in corrispondenza di punti-tempo appropriati. Ne risulta che il massimo della mareggiata o la massima velocita di corrente si riscontrano quando la pertubazione si approssima all'estremo superiore del fondo inelinato. I'efietto ottenuto, come risultante dell'azione del fondo inclinato e della profondità di risonanza, va in realtà considerato come singolo, l'inclinazione e la profondità di risonanza essendo concettualmente inseparabili.

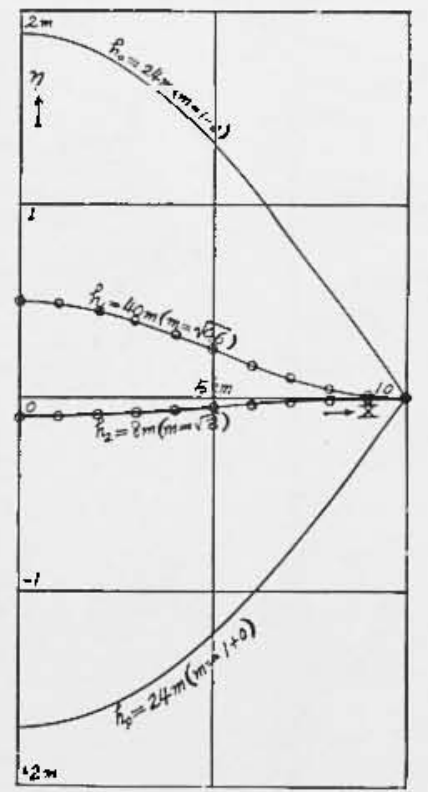

Fig. 11 - Distribuzione degli innalzamenti di livello nol easo contemplato in [27] - Seconto Yamnda cd al.

Fig. 11 - Distribution of level raisings in the case considered nnder [27] - After Yamada and others.

Va comunque sottolineato il fatto che tanto maggiormente eresee lampiezza dell'clecazione del livello dell'acqua quanto piu l'inclinazione del fondo si fa graduale. Pertanto, il fenomeno di risonanza non è un evento, che si verifica in un punto singolo, isolato; esso va invece inteso come un efỉetto d'insieme sopra un più o meno largo dominio. Gli $\mathrm{A} A$. eitati, nella parte II ( $\left.{ }^{34}\right)$ del loro lavoro, provano che l'efretto della viscosità è traseurabile ai fini dei fenomeni ai risonanza; inoltre, l'acqua 


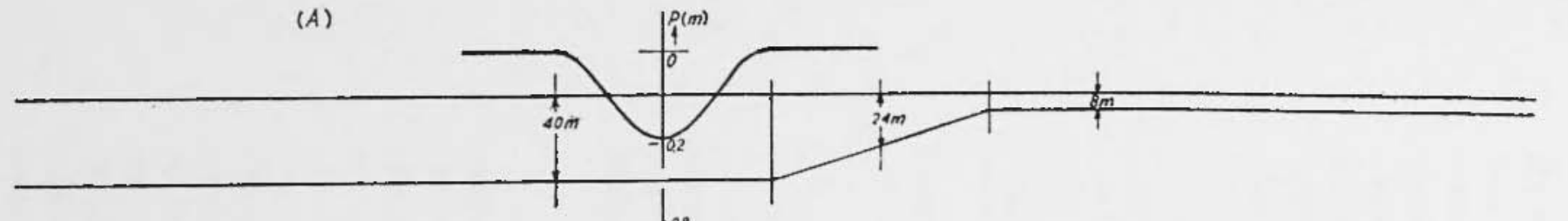

(B)

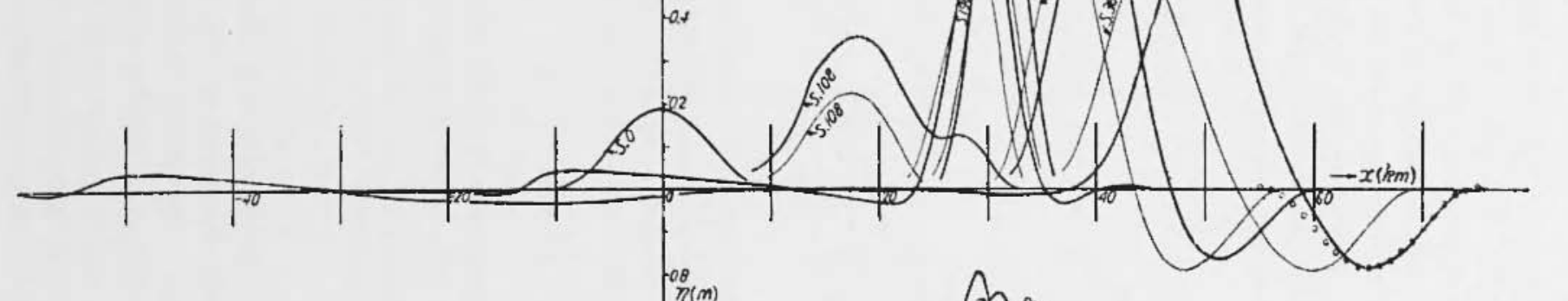

(C)

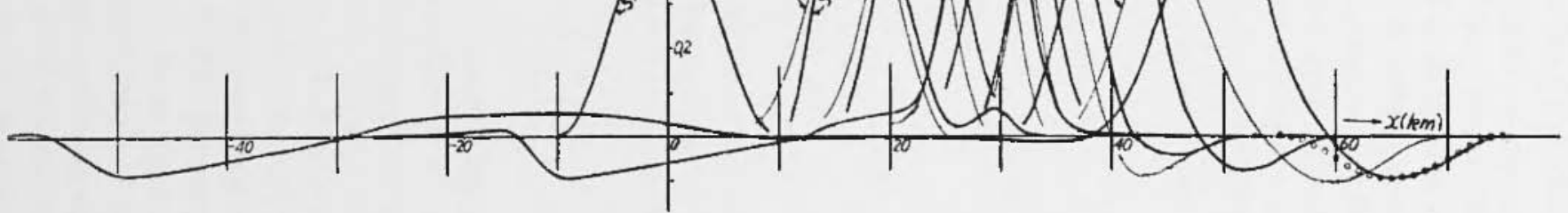

Fig. 12 - Fondo inclinato ed acqua alta per risonanza: linea grossa per caso non lineare, linea sottile per approssimazioni lineari (v. testo) - Secondo Yamada od al.

Fig. 12 - Sloping bottom and high water due to resonance: the thick lines representes a non linear case, the thin lines a linear approximated case (see text) - After Tamalla and others. 
alta provocata da una depressione di dimensioni nettamente maggiori nei confronti della scala lineare del fondo inclinato, non determina modificazioni sensibili (v. p. es. fig. 5). Infine, dimostrano che gli effetti dei fattori meteorologici possono essere espressi unicumente in termini di un gradiente di pressione atmosferica. Così, linfluenza del vento [valutata, p. es., per quanto conecrne l'Adriatico, da F. Stravisi (25)] può pure essere inclusa nello stesso schema di calcolo. In altre parole, lo stesso tipo di soluzione approssimata i valiulo per una pertubazione meteorologica, presi in senso globale.

Vedremo che l'efleteto considerato interessa molto da vicino il golfo di Venezis.

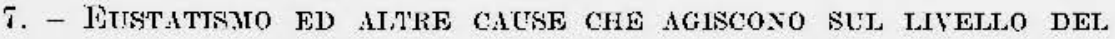 MARE.}

Dopo i richiłmi teorici, di cui ai numeri precedenti, torniamo alla situazione fra terra e mare nella zona di Veneziat. Tale situazione dipende dai movimenti delluma o dell'altro, oppure - come generalmente avviene - di entrambi. Siché il graluale aumento o diminuzione del dislivello fra caposaldi a terra e livello medio del mare $(l m m)$ è il risultato dei movimenti relativi dell'acquin e del suolo.

Attumente, il $l m m$ i in generale ammento sul nostro pimetr. Isa causa d̀ attribuita al progressivo necrescimento della temperatura iell'aria nell'ultimo secolo (almeno fino al 1940) e al conseguente aumentato scioglimento dei ghincei. Questo accrescinsento intrinseco, indicato anche con il termine " eustatismo ", è stato valutato sulla merlia li 1,1-1,2 mm/anno per l'ultimo trentennio.

Tali valutazioni sono estremamente delicate. Esse si valgono prevalentemente dei dati mareografici: le postazioni mareografiche risentono nafuralmente di pertubazioni locali, clıe possono mascherare come in efletti mascherno - l'andamento del fenomeno, nei suoi aspetti montiali.

Di qui inevitabili incertezze, non sceve da autentiche contraddizioni. (*)

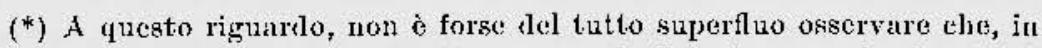
aleune grancli città costiere, sede di mareofrafi, il carico complessivo degli exlifiei si è notevolmente aceentuato negri ultimi decenni, iu serguito a massieci pesanti insediamenti industriali. Il conseguente avvallamento del terreno, quantu entra nel livello segnato dai mareografi? 
$\Delta$ questo riguardo, ritengo utile richiamare l'attenzione sui risultati di uno studio condotto da William I. Donn e J)avid M. Shaw, del Lamont Geological Observatory $\left({ }^{36}\right)$, in eui il livello marino nell'ultimo sccolo è correlato all'andamento della temperatura dell'aria. Sulla

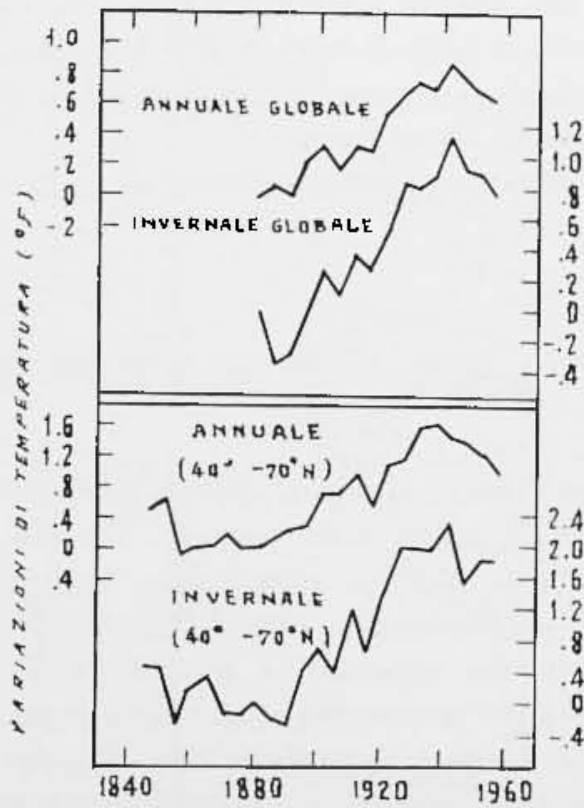

Fig. 13 - Andamento delle medie temperature mondiali globali (sopra, secondlo Mitchell) e per le latitudini da $40^{\circ}$ a $70^{\circ} \mathrm{N}$ (sotto, sceondo Donn e Shaw), prima e dopo il 1940.

Fig. 13 - Trend of overall average world temperature (top, after Mitehell), and for lititudes from $40^{\circ}$ to $70^{\circ} \mathrm{N}$ (bottum, after Donn and Shaw), before and after 1940 .

base di studi condotti da Willett $\left({ }^{37}\right)$ e proseguiti da Mitchell $\left({ }^{38}\right)$, un riscalilamento annuo globale si verificò clal 1880 al 1940, per l'ammontare di $0,8^{\circ} \mathbf{F}\left(0,4^{\circ} \mathrm{C}\right)$; nello stesso periodo, l'innalzamento termico globale invernale fu di $1,2 \circ \mathbf{F}(0,6 \circ \mathrm{C})-$ fig. 13 , parte superiore - La parte inferiore della fig. 13 mostra un innalzamento termico alle latitudini da $40^{\circ} \mathrm{N}$ a $70 \circ \mathrm{N}$ di maggiore ampiezza: un risealdamento annuale di $1,6^{\circ} \mathrm{F}\left(0,9^{\circ} \mathrm{C}\right)$ e un'elevazione termica invernale di $2,4^{\circ} \mathrm{F}\left(1,3^{\circ} \mathrm{C}\right)$.

Dall'inizio del 1940, il fatto nuovo: un'inversione dell'andamento termico. Il moderato raftreddamento che ne è scguito, ha compensato 
circa il $30 \%$ dell'aumento di temperatura, verificatosi fra il 1880 e il 1940 .

Un effetto simile si è verificato in un altro parmetro climatico: la pioggia. E stata constatata una rliminuzione delle precipitazioni nei primi 40 anni di questo secolo, seguita da un aumento alla pioviosita dopo il 19.10. Un riflesso del predetto andamento termico si è avuto pure sul livello dei mari. Donn e Shaw hanno elaborato le osservazioni mareografiche di nove stazioni delle coste atlantiche degli Stati Uniti, suclivirlendole in due serie: nna dall'inizio delle osservazioni al 1939 e l'altra dal 1940 al 1960; e calcolando con il metorlo rlei minimi quarlrati, l'andamento rettilineo di ogni serie, per ogni stazione (fig. 14). Tutte le stazioni, eccetto Portland, mostrano una tendenza all'innalzamento fino al 1940 circa.

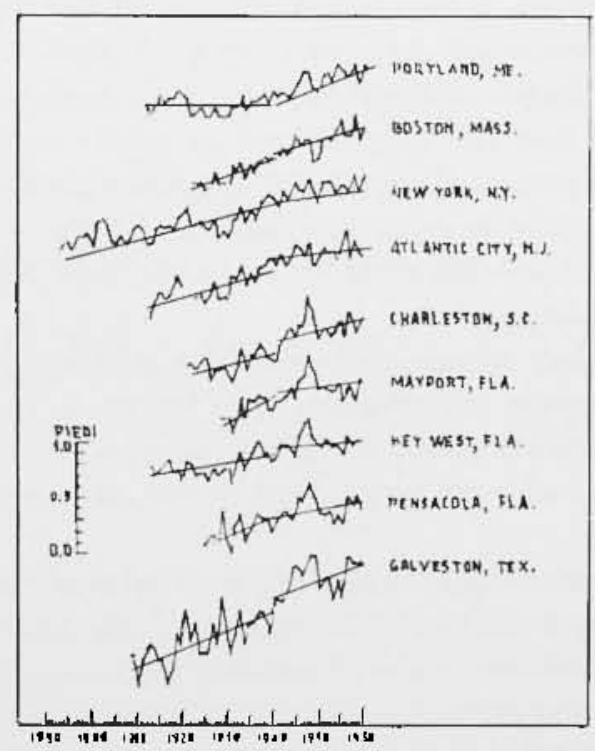

Fig. 14 - Andamento del livello marino per aleune località allantiche americane prima e dopo il 1940 (secondo Donn e Staw).

Fig. 14 - Trend of sea level for soute American places on the Atlantic seabord before and after 1940 (after Donn and Shaw).

Dal 1940, tutte le stazioni, ad eccezione di Portland, Charleston e Galveston, accennano al una decisa diminuzione nell'entità dell'innalzamento del livello del mare, benché l'ascesa continui in tutte le nove 
stazioni. Portland è chiaramente anomala, Clarleston lamenta incompletezza di dati prima alel 1942 , mentre in altre stazioni agiscono fattori locali, natumli e occasionalmente artificiali. Prescimlendo da Portiand e Charleston, la metia ascesa del livello del mare presso le altre setite stazioni, prima del 1940, fu di $0,7 \mathrm{ft}(21 \mathrm{~cm})$ per cinquantennio; da. 1940 al 1960 l'aumento del livello marino $\mathrm{fu}$ in media di $0,4 \mathrm{ft}(12 \mathrm{~cm})$ per cinquantenuio, il che rappresenta un $40 \%$ di diminuzione nella temlenza all'innalzamento. T'espansione termica degli strati superficiali dell'Ocenuo (per mo spessore di $400 \mathrm{~m}$ circa), può tar ragione tli circa un centimetro soltanto dei cambiamenti osservati. L'aumento nel livello viene attribuito quasi totalmente allo scioglimento dei ghiacei nelle alte latitudini, per cui la diminuzione della temperntura a partire tal 1910 ha avuto come conseguenza una diminuzione nello seioglimento dei ghiacei, il cle sembra costitnire nua convincente spiegazione della diminuita entitì nella crescita del livello del mare.

Come suggeriscono gli $\Lambda \Lambda$. citati, va però sottolineato il fatto che i valori della temlenza all'innalzamento qui riportati non sono tipici del livello marino mondiale e quindi non possono essere totalmente atribuiti allo scioglimento dei ghiacci. Infatti, l'elaborazione dei dati mareografici, ottenuti lungo le coste statunitensi del Pacifico mostrano un innalzamento del livello del mare che è circa la meta di quello osservato luugo le coste atlantiche.

Necessita quindi grande cantela nel far previsioni sull'antamento dell'eustatismo, speeie con riferimento al fenomeno in atto dell'inversione nella temperatura media del globo, di eui d'altronde non si trova traccia nelle relazioni e nei lavori degli esperti, impegnati nel problema di Venezia.

Ha nou si fermano qui le cause cle possono contribuire a variazioni del livello marino. Intanta è stato osservato che il livello del mare in ogni emisfero è alto thurante il rispettivo autunno e basso dlurante la primavera $\left({ }^{(9,40}\right)$; una prima conchsione, attribuisce la principale teviazione dal livello medio alle variazioni di densità nelloceano (v. 9.3). Anche la pressione atmosferica la un sensibile efletto, maggiore nelle alte latitudini nei confronti delle basse. Così, eome per l'oceano Pacifico anche per il Ford Atlantico (fig. 15), l'efietto sulle variazioni di livello, della temperatura dell'acqua e della salinità è molto meno importante cli quello della pressione atmosferiea ${ }^{\left({ }^{41}\right)}$. Ben diversamente si presentano i fenomeni alle basse latitudini. Uno studio condotto sulle variazioui tlel livello marino alle Bermude, ha mostrato $\left({ }^{41}\right)$ che l'onda di temperatura massima nelloceano, progredisce verso il basso di 


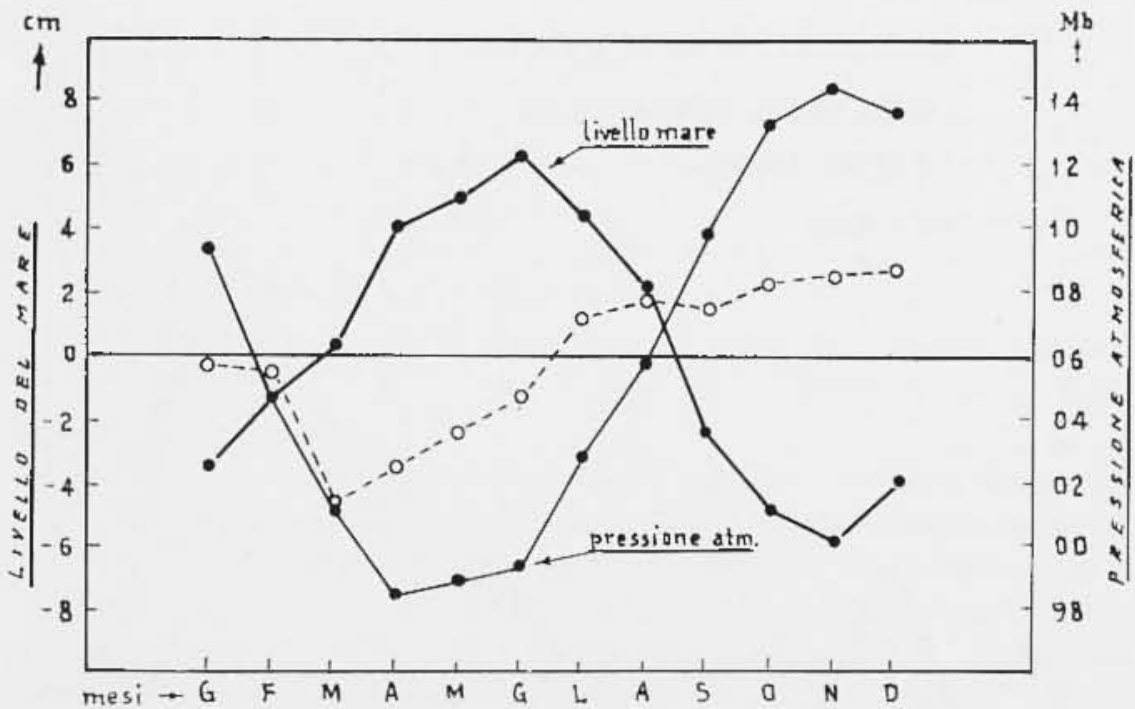

Fig, 15 - Jivello marino medio associato a variazioni della pressione atmosferica, (linea trattegrgiata, livello resiluo) a Grindavik, sulle coste meridionali dell'Islanda (secontlo Slatw e Donn).

Fig. 15 - Mean sea level referred to atmospheric pressure variations (dashed line, resilual level) at Grindavik on the South coast of Iceland (after Shaw and Donu).

circa $40 \mathrm{~m}$ per mese e porta la sua più profonda penetrazione a circa $200 \mathrm{~m}$ fra Gennaio e Febbraio. Durante i mesi più caldi, un termoclino superiore, mediumente a ea $80 \mathrm{~m}$ di profonditì, lentamente si appiattisce con la propagazione verso il basso dell'onda ternica. Un particolareggiato lavoro di calcolo, condotto sui dati di temperatura, salinità, densità e anomalia dinamica (intesa come la correzione in centinctri per ia conversione di profondità misurate in profondità vere), hanno provato che variazioni isostatiche fondamentali si manifestano solo nei $2.000 \mathrm{~m}$ superiori.

Un'ulteriore, complessa indagine ${ }^{41}$ ), condotta sulle osservazioni mareografiche ottenute a Bermula dal Giugno 1954 al Maggio 1962, hanno portato ai risultati sintetizzati nella fig. 16, da dove risulta che, per la zona delie Bermude, l'efletto predominante ì il cosi detto "steric 


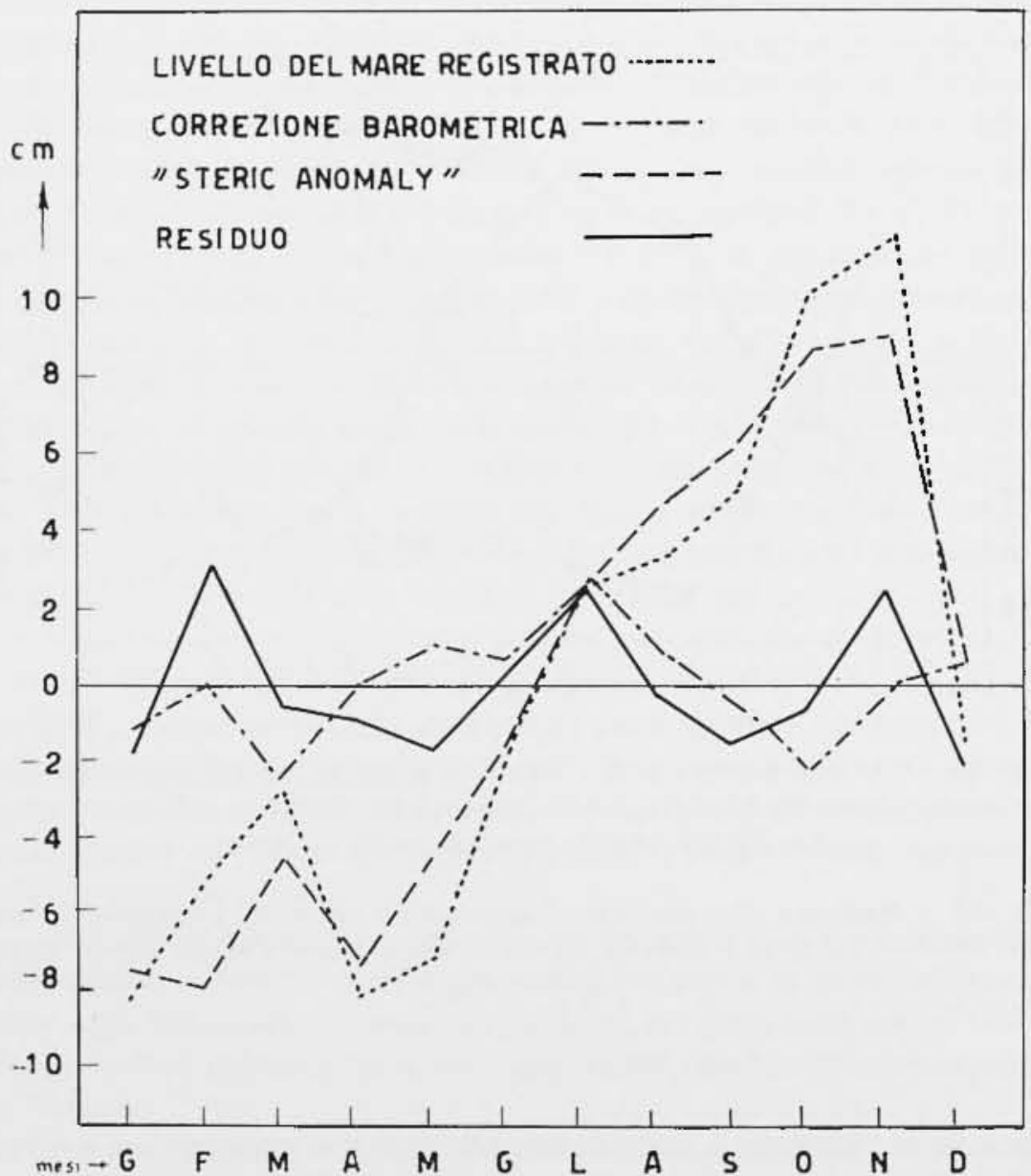

Fig. 16 - Medie mensili livello marino per il periodo 1958-1962 presso bermuda - sceondo Shaw e Domin. (Lit "steric anomaly" esprime la deviazione dal livello medio annuale, dovuta a variazioni nel volume specifico dell'aequa).

Fig. 16 - Acan monthly sea level for the 1958-1962 period near the Bermula lslands - after Shaw and Donn. (The "sterie anomaly" expresses the deviation from the meau innual sea level due to variations of the specifie water volume). 
effect ", cioc il cambiamento ali livello causato dalle deviazioni dalle profonditn di pressione normale, provocate dalle espansioni di volume.

Pertanto, i risultati delle ricerche sul livello del mare prosso le Bermude e presso l'Islanda, confermano precedenti conclusioni, tratte da studi concernenti l'Oceano Pacifico: le variazioni di livello marino sono cioc dovute, per una parte preponderante, nelle basse latitudini da "steric effocts" (legnti ut momalie dinamiche associate all'onda termica) e, nelle latitudini elcvate, dugli effetti burometrici. Queste conclusioni sono tratte sulla base del contrasto fra il regime termico zonale anmuale negli oceani e le annuali variazioni di pressione nella atmosfera.

Nia non è tutto. Altre complieazioni nascono dal fatto che anche gli Occani sono animati da oscillazioni libere. I'Oceano Atlantico settentrionale, p. es., presenta sensibili oscillazioni libere, delle quali recentemente furono studiate le caratteristiche dei tre primi modi gravitazionali, aventi periodi ali 21 , It e 11 ore rispettivamente, e stutture di uno, due e tre sistemi nnfidromici ( $\left.{ }^{25}\right)$.

E vero che i fenomeni accennati, in una stima pluriennale, vengono attenuati nei loro effetti. Permangono però dei residui, non facilmente valutabili. Inoltre, il fatto che essi siano stati rilevati per gli Oceani, non esclucle possano intercssarc, in misura più o meno accentuata, anche i mari interni, specie se - come il Hediterranco (*) - presentano caratteristiche di tipo oceanico.

Si deve pertanto concludere che l'esatta valutazione, per sclezione, delle variazioni di livello marino sono tutt'altro che agevoli. Nel momento attuale, poi, ogni previsione viene resa più alentorin dall'inversione della temperatura del globo, a cui si è fatto cenno sopra.

Ritorniamo all'Adriatico.

Sulla base dei dati riportati da MIosetti ( ${ }^{(4)}$ ) (Tab. I, p. 246), ho voluto determinure l'andamento del $l m m$, durante gli ultimi decenni, per porti della sponda dalmata e istriana. Le figg. 17, 18 riportano, in grafico, i valori del $l m m$ per Ragusa, Spalato e Buccari sulla sponda dalmata, e di Pola e Rovigno per la sponda istriana. I tratti di retta segnati punto e linea - sono stati calcolati con il metodo dei minimi

(*) S. Polli ( $\left.{ }^{13}\right)$, per es., valuta per il livello molio del Stediterraneo, oscillazioni annuali simultute, con un massimo in Noveubre e un minimo in Gennaio. J'ampiezza è ili circa $11 \mathrm{~cm}$. 


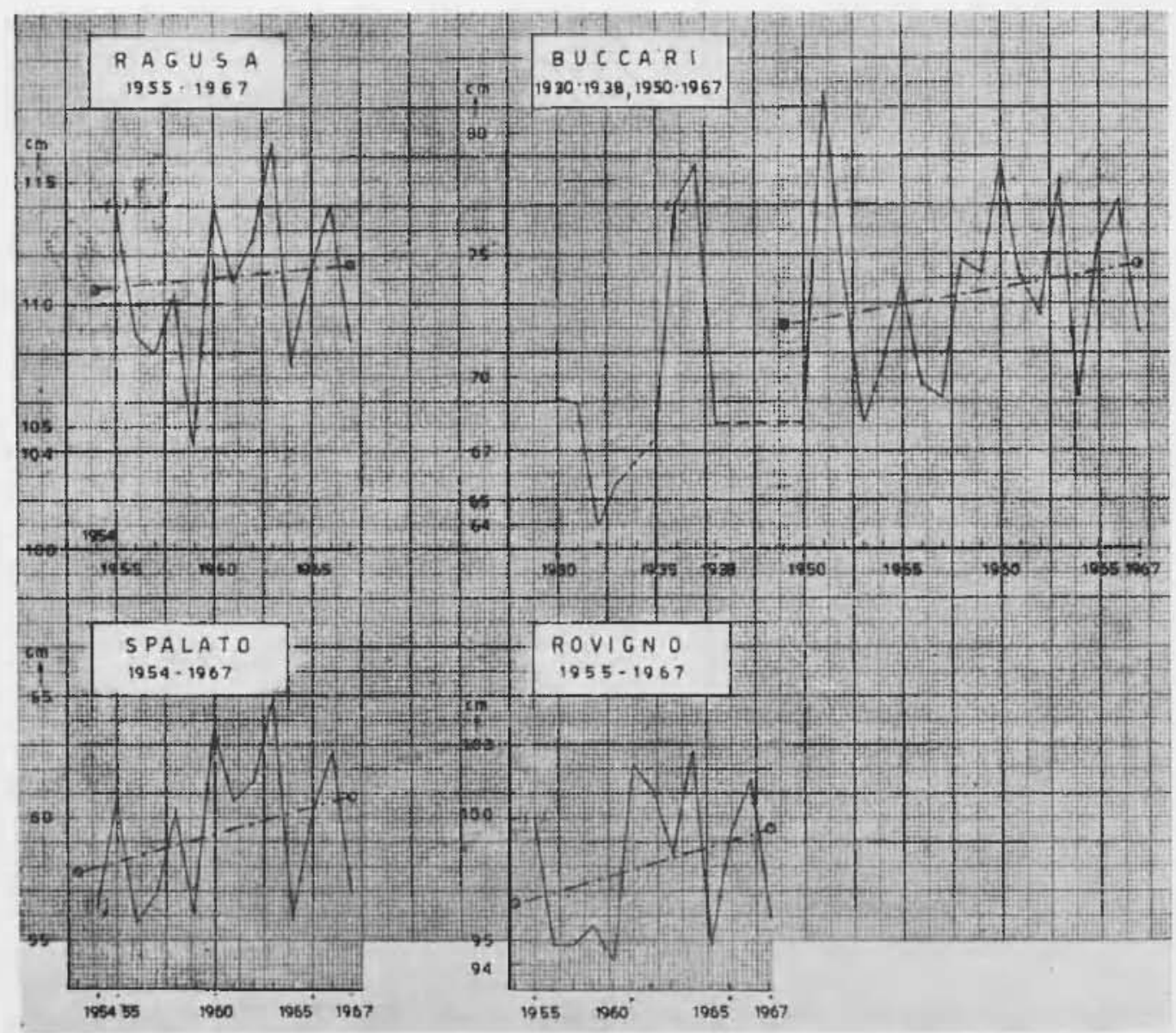

Fig. 17 - Livello medio marino a Ragusa, Spalato, Buceari e Rovigno, per brevi periodi di registrazione, mediato (junto e linea) con il metodo dei ninimi quadrati.

Fig. 17 - Mean sea levels of Ragusa, Spalato, Buceari and Rovigno, for short recording periods, averaged (dots and dashes) after the method of least-squares. 
quadrati. Va rilevato che $\mathrm{i}$ dati a disposizione erano troppo pochi, e con searti troppo accentuati, per poter consentire approssimazioni spinte: i risnltati, quindi, vanno ritenuti puramente indicativi.

Per Ragusa, in 13 anni, si trova un aumento medio annuo tel $l m m$ di mm 0,7t. Per Spalato, nel periodo di 14 anni esaminati, l'au-

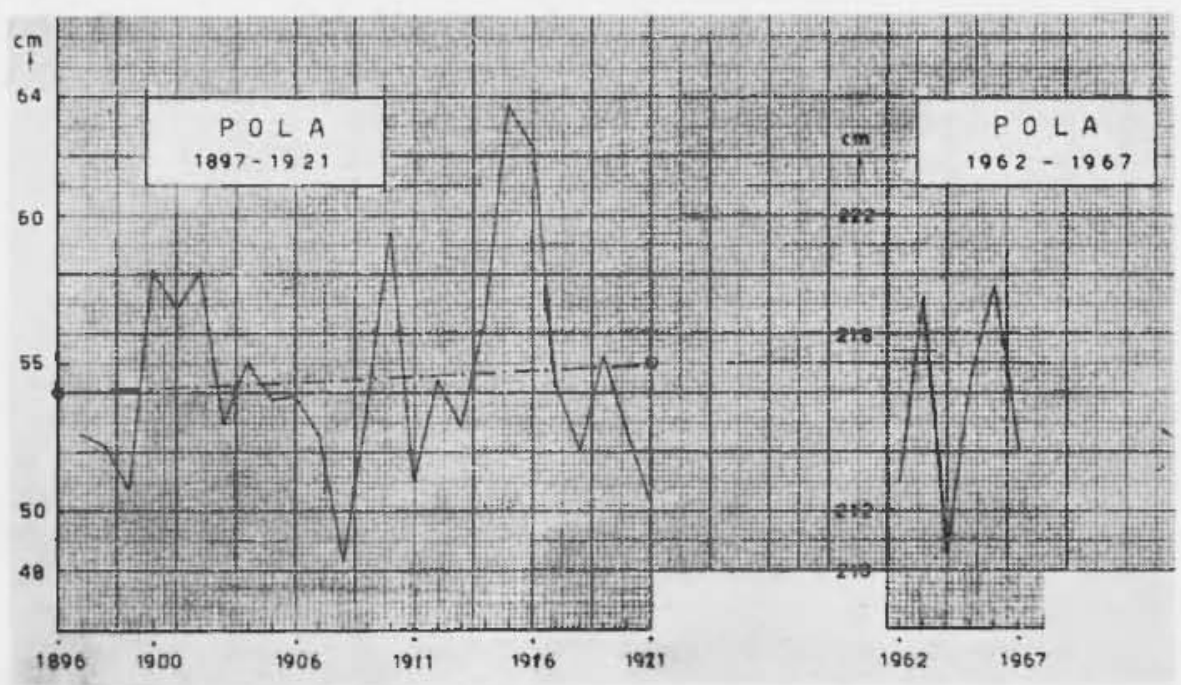

Fig. 18 - Anclamento del l.m.m. a Pola: tratti 1897-1921, 1962-1967. L'intervallo $189 \bar{t}-1921$ è stato mediato con il metodo slei minimi quadrati.

Fig. 18 - Devclopment of mean sea level at Pola for the intervals 1897 to 1921 and 1962-1967. The first interval has been averarged after the method of least-squares.

mento melio fu di mm 2,2/anno (Si noti la spiceata analogia dell'andamento del $l m m$ in Ragusa e a Spalato dal 1958 al 1967). Per Buccari (nel golfo del Quarnaro), l'aumento dal 1950 al 1967, fu di mm 1,35/anno. Per Rovigno d'Istria, dal 1955 al $196 \mathrm{~T}$, si ebbero mm 2,3 di aumento annuo. A Pola si ebbe il più lungo tratto di osservazioni mareografiche, dal 1897 al 1921 (riprese poi nel 1962) (*). Fino al 1921, l'aumento

(*) A proposito dello stazioni mareografiehe dalmate e istriane, ho notato con stupore, ehe in aleuni lavori ili esperti hamo periuto il loro millenario nome italiano, per assumerne uno sjavo: Dubrovuik in luogo di Ragusa (considerata, a ragione, la quinta Repubblica marinara italiana), 
medio osservato fu di soli $4 / 10$ di mm l'anno (*).

Come si vede, l'esito dei calcoli non si presta a conclusioni certe; senza dubbio, intervengono fenomeni locali, di effetto non facilmente apprezzabile. Mediamente, sembra di poter affermare che il livello medio, dal 1954 al 1967, abbia subito un incremento medio annuo maggiore, jassando da Ragusa a Rovigno. Quanto, su questi risultati, influisec l'aumento "ajpjarente" del livello medio, associato alle forti variazioni dinamiche del livello del mare, provocate dal transito di depressioni, specialmente sull'alto Adriatico?

\section{ABBAsSAMENTo DEL suolo, BRADisismo.}

8.1. - Riassunte alcune delle cause, responsabili dell'aumento del livello marino nel mondo, passiamo al fenomeno di abbassamento del

Split inveee di Spalato, Bakar per Buccari, Rijeea per Fiume, Pula per Pola, Rovinij in luogo di Rovigno, ... perfino Koper invece di Capodistria! (che, a tult'oggi, anche giuridicamente -- Memorandum di Jondra ì italiana).

Non so come si possa lavorare per la salvaguardia di Venezia e offendere questa città in ciò che ha di più vivo e greloso: la sua traslizinne millenaria, che ha fatto dell'altra sponda (dove fn preceluta da Roma) un tutt'uno con la Serenissima.

E non mi si faceia, per favore, la solita stolida aceusa di nazionalismo, cosi spesso a sproposito elargita in questi tempi di "scollamento" italico.

Qui è questione di un minimo di sensibilità e di giustizia storica. Sensibilità e giustizia che mossero perfino cittadini dell'impero Austro-ungarico, che conservarono a quelle localita il loro nome italiano, eome provano i riehiami biblingrafici $13,15,18$ e 19 . Ci volevano degli "italiani " per compiere tanto scempio. Evilentemente, per costoro il dramma rli Venezia è soltanto fonte ‘li lavoro. Non metto in dubbio che possano compiere egualuente opere egregie. A questo riguarko, però, Einstein ebbe ad affermare: "credo che l'amore sia masstro migliore di quanto lo sia il senso del dovere "; mentre, nella sua "Wcltgeschichte " Hegrel lasciò scritto: " mulla di grande al mondo si fa senza passione n. Entrambi preceduti dal grankle Jascal, per il quale "la conoscen a presuppone l'amore: si riusoiri a conoscere la veriti - a conoscerla realmente nel senso più profondo, con passione dell'assimilazione - nella misura che si aman.

Non amoro per Venezia è in costorn, ne tanto meno passione; ma neppure il resiluo tepore delle ceneri.

$\left(^{*}\right)$ S. Polli $\left(^{45}\right)$ ha rleterminato il valore della variazione secolare dei livelli marini, relativi ad 11 porti italiani. Il valore medio della variazione per i mari italiani corrisponsle ad un aumento del livello marino $1 \mathrm{li} 16 \mathrm{~cm} /$ secolo, contro gli $11 \mathrm{~cm} / \mathrm{secolo}$ di tntti i mari del unondo. 
stolo, al quale è intimamente legato l'apparente aumento del livello del mare a Venezia.

Un generale fenomeno di abbassamento, sembra investire l'arco alriatico, almeno dalle foci del Tagliamento a Ravenna; tale abbassamento è senz'altro efrettivo nella zona del Delta padano, dove ha ragginuto proporzioni eceezionali. Per quanto si riferisce al pelta, la principale (se non eschusiva) causa della flessione del terreno ì stata, a suo tempo, ben individuata: la disorlinata estrazione di acque metanifere. Di esse ho lungamente trattato in precedenti publslicazioni e qui non intendo ripetermi $\left({ }^{46,47}\right)$. Limitiamoci pertanto alla parte che piu ci interessa: quella comunque associata a Venezia.

Una comparazione attendibile di misure di livellazione eseguite in una determinata zona, richiederebbe linec di livellazione espressamente istituite, il più possibile in là nel tempo, e rimisurate ad intervalli regolari, con gli stessi metorli e con la stessa precisione. Tali condizioni non si sono di fatto verifiente per la zona di Venezia, prima del $1970{ }^{\left({ }^{4}\right)}$.

I'Tstituto Geografico Militare (I.G.M.) ha compinto alcune livellazioni di precisione durante gli ultimi 100 anni: una prima, iniziata negli ultimi flecenni del secolo seorso exl ultimata nel 1908, ma seconda nel 1925, una lines di alta precisione Genova-Venezia nel 1942, nna nuova rete altimetrica fondamentsle nel 1952 el una livellazione di alta precisione nel 1968. Fanno aggiunte le livellazioni eseguite da ditte private, per conto del Magistrato alle Acque nel 1961 e nel 1964.

Quindi, rilievi eterogenei (per precisione e per metodi), eseguiti in tempi alispauti, con un numero esiguo di capisali commi. In ogni modo, non spetta a me la critica dei risultati.

Mi limito a riportare il rafironto fra le varie livellazioni riferite al caposaldo di Punta della Dogana (S. Haria della Salute). Esso si riassume nei seguenti risultati: fra le livellazioni del 1942 e quelle del 1961, l'ab-

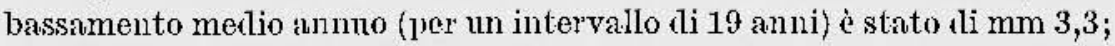
fra il 1961 e il 1968 (intervallo 7 anni), l'abbassamento medio anno fu di $2,0 \mathrm{~mm}$, mentre fra il 1942 e il 1968 si ebbe una media flessione anmua di 2,9 mm. Tutte le misure aventi come caposaldo Punta tlella. Dogana, risultano comprese entro una fascia l'incertezza superiore a $\pm 1 \mathrm{~cm}$.

Le misure mareografiche lanno mostrato un ineremento melio amnno del $l m m$ dell'ordine di $1,2 \mathrm{~mm}$, attribuito all'eustatismo.

Per quanto concerne il confronto fra i lati maregrafici di S. Maria. della Salute e di Trieste, si è osservata una difierenza di livello, con aumento deciso e progressivo per Venezia, a partire dal 1926 ca.

L'ablassamento non presenta caratteri di continuità né di linearità. Risultano sovmpposte evillenti ondulazioni. Va però sottolineato 
che i mareografi lagunari non hamno serie di osservazioni sufficientemente lunglie, per poter derlurre l'eventuale periolicitì delle ondulazioni sovrajproste e le loro caratteristiche fondamentali ( ${ }^{(1)}$; quindi il confronto con i risultati altimetrici è solo dli prima, grossolana ajorrossimazione.

In ogni modo, sulla base delle osservazioni altimetriche o mareografiche sembra di poter concludere the gli abbassamenti negli ultimi 40 anni (1929-1967), jer la zona di Venezia Centro - Marglera, siano stati rlell'orline di $2,7-3 \mathrm{~mm} / \mathrm{anno}$. Il movimento di abbassamento sembra cominciato tra il 1925 e il 1930 ed è tuttora in atto.

Questi risultati, per i motivi accennati, non sono esenti da riserve.

Tali riserve, naturalmente, si estendono a tutte le conclusioni e le illazioni, tratte partendo dai dati sojus accennati: tra esse, l'aver ritenuto valido un abbassamento complessivo del suolo veneziauo di cirea $20 \mathrm{~cm}$, a jartire del 1900 . I'acettazione di questo dato ba condotto a. ritenere clio le effettive acque alte - quelle attribuibili alle sole cause astronomiche e meteorologiche -, negli ultimi decenni non avrebbero subito incremento apprezzabile: il che porterchbe a concludere che l'aumentata frequenza dell'acqua alta sarcblo da attribuire al progressivo anmento del livello marino relativo, come somma del progressivo aumento intrinseco del livello del mare e del contemporaneo abbassamento del snolo di Venezia $\left({ }^{50}\right)$. Conclusione opinabile, naturalmente: da una somma di incertezze infatti non può soaturire un dato certo $\left(^{*}\right)$ E mia convinzione (sufferagata ila quanto esporrò piì avanti) che le cuuse del pì̀ frequente verificarsi delle acque alte non si limitano alle due qui citate.

8.2. - I risultati riassunti nel paragrafo precedente, sui quali gli stessi estensori non sono stati avari di riserve, han no provocato numerose contestazioni. C'i chi ne infirma l'attenrlibilità, partendo dalla constatazione che tre recenti livellazioni asseguerebbero al cajosaldo costituito dalla sommità dell'idrometro in ealle Loredan (Palazzo comunale di Venezia) un abbassamento, rappresentato da tre diversi valori:

$\left(^{*}\right)$ G. Supino $\left({ }^{51}\right)$ fa alcuni rilievi sul metodo seguito da IIosetti nelle valutazioni delle acque alte; fra l'altro, gli obietta di aver presn in ennsiderazione il livelln medio annuo dell'anno all'esame e non (come sarebbe stato più giusto) il livello marino " teorico $\%$, risultunte dall'ablassamentn del terreno e dall'aumento del mare. Ha, anehe nell'ipotesi della validiti dello determinazioni di Hosetti, a Supino non sembra si possa dedurre che non sia aumentata la frequenza delle acque alte eftettive negli ultimi clecenni; essa resterebhe comunque dimostrata, richiamando l'attenzinne sul fatto "che dal 1914 al 1943 (in 30 amni) vi sono state 4 aeque alte, mentre dal 1944 al 1969 , in 26 anni, ve ne snno state $11 \mathrm{~m}$. 


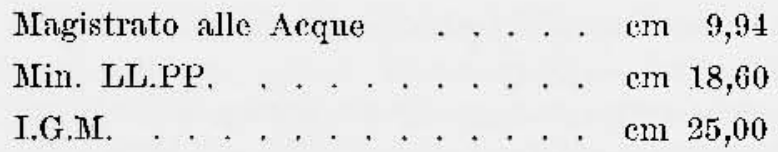

" Dette discordanze, che superano del doppio e del triplo la stessu prima grandezza bradisismica, sono incompatibili ed indieano pertanto l'esistenza di errori nelle singole livellazioni " (52). L'A. di questo giudizio, si dilunge in una particolareggiatia critica sui caposalili scelti per le diverse livellazioni e sulla loro più o meno mareata inidoneità, nonclié su spicente contraddizioni nei risultati (*).

A mio parere, la parte piǹ interessante del lavoro di O. Spagnuolo(53) i quella che riferisce l'abbassamento ad elementi costruttivi sicuramente collegati con il livello medio dell'acqun del mare "quali i ponti per il collegamento delle numerose isole di Venezia, i pozzi dell'acqua. potabile, i forti (...) ed ogni altra opera che abbia un dato di progetto (o di rilievo esecutivo) collegato con il livello del mare e rintracciabile presso l'Arehivio di Stato o nelle biblioteche veneziane" (l.c., p. 34). Iue ricerche compinte dkll'A., l'lanno condotito a rintracciare, presso archivi e biblioteche, i disegni e i progetil esecutivi, dai quali risulta, per esempio, ohe il Ponte della Paglia (la cui costruzione risale al 1360) aveva l'altezza del volto dal "Comm ilell'acqua" (livello medio del mare dell'epoca) a " piè $81 / 2$ " (l.c., p. 35), cioè a m 2,96 circa, clie si discosta poeo dalla misura attuale $(* *)$. Difierenze minime risultano per il Ponte di Rialto, il Ponte delle Guglie ed altri ponti. Stessa conchusione per altre opere, antiche di secoli, come il Forte di Sant'Andrea, le conche di navigazione (***), la Villa della Naleontenta,

(*) Naturalmente, io non posso entrare nel merito di queste critiche. Osservo solo che, per quanto concerne il ponte sul Crevada (nei pressi di Conegliano), la spalletta portante il caposaldo, alla fine di Novembre 1972, appariva restaurata. Noto inoltre che il caposaldo della livellazione dell I.G.31. c̀ a circa 500 in dal Crevada, a $\mathrm{m}$ 0,50 dallo spigolo nord-est del fabbricato "Ristorante Nazioni Unite". Sottolineo che questo caposaldo è ai margini inmediati della Strada Nazionale n. 13, soggetta a traffico intensissimo, in gran parte pesante.

(**) Sparnuolo traduce piedi veneti 8,5 in $\mathrm{m} \mathrm{2,80}$; il che fa pensare che egli abbia ritenuto il picde veneto inferiore alla misura äi $m 0,348$ cirea, quale viene comunemente riportata.

(***) A proposito delle antiche conche di navigazione fra bacino lagunare e eanali navigabili di terraferma, Spagnuolo ne sottolinea il regolare funzionamento: "Dopo quattrocento anni ui vita, queste imponenti opere idrauliche sono tuttora perfettamente funzionanti " (1.c., p. 49). 
i pozzi di raccolta dell'acqua piovana (di Campo San Trovaso, di Pellestrina, ecc.) .... (*).

Nalgrato tutito, ritengo che un giuoco di $10-15 \mathrm{~cm}$ possa intercorrere fra l'antico e l'attuale livello del mare; sebbene, una parte rlello searto - con riferimento ai manufatti - sia senz'altro da imputare all'inevitabile avvallamento di opere plurisecolari, quali i ponti, i forti, le colonne, certi edifici, ecc. destinati ad afiossarsi - chi piu chi meno nel terreno che li sopporta.

Altri studiosi, pur non negando l'abbassamento in atto, ne contestano l'entità ufficialmente ammessa, anche perché alcuni cedimenti locali, flovuti allo scavo di canali profondi (come il crollo di parte del forte di Sant'Andrea), o al erosione o ad altre canse aceidentali " vengono spesso fatti passare per abbassamento di tutio il bacino lagunare " [Pisenti e Rosa Salva $\left({ }^{53}\right)$, ]. 10]. Senza escludere fenomeni di abbassamento, gli $\Lambda A$. citati sostengono che questo va ridimensionato con valori molto più modesti; accettando quelli proposti, molti ponti plurisecolavi oggi risulterebbero intransitabili.

In ogni caso, se l'abbassamento del suolo lagunare non può essere negato (anche se la sua entità è probabilmente inferiore a quella proposta da aleuni esperti), un fatto, a mio parere, va fortemente sottolineato: l'abbassamento in questione tende ad aumentare verso Chioggia, per raggiungere valori eccezionali a Porto Corsini. Nel 1967, nella prefazione al mio lavoro sui fenomeni di anormale abbassamento verificatisi nel Delta Palano, osservavo: "Non va dimenticato che, limitrofe alla zona soggetta ad un abbassamento che, in aleuni punti, ha superato $i$ 3 metri e mezzo in circa un decennio, si trovano città come Venezia e Ravenna, in cui - negli ultimi anni - il sottosuolo sta subendo flessioni che possiamo definire anormali "[ [(+0) l.e., p. 332]. E poiché l'abbassamento del Delta veniva - già dal 1958 - attribuito alle smodate estrazioni di acque metanifere dal sottosuolo, aggiungevo (l.c., p. 398): "IJ'elietto delle estrazioni, indiscusso nelle zone comunque interessate dai pozzi, non è detto non debba estendersi a distanza dai luoghi dove

(*) In particolare, in eui l'A. del citato lavoro richiauna l'attenzione, ò quello eonecrnerite le due grancli eolonne, che si innalzano nella Piazzetta II San Mareo al margine del bacino. Questo colonne, erette nel 1172, "sono sempre rimaste alla stessa quota di impostazione, come si può comprovare dall'esame delle antiche stampe rafiguranti il Bacino di San Miarco " (l.c., p. 57). Sembri che alcuni esperti abbiano inteso spicgare l'inalterata quota altimetrica che le colonne conservano da otto secoli eol "rifluire lel terreno sotto In mole dei fabbricati della Lilureria e del Palazzo Dueale n (l.e., p. 57). Spero si tratti solo li una battuta polemica. 
è avvenula - o tuttora avviene - l'estrazione. Non va dimenticato che le flessioni determinate a Long Beach, in conseguenza dell'estrazione del petrolio, si sono sensibilmente estese in zone limitrofe, fino ad interessare la zona di Los Angeles, a eirea $40 \mathrm{~km}$ di distanza (...). A circa $16 \mathrm{~km}$ dalla zona di massima flessione (Ca Vendramin), l'abbassamento osservato dal $195 \mathrm{~L}$ al 1963 è stato di cirea $35 \mathrm{~cm}$, avendo raggiunto il valore di ben $20 \mathrm{~cm}$ dłl 1958 al 1963 , in soli sinque anni, dei quali tre dopo la sospensione degli emungimenti. Si tenga presente ele $15 \mathrm{~km}$ più a Nord si trova Chioggia, mentre Venezia i a circa $35 \mathrm{~km}$ dı Porto Oaleri... . . Concludevo che, se quelle correlazioni crano ritenute azzardate, sarei stato ben felice di trovarmi in torto. Questi concetti ribadivo nel $1970\left({ }^{(17}\right)$.

A proposito dell'abbassamento crescente da Venezia a Chioggia a Porto Caleri, qualche amno dopo la pubblicazione delle considerazioni sopma riportate, un esperto seriveva [( $\left.{ }^{(4)}\right)$ p. 253]: "Il fatto che Chiogria si sia abbassitł più di Marglier e Venezia, e che Porto Corsini presenti dei movimenti ancora più forti, farebbe pensare che almeno uno degli epicentri dell'abbissamento sia situato nella zona del Delta Parano. L'abbassumento del Delta del resto è gia stato provato, prescindendo dai dati mareografici, mediante livellazione di precisione $n$.

Ii ciò a conferma di quanto da me ripetutamente affermato in precedenza, fra la generale indifferenza o denegazione $\left({ }^{47}\right)$.

A conclusione di queste brevi note, si può osservare che le opinioni sulla stabilitì del sottosuolo di Venezia e della sua laguna, sono le più disparate; e vanno d $₫$ quelle di chi nega recisamente ogni, anche minima, flessione, a quelle di chi sostiene abbłussamenti di un'entità tale, dı far pensare che Venezia scompaia sotto il livello del mare nel volgere di pochi anni (*).

\section{9. - Crescente frequenza delle " acqte hlte TAZIONE.}

9.1. - L'abbassimento, indubbiamente, è in atito: forse si esıgera, in un senso e nell'altro, nellit valutazione della sua entiti.

(*) P. es., vi è chi afferma che lo sprofondanento (per eanse tettonjehe) è stato, negli ultimi 1.800 .000 anni, di $1 \mathrm{~mm} /$ anno, al quale andrebbe sovrapjosto quello derli ultimi lustri, dell'ordine di 5-7 mm/anno (conseguente all'impoverimento delle falde acquiftere); se si agrinuge l'innalamento del livello del mare per eustatismo, se ne sovrebbe concludere che, all'insaputa generale, attualmente Venezia è allo stato di grallegrianento. 


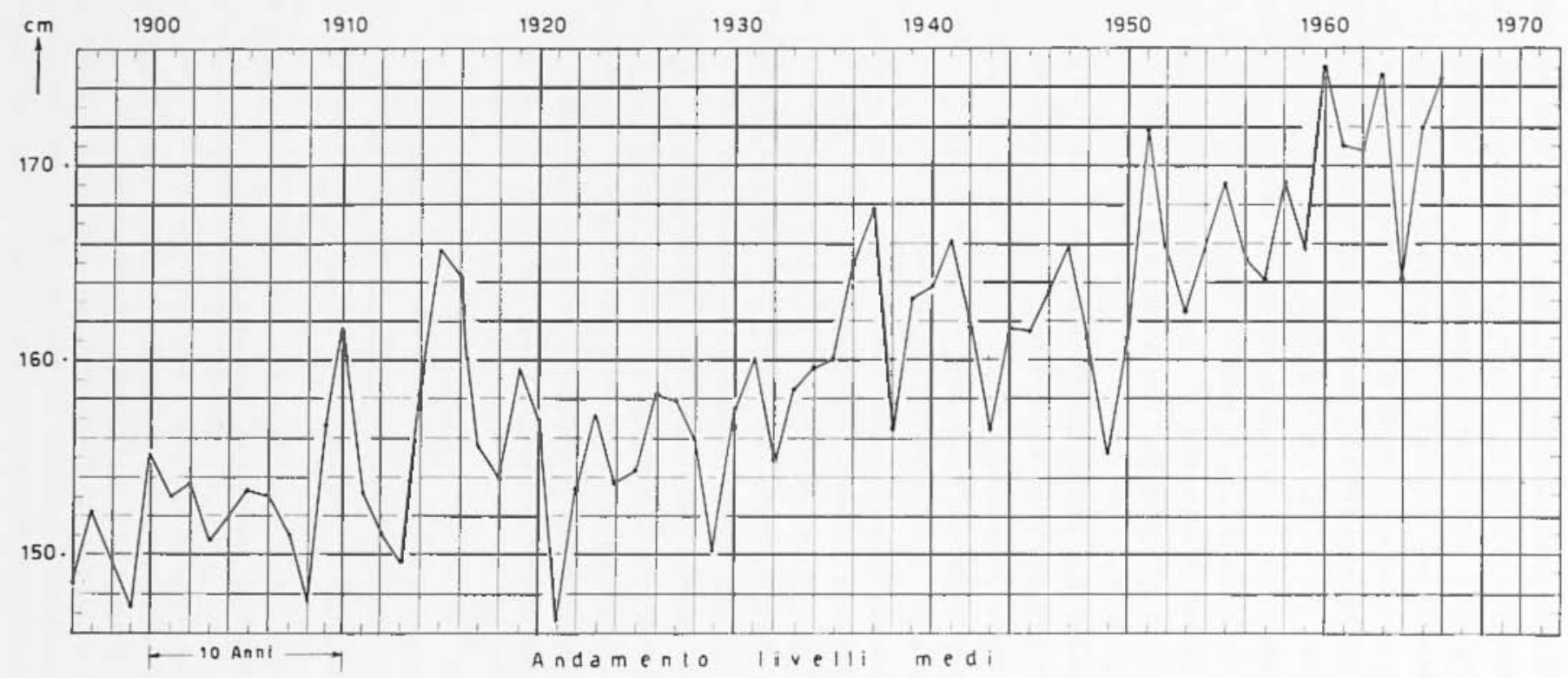

Fig. 19 - Andamento livelli medi (Venezia, P’unta della Salute).

Fig. 19 - Trend of mean levels (Venice, Punta della Salute). 
Di effettivo, purtroppo, e'e l'allamante aumento dei giormi ti "acqua alta" a Venezia. Per limitarei agli ultimi decemi, le mares superiori ai $110 \mathrm{~cm}$, passano dalle 2 acque alte del periodo $1916-1926$, alle 8 del 1927-1936, alle 3 tel 1937-1946, alle 10 del decennio 19-7 1956 , fino alle 29 del decennio $1957-1966$, tendendo ad ammentare neggli anni suecessivi. Se poi si prendono in eomsiderazione i livelli superiori ai $70 \mathrm{~cm}$, si passa dai 161 chi de1 $1964-66$, ai 197 del biennio $1966-138$, fino alla frequenza massima mai ragginuta di 295 del biennio 1968-1970 (I'isenti, Rosa Salva, 1.c., p]. 9, 10).

Richiamo ora lattenzione sopra un particolare, al quale attribuiseo una certa importmza. In generale, i grafici relativi a livelli marini vengono perequati seconflo curve continue. $\mathbf{E}$ mio parere ele, in quest'ordine d'indagini, tale procerlura funisea eol mascherare brusche variagioni nell'andamento del fenomeno indagato. Gli americani Domn e Shaw, nel lavoro citato $\left({ }^{36}\right)$, preferiseono mediare lumgo tratti rettilinei, appliando il metorlo dei minimi qualrati. Un tale procedimento, applicafo p. as. ai tratti $1935-1950$ e 195̆-1969 dol diagramma dei livelli medi registrati a Punta della Salute (fig. 19), rivelerebbe una soluzione di continuita nella tendenza del fenomeno delle acque alte, intorno all'anno 1950 .

Del resto, questa soluzione di continuita nell'andamento del $l m m$ intorno al 1950, si osserva ancle in altri porti della costa occidentale del golfo di Venezia. Si noti p. es. l'andamento del $l m m$ annuo di Porto Corsini, a Sind del Delta, quale risulta dai chati l'osservazione per il periodo 1934-1967 [(14) p. 246]. Essi sono riportati in fig. 20. Si nota cliaumente il salto buzses della tendenza all'innalzamento, che si verifica appounto intormo al 1950 .

Ma una discontinuita intomo a quell'epoca risulta anche in altri studi. In particolare, l'Ing. Paolo Pirazgoli (js) ha preso come bise dalla sua ricerea il $l m m$ anmuo elabouto dall'Ufficio Idrografico del Iagistrato alle Acque di Venezia, sulla scorta delle registrazioni mareografiche ottemute nel periodo 1871-1968 flal 1871 al 1906 tal marografo di Campo Santo Stefano, e dal 1900 nella stazione di P'unta tella Salute). I dati appaiono alquanto dispersi, ancle se nell'issieme rivelano una tendenza all'numento (fig. 21). Pirazoli, allo seopo di eliminare sovrapposizioni attribuibili a condizioni meteorologiche particolari, at enori 0 al altre cause accillentali, eftettuo alcuni filtruggi, sostituento ad ogni valore anmule del $7 \mathrm{~m} m$ " la media di un certo mumero di valori consecutivi, di cui il valore dato costituisce l'elemento centralen. I risultati sono riassunti nelle prime due figne del lavoro citato. L'A. 
nota che «in complesso si puo osservare the le curve delle metie correnti presentano le seguenti caratteristiche commui: un periodo con piccole oscillazioni intorno al un valore pressoché costante flino a

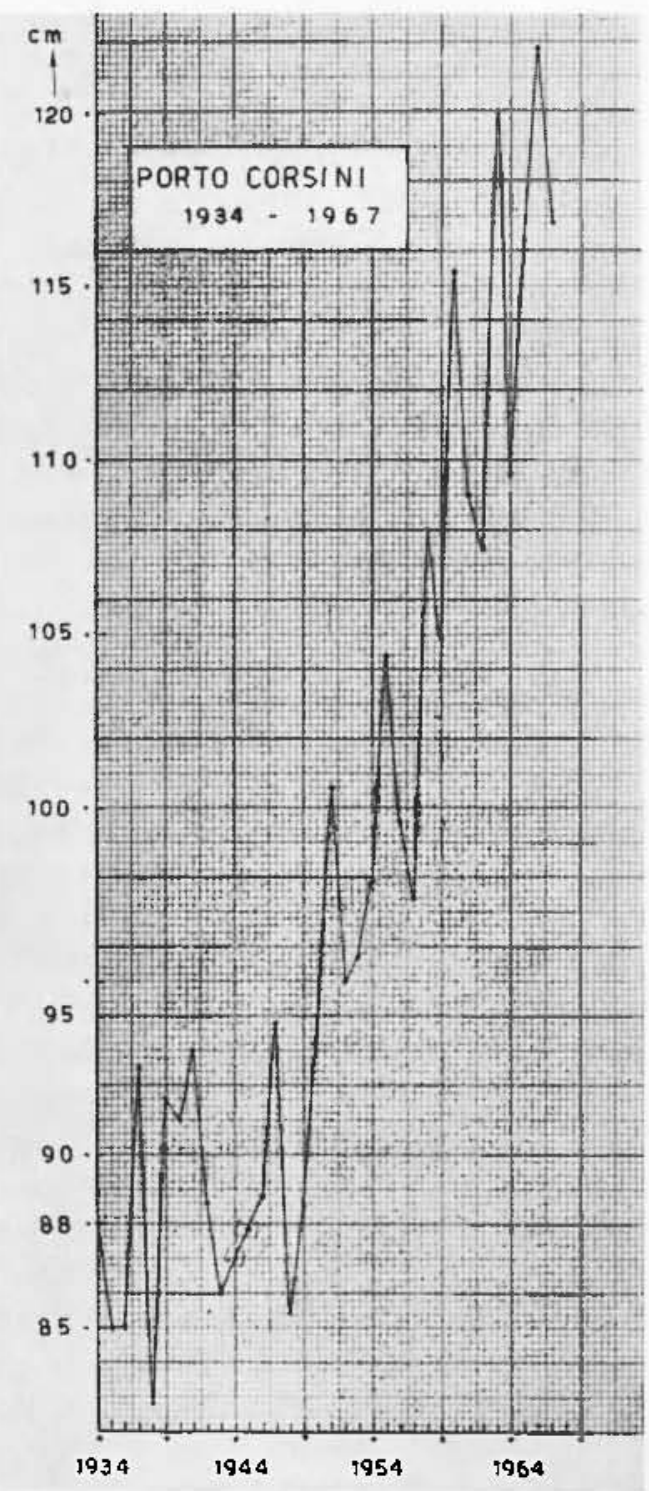

Fig. 20 - Anlamento livelli medi del mare a Porto Corsini.

Fig. 20 - Trent of mean sea levels at l'orto Corsini. 
cirea il 1890); un periodo dli erescita pressoché lineare tra il 1890 erl il 1910 cirea; un periodo con oseillazioni importanti intorno ad un valore tendenzialmente creseente fra il 1910 ed il 1950 eirca; un nuovo periodo di creseita pressoché lineare dal 1950 in poi n (1.e., p. 3). Le curve ottenute furono quindi sostitnite dia ma linea, consistente in tratti di retta o di parabola, ealcolando i parametri dei singoli tratti con il metodo dei minimi quadrati (previa seelta dei punti di discontinuità).

Si noteri che, in tutti i cusi, il 1950 costituisee un punto di discontinuitì. Anche l'oscillazione sovrapposta (fig. 21) presenta una forte ripresa del livello marino verso il 1926 - che Pirazzoli attribuisce al

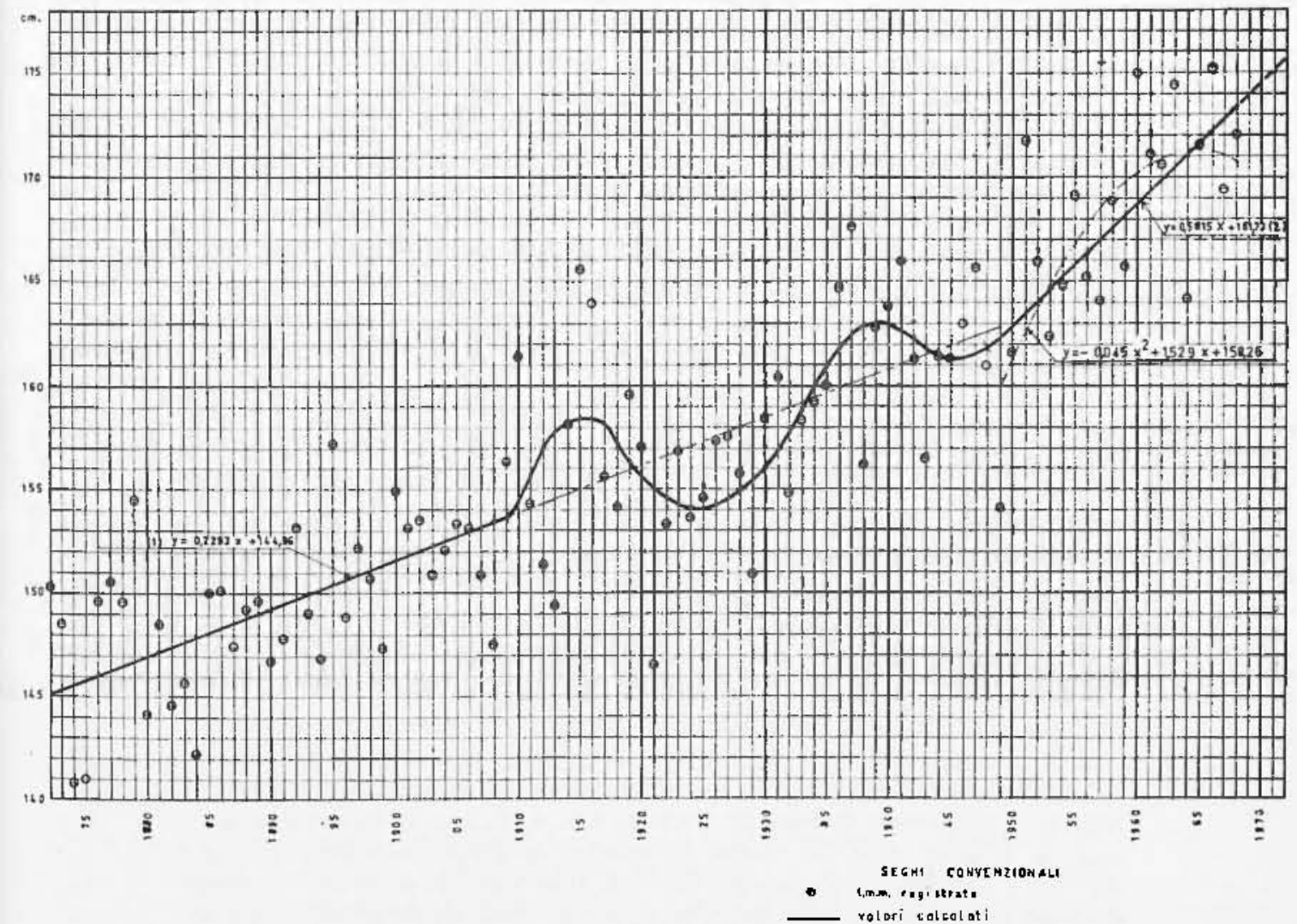

Fig. 2 - Tratti di rette e tratti di curve calcolate ta P'. Pirazzoli ( $\left.{ }^{(5)}\right)$ sulla buse tlej $1, \mathrm{~m} . \mathrm{m}$. registrati a Venezia.

Fig. 21 - Segments of straiglt and eurved lines, caledated by P. Pirazzoli (st) on the basis of the mean sca level recorden in Venice. 
rilancio industriale di Porto Marghera -; ma la vera discontinuiti, nettamente marcuta, è quella intorno al 1950 .

Ricordiamo ehe, a partire da quella data, erano in atto gli anormali abbassamenti del suolo, provocati nel Delta padano dalle disordinate estrazioni di acque metanifere e che, inoltre, nel Novembre 1951 si able ma delle maggiori piene del Po degli ultimi secoli.

Ci solfermeremo ampiamente su questi eventi, ai quali è intimamente legata l'allarmante frequenza delle aeque alte nelle lagune venete $(*)$.

\section{2. - Epirogenesi e insubbiamento nell'allo Adriatico. Abbassamento dellu costa oceidentale e sue consoguenze.}

Come si è sopra ricordato, nell'antumno del 1951, flopo un lungo periodo di piena, il Po ruppe gli argini nei pressi di Oechiobello e dilago in una vasta zona del Delta, ruggiungendo il mare sopra un fronte esteso. Nel lungo periodo in eni durò la piena e la successiva inondazione (piena. che naturalmente si verifico anche negli altri fiumi che sfoeiano nell'alto Adriatico), il materiale di torbida e di erosione traseinato in mare fu di certo enorme, quale soltanto può forse verifiearsi in decenni di normale confluire dei fiumi allia foce.

Ciò ha certamente contribuito a rendere più pronunciata la diflerenza fra le medio profonditi del basso e dell'alto Arlriatico o ad accenturu'e, in modo brusco, la naturale temlenza, intesa a far confluire, verso valori comuni, i periodi delle onde forzate, a delle onde libere del mare, con conseguente aumento delle ampiezze delle "ucque alte", a paritis di perturbazioni meteorologiehe.

(*) Dalie serie di livelli marini di alcuni porti italiani furono tentate delle analisi periodali. S. Polli applicò la cimanalisi ad una serie cinquanten. nale di Trieste (1890-1942) o ad una settantennale di Venezia (1872-1941) $\left(^{55}\right)$. Ottenne componenti di anni $22 ; 11,3 ; 8 ; 5,5 ; 4 ; 3 ; 2$. L'onda di 22 anni comme a luasi tutte le analisi climatiche, in mecelenza fatte da altri studiosi --, eorrisponde al doppio del ciclo delle macehie soluri ed è ritenuta da molti autori quale ciclo fondamentale; da esso seguirebbero i cicli armonici di $11 \cdot 7,5 \cdot 5,6$ anni.

Una analisi spettrale dei dati relativi a $l m m$ di Porto Corsini, Vernezia, Genova a Trieste, fu sucessivanente cfict tuata -... con il metodo di Fourier (la Caputo ed al. $\left(^{(58)}\right.$. I perioli otlenuti per tutti i mareografi, furono di anni: $20.0: 12.5: 8.3: 6.7: 4.0: 3.4$. 
FRANE E FENOMEN! DI DILAVAMENTO

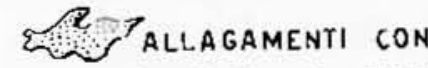

ACCUMULI ALLUVIONALI

ALLAGAMENTI FUOR!

OEGLI ALVEI ABITUALI

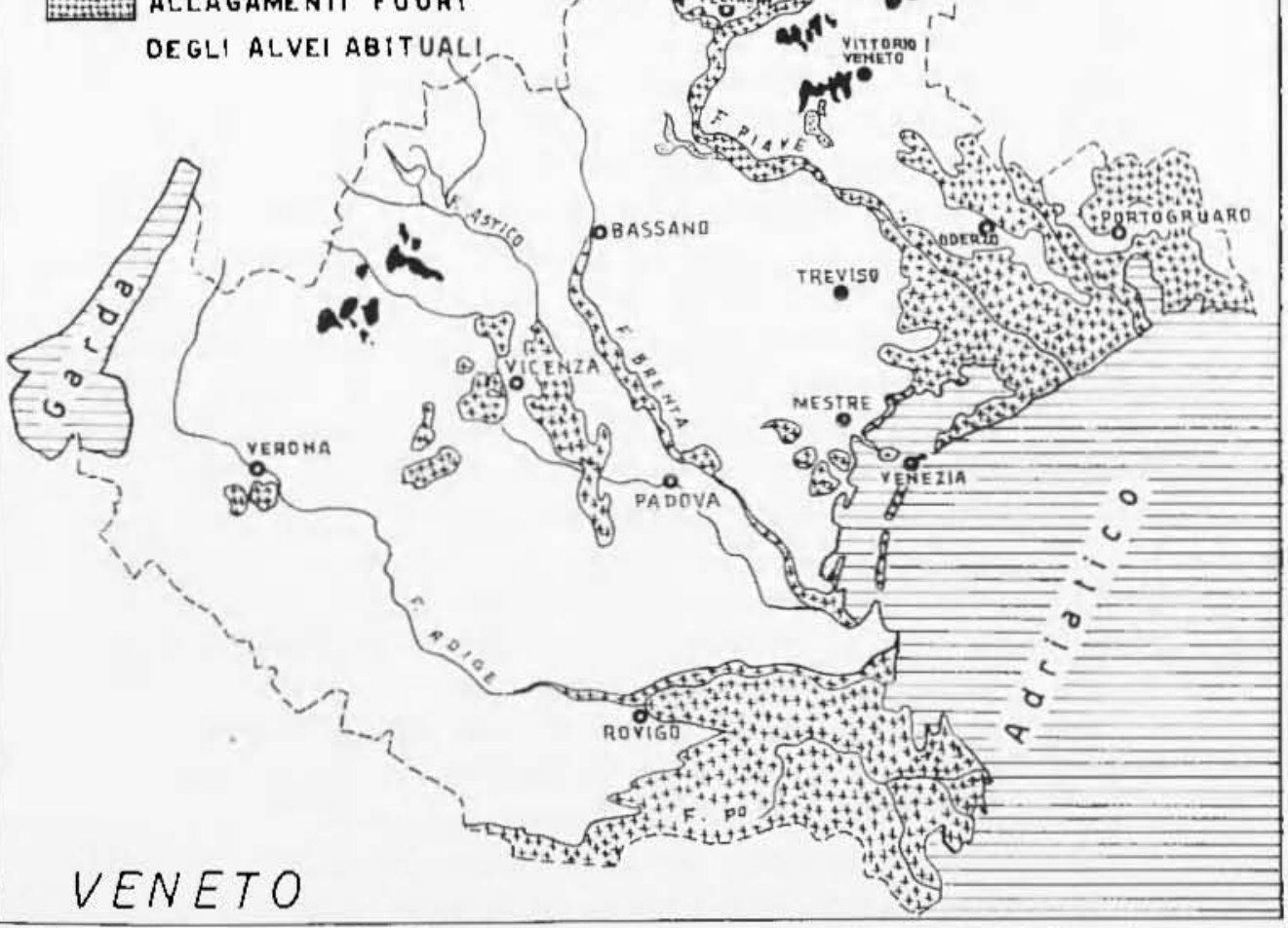

Fig. 22 - Allagamenti fuori dogli alvei ed altri femomeni associati all allu. vinne dei primi ali Novembre 1966, limitatamente al Vencto. (Dalla "Carta dell'alluvione del Novembre 1966 nel Veneto e nel Trentino-Alto Adigen, a cura dell'Jstituto di Geografia dell Cniversiti di Padova e di altri Enti.)

Fig. 22 - Flood and other phenomena to the alluvion associated of the Venetian countrysicle, early in November 1966. (From the Map edited by Geographical Institute of the University, Padua, and hy other Depart. monts.) 
Ia, riandando più addietro nel tempo, troviamo moltha chiara coincidenza fo'a srandi alluvioni e successivo incremento nelle tacque alte $\%$. Il 1926, il 1927 a il 1928 furouo (come lo fu il 195̆L) anni di grandi piene di parechi fiumi dell'Ttalia settentrionale $\left[\left({ }^{57}\right)\right.$ tab. I]; in particolave, le magriori piene tel Po dell'ultimo secolo, oltre a quella eitata lel 1951, furono appyunto quelle del Yasgoio e Fovembre 1926 [(57) p. 163]. A partire dal 1925 la frequenza delle acque alte si aceentuo bruscamente provocando il contemporaneo apparente aumento del livallo medio. A Venezia $\left[\left({ }^{8}\right)\right.$ p. 7], le maree nuguali o superiori a $\mathrm{m} 1,10 \mathrm{sul} 7 \mathrm{~m} \mathrm{~m}$, che nei 43 anni fra il 1884 e il 1926 erano state solo 3 , nei 12 anni successivi fra il 1926 e il 1938 , balzaromo bruscamente a 10 . Invocare alterazioni locali per insediamenti industriali a Harghera, mi sembra eccessivo; sia perchó l'inizio di quegli insediamenti fu modesto - e tale si conservò per molti anni (*) —, sia perché il fenomeno delle arque alte si acentuó contemporaneamente altrove, da Venezia a Porto Corsini. I mia convinzione che la causa principale va ricercata nell'improvviso squilibrio, verifientosi nel giuoso fra eustatismo a flessione tettonica (questa in molto minor misura) da un lato e l'azione del lento insabbiamento per anormali apporti di torbida e di prodotti di erosione dall'altro, nettamente a favore di quest'ultima; col contemporaneo rinforzo tlell'interazione fra atmosfera el iduosfera (dovnta in parte alla lievitazione delle acque per diminuita salinita), a parità di perturbazioni meteorologiehe e eon l'necentuarsi dell'efletto di mi ai nn. 3,5,6. La scomparsa delle aeque alte nel decennio 1938-1947 (e il contemporaneo apparente vistugno del $l \mathrm{~m} \mathrm{~m}$ ), sta a sisnificare che lo squilibrio sopra accennato si andava attenuando con il ritorno dei normali aj)porti di torbiala, la ripresa tlella salinita e il persistente enstatismo.

Ya il naturale equilibrio torob al essere infranto nel 1951, con l'immane alluvione del Novembre di quell'anno e il consegruente improvviso incremento tell'insabbiamento nell'alto Adriatico.

f) qui ritengo opportuno riprendere l'argomento relativo alla lotta fra terra ca acqua nella fossa padana, già in parte trattato al n. 2.

È noto che fra una glaciazione e l'altma, la depressione padana passò ripetutamente dal regime continentale a quello marino.

$\left({ }^{*}\right)$ a Marghera nacque e si realizzò come opera limitata. P'iǹ tardi, in contraddizione al buon senso di Volpi, la zona fu aperta a nuovi spazi " $\left[\left({ }^{59}\right)\right.$, p. 98]. I lavori, iniziati nel 1919 , furono ultimati nel $193 \overline{5}$. Si noti clie, nel 1922, gli stabilimenti erano 16; mentre nel 1955, quando Ginseppe Volpi era da tempo scomparso, eramo saliti a 180 ! 
Va comunque rilevato - come scrive P. Leonardi - "che ad ogni ritorno nella depressione pulana it mave divenira man mano meno profondo e meno esteso, perché, ad onta dellabbassamento progressivo dovuto al costipamento dei materiali sedimentari e - nella zona costiera - al bratisismo positivo tuttora in atto, nei periodi di emersione le fumane divaganti sulla pianura deponevano gran quantitì di materiali alluvionali, che pian piano andwvano colmando il gollo padanon $\left({ }^{60}\right)$.

Questo fenomeno ali riempimento slell'alto Arliatico is andato aceentuandosi negli ultimi secoli, in seguito ai lavori di regolamento ed arginatura dei fiumi. Così, il delta slel Po dal X I.I al X VII secolo avanzò annualmente di ciren $25 \mathrm{~m}\left({ }^{61}\right)$, mentre dal 1600 al 1804 crebbe in ragione di eirca $73 \mathrm{~m}$ all'anno: "differenza dovuta alle arginature artificiali, che mantennero alla corrente la maggior parte del materiale sospeso, che prima voniva in gran copia disperso lungo il corso del fiume" ("92). Sempre on riferimento al Po, Harinelli $\left({ }^{63}\right)$ calcolib che l'acerescimento medio del delta nel settantennio 1893-1893, sia stato dell'ordine di $0,762 \mathrm{kmq}$ all'snno.

(1) stata anche fatta una stima del moterisle solido in sospensione, trasportato dal Po in mare, ed ivi dalle correnti variamente distribuito sul fondo. Tale quantiti varia natumente a seconda del clina e delle stagioni. Così, nel decennio (non molto piovoso) 1914-1922, la quantitì̀ di materia trasportata in sospensione slal Po risulta di circa 17 milioni c mezzo di me allanno (0.), mentre per il settantennio prima citato, Harinelli valuta che il ceposito medio annuo fu di circa 29 milioni di mo. Alla parte di materiale trasportata in sospensione, va peró aggiunta quella condotta per triscinamento, che costituisce circa il $10 \%$ selle materie solikle in sospensione ("*).

In ogni caso, ammesso che annulmente il Fo trascini a mare cirea 30 milioni di me di materiale solido minuto, un breve calcolo prova

(*) Giantotti [(-1) 1.c., p. 3], a proposito del trasporto anno di materialc solido da parte del $P o$, riporta che o il Lombarlini, sulla base della misura del protendimento del letto del Po, venne alla conclusione che il volume di materic convogliate chal fiume, ascencleva in mella a 25 milioni di me in cifra tonda " (Lombardini, Sul sisteme idraulieo det Po, MLilano - 1843).

Non v'è chlolyio, però, che il trasporto di materiale solito in sospensione tloveva essere, in realth, nethancente superiore al valore riportato: infatiti, la maggior parte alcl materiale solito sospeso - specialmente quello piu sottile vicne traspordato lontano dalla fuce e uon contribuisce al "protendimento" tlel delta. 
che esso è sufficiente a coprire, con uno strato tlello spessore di $u \mathrm{~cm}$, un'aren dellordine di $3000 \mathrm{kmq}$ (cios', all'inciren, una superficie tli $50 \mathrm{~km} \times 60 \mathrm{~km})$ - anche se, naturalmente, soprattutto a causa delle correnti, la distribuzione non avviene in morlo uniforme.

Ma il P'o non ¿̀ il solo fiume - anche se il maggiore - clic immette le sue arque nell'alto Alriatico. Particolarmente i fiumi veneti, dall'Adige all'Isonzo, portano il loro complessivo contributo di torbida nel golfo di Venezia. L'Adige, p. es., nel 1933 - seconto i dati pubblicati dall'Uificio Idrografico del Magistrato alle Aeque - ha trasportato in sospensione (come fu rieavato da ancurate misure di torbida, eseguite a Boara Pisani) tomellate 1.096 .800 ali materiale solido (65). In un anno, quindi, l'Alige porta a mare - in una valutazione approssimativa - materiale sciolto capace di coprire un'area di ea. $100 \mathrm{kmq}$ per lo spessore di $1 \mathrm{~cm}$.

Ira non è tutto. Nelle cpoche tli piena, l'apporto di materiale è enormemente maggiore. $P$. es., la Loira in piena conduce una massa. d'acqua valutata 312 volte quella dello scolo normale. Tale rapjorto diminuise sensibilmente se un fiume attraversa zone boscose: per il corso del Reno, al di sopra del lago di Costanza, lo stesso rapporto è infatti di $70: 1$ [De Marchi $\left.{ }^{62}\right)$, 1.c., p. 2-4i]. In ogni morlo, resta sempre molto elevato. Durante una piena, un fiume può quindi trasportare a mare materiale sciolto ehe, normalmente, suole immettere in decemi.

Il sollevamento del fondo dell'alto Artriatico, per immissione di materiali solili minuti da parte dei fumi che vi sfociano, supera quindi largamente le variazioni di livello provoeate ta eustatismo. A parità di ause perturbanti, tende quindi lentamente ad aumentare l'ampiezza. delle oscillazioni che interessano l'alto Adriatico; aumento che si accentua bruscamente, in concomitanza di improvvise ondate di piena (18791882, 1926-1928, dal 1951 in poi). Ma il sollevamento tlel fondo marino, causa una variazione ancora più insidiosa: l'aumento del periodo proprio tel tratto di mare interessato da oscillazioni libere, specie di quelle riguardanti lat to Adriatico. Si sa che, a parità di altre condizioni, tale periorlo $i$, in via approssimativa, inversamente proporzionale al quadrato della profondità; ed ammenta quindi al diminuire dli questa. Poiché, p. es., l'onda libera di $11,8^{n}$ è prossima alla marea semidiurna, anche un lievissimo aumento del suo periorlo - essento in zona di risonanza — può provocare sensibili aumenti d'ampiezza.

Dal 1951, però, altre due cause hanno contribuito all'accentuazione delle acque alte nelle lagune venete: $1^{\text {a }}$ ) Il piu frequente ripetersi delle 
ondate di piena nei fiumi veneti (*): Tagliamento e Livenza (Febb. e Nov. 1951, ()t. 1953, Dic. 1954, Nov. 1959 , Nov. 1961 e - della massima violenza - Sov, 1965 e Kov. 1966), Piave e Brenta (Nov. 1951, Ott. 1953, Ott. 1960, Nov. 1965 e - notevolissima - Nov. 1966), Adige - Bacehiglione (Nov. 1951, Ott. 1953, Ag. 1966 e - di particolare entiti - Sett. 1960 , Seft. 1965 e Nov. 1966) - Da piceoli, l.c., Tabella $I$ - La progressiva accentuazione e frequenz: dei fenomeni di piena (col conseguente, progressivo aumento del trasporto di materiale in mare), dal Servizio Ialografico del Magistrato alle Arque è attribuito "alla ricorrenza delle precipitazioni intense in periodi sempre piì vicini s [Piccoli ( $\left.{ }^{57}\right)$, l.c., ], 15i].

2") L“inizio, e il progressivo accentuarsi fino 19.1964 (ed oltre) dellat. flessione della zona del Delta, e quindi della prospicente fascia di fondo marino, con speciale riguardo al tratto Porto Corsini-Chioggia. Per i motivi esposti al n. 6, questo fatto ha creato le condizioni sempre jiì idonee per l'umplifienzione delle onte - libere o forzate - propagantisi verso la costa.

\section{3. - Consequenze delle improvvise variazioni di salinità nell'alto Adriatico.}

Consideriamo un altro as]etto delle condizioni fisiche lell'ddriatico settentrionale, legato all'anomale apporto di acque dolei: quello della salinità. İ noto ehe la salsedine nei mari interni è tanto minores quanto più ridotita è lar loro comunicazione coul l'Ocessio, quanto minore l'evaporazione e quanto maggiore la massa d'acqua tole portata dai fumi. Mentre, 1\%. es., la stalinitì del Nar Rosso è dell'orline di 45\%, quella del Paltico ì, in media, di $15 \%$ potendo scendere nei golfi di Botnia e di Finlandis nettamente al disotto del $10^{\circ} \%$.

(*) A projosito dell'azione di riempimento del golfo di Venezia, da parte tei fiumi veneti in piena (e quindi dell'acentuata predisposizione ihro. dinamica alle "acelue alte") rammento che, sulla seorta dei dati forniti da

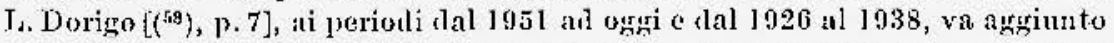
un primo gruppo di " acque alte $n$ fra il 1879 e il 1883 . Wibbene, nel 1879 si chlse una delle magriori piene del Po del secolo scorso [Ciandotti $\left({ }^{61}\right)$, l.c., p. 7], mentre si rese tristemente celelre l'autunno alel 1882 , per le piene, particolarmente funeste, di tutti i fiumi veneti, a cominciare dall Adige [P'iceoli, (5i) 1.c., pp. 156-157]. 
I periodi di piena portano quindi con sí altre ronseguenze sulle caratteristiche dell'alto Arriatico. L'enorme quantiti di acou rlolce che i fimmi rigonfi immettono nel golfo di Venezia dhrante le grandi piogge, alterano profondamente la salinità delle arouc del mare. Ta rliminuzione rlella salinità porta con sé, fra l'altro, la fliminuzione della densita, della viscositò molecolare e della tensione superficiale (60), ciò rente le acọne piǹ leggere e più fluide (più molbili). La vastità đel fenomeno finisce per interessare tutto l'alto Arliatico, dove appunto la salinità - dopo le grandi piene - subisce flessioni cospicne $\left({ }^{67}\right)$, A pariti di sollecitazioni da parte dell'atmosfera (variazioni di pressione in $\mathrm{mm}$ di mercurio), il rapporto mercurio/acqua non ì piǹ di 13,2 circa, come in caso di normale salinita, ma tende a 13,5. La variazione statica ri livello tende at aumentare, sia pure lievissimamente; e l'aumento diviene senz'altro apprezzabile in compo dinamico, quando si tende verso le conslizioni di risonanza. In questi cusi (nei quali l'eftet to statico può essere moltiplicato per 10-100 volte), la densiti, la viscositi molecolare e la tensione superficiale in diminuzione possono agevolare aumenti di livello dell'ordine di qualche centimetro.

Ma nom ì tutto. Si sa che l'isostasi presuppone che per uniti d'area sopra una superficie convenzionale (superficic di compensazione) incomba una colonna verticale ti ugua. massa, in qualunque punto della superficie considerata. Pertanto, tale colonna avrà un'altezza diversa a. seconda della densita: quindi, sarà maggiore ove la materí è meno densa e più leggera. Ebbene, Pattullo $\left({ }^{38} \cdot 4^{0}\right)$ dimostra che la parte più cospicua della deviazione del livello medio del mare è funzione di efletti isostatici, risultanti fra pressione rel mare e cumbiamenti di densiti (o volumetrici). Tali efletti mantengono nua pressione costante sulla superficie di compensazione (che può essere quella del fondo). In collaborazione con E. Lisitzin, Io stesso Pattullo provi che, dai risultati rlei mareogrammi registrati durante l' Amno Geofisico Internazionale ", la variazione stagionale del livello marino è principalmente isostatica $\left(^{(68)}\right.$, almeno nella maggior parte rel Pacifico.

Come è stato gì ricorflato, monceurata ricerea sulle variazioni del livello marino nelle zone atlantiche d'Islanda e di Bermula fu condotta da Shaw e fonn (n, 7), su rati abbraceianti il periorlo 1958 1962. Fra gli altri risultati, di notevole interesse quello relativo all'effetto isostatico, yisultante dall'effetto "steric" (legato alle variazioni nel volume specifico dellacqua) e da quello barometrico: l'efietto asteric " è di gran lunga preslominante (nei valoni positivi) nei mesi intomo all'Ottobre (Settembre-I)icembre), specie negli anni 1955-1957 \& 1961 
(uel Settembre 1955 avendo superato i $24 \mathrm{~cm}$ ), come dol resto appare anche dalle medie mensili dell'intero periodo, riportate nella fig. 16.

Cosa sucesle nell:alto Arlriatico durante e ilopo le grandi alluvioni? Lo "steric effect ", particolarmente associato alla forte flessione nei valori lella saliniti, deve assumere notevoli proporzioni e tradursi in un innalzamento ali livello di parechi centimetri. Una zona di transizione e compensazione, a giaciturn subrerticale, viene probabilmente a formarsi - in questi intervalli anomali - verso il medio Adriatico, fra Pesaro e Ancona, dove - anche in epoche normali - la salinità (per es. nell'acqua costiera di fronte a Fano) è più bassa in direzione NW el aumenta verso SE, come conseguenza dell'aflusso di acque dolci dei flumi veneti e, in particolare, ilel $P\left({ }^{(69}\right)$.

Nei periodi di piena dei fiumi confuenti nell'alto Ndrintico, e in quelli inmediatanente successivi, l'calleggerimento "delle acque per diminuita salinith, può provocare sollevamenti del livello marino per efletto isostatico, nonché agevolare - a parita di altre condizioni fenomeni dinamici di amplificazione dei movimenti dell'acqua. Ciò spiega perché gli anni delle maggiori piene sono pure caratterizzati da una nuargior frequenza delle acque alte $\left(^{*}\right)$ e come questa sia in accentuazione negli ultimi decenni, in eui ì manifesta un'intensificazione delle precipitazioni atmosferiche.

Ciò che ì stato aletto in questo paragrafo puo, in parte, sembrare in contrasto con quanto ì es]osto al 11.9 .2 ; non va peró dimenticato che si tratta di acque "lievitate", particolarmente atte a subire e al esaltare gli effetti burometrici dinamici; la loro massa commoue è sempre quella dello strato liquido delle stagioni normali.

\section{0. - Coxolusioni.}

a) Da quanto è stato osservato al n. 5 , poiché il medio spessore del mure in corrispondenza della profonda conca meridionale adriatica, può ritenersi costante - data l'estensione nettamente maggiore del bacino e il minor apporto di materiali di torbida in esso convogliati dai piceoli

(*) L'effetto opposto, naturalmente, si verifiea in easo di salinitù ereseente, per seursa piovosita e magre Anviali. T'aumento della densita puo essere tale da determinare a un effettiva contrazione dol volumu del mare, rivelabile anche attraverso la permanenza di livelli marini abbassati rispetto alla norma " [Nosetti $\left({ }^{67}\right), 1$. 4]. 
finmi che vi sfociano — nei confronti del medio spessore dell'alto Ariatico, in fase di assottigliamento, ne consegue (a parità di altre condizioni) un sia pur lento, ma continuo, aumento relativo dell'ampieza dei lenti movimenti del mare - associati a onde forzate o libere - nei pressi delle coste venete.

b) Xa la diminuzione della profondità di un bacino (o di una sua purte) fletermina unaltra consegnenza: it progressito aumento coeteris paribus - del periodo delle oseillazioni libere del bacino stesso.

Come si i detto, la teoria valuta il periodo inversamente proporzionale alla raflice quarlata della profourlita. Una diminuzione della profondita meslia dell'alto Arlriatico, comporta pertanto nu aumento rlel periodo delle oscillazioni proprie di detto mare (*), tanto più apprezzabile se loscillazione propria di $23^{\text {h }}$ sia da attribuire - come sostiene Oddone (") - ad movimento libero dell'alto Adriatico, come golfo aperto sulta vasta conca meridionale.

In ogni caso, l'aumento del periodo delle oscillazioni libere provoca un lentissimo, ma inesorabile, avvicinamento della [10] alle condizioni di risonama, specie per quanto si riferisce alle mares diurne e semirlinrue (**).

$\left.{ }^{*}\right)$ Se il periocho dell'oscillazione libera uninodale del tratto di mare fra lo eoste venete e la linea Tremiti-lelagrosa c̀ li $11, \mathrm{gh}^{\mathrm{h}}$ per ma profonditi metlia di $60 \mathrm{~m}$, diminnembo la profondita media di $2 \mathrm{~m}$, il petiodo dell'uninodale diviene di $12^{\mathrm{h}}$.

(**) A proposito della propagazione delle onde attraverso lalto Adriatieo, ei sautebo da tener eonto dell'azione dell'attrito sul fondo. Essa però salvo in canali stretti a per periodi molto lunghi, - es estremamente dobole.

Con riferimento alla [9] - o alla [10] - se consideriamo le he corrispondenti alle oseillazioni proprie, in easo di attrito esse non saramo più immagiuarie pure, ma avranno nna patte reale, che sarit sempte negativa, perehé l'onergia andrà decrestendo. Je ralie in $i$, saranno ancora immaginarie coningate due a due, ma non saranno piu a tue a due nguali e ili segno contrario.

Vediamone lo conseguenze. Tel raso di ostillazioni forzate, abbiamo $q_{r}=\varepsilon_{r} e^{\lambda_{t}}$, che d una soluzione particolare; mentre la soluzioue generale delle

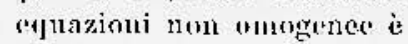

$$
q_{r}:=\varepsilon_{r} e^{\lambda \cdot i}-\sum \Lambda_{f} a_{r j} e^{\lambda} y^{t}
$$

Ite $A$; lipendono ialle eondizioni iniziali. Nel caso delle maree, questi termini spariseono rapidanente. Infatti, avende is una parte reale negativa, edjt vih costantemento decresceurlo, per estinguersi completanente: duinte levolversi dello marec, il movimento devulo alle oscillazioni lilere deve essere 
Del resto, e gia stato osservato alto volte, in bucini soggetti ad oscillazioni forzate, la tenkenza all'nnificazione dei periodi di dette onde - 0 di aleme fra esse - com i periodi delle oscillazioni libere, di cui il bacino ì capuce: uninodale, binodale, . . o di tahma fra queste. Nel 1910, Odilone $\left({ }^{70}\right)$ ha provato sperimentalinente come un bacino può alterare la sua configurazione, funo a pervenire ad un periodo proprio (lell'orline di quello associato alla forza perturbatrice.

Per quanto riguarda l'Ariatico, non ce certo easuale che luminodale di $23^{\text {n }}$ ch. sia prossima alla marea diurna e l'onda libera di $11,8^{\text {h }}$ ea. sia dell'ordine della matea semidiurna.

Nel suo audamento naturale, tale lavorio è indubbiamente lentissimo. it vero che il periodo del bacino pus essere allungato dal lento sollevamento del fondo, per ajporti di torbida, ma tale allungamento può contemporaneamente essere contrastato dallavanzamento della linta di spiatgria.

Si tuatia comunque di equilibri instabili, talvolta labili, cle lievi alterazioni delle forze in grinoco possono infrangere: p. es., la modifica del regime di alcuni fiumi, provocata da sbarramenti artificiali, du, senza alterare la lentac diminuzione - per insabbiamento - tella profondità media dlel bacino (nel caso specifico, l'slto Adriatico), non consentit un efficace contirnsto per apanzarnento (li spiaggia ("*).

consitlerato finito: resta quindi solamente I'oscillazione forzata propriamente Ietla.

Ju secondo luogo, l'atdito produce una lieve diminuzione d'ampiezat c un piceoln sfasamento (11). Inolire, osserveremo una risonanza meno perfettal, Infatti, ahhiamo

$$
\varepsilon_{r}=\Sigma \frac{D_{j}}{\lambda_{-}-\lambda_{j}},
$$

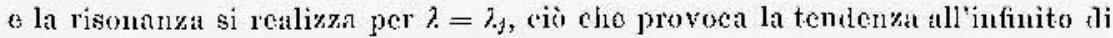
uno dei termini, che prevale pertanto decisamente su tut ti gli altri. Con lattrito, la risonanza non sarà più ensi perfetia. Infatti, mentre $\lambda$ è puramente immaginario, essendo le forze perturhatriei essenzialume periorliehe, $\lambda_{f}$ invece la una parte reale nestativa. Pertanto $\lambda$.... $\lambda_{\text {s. }}$ non si annulla mai.

In pratica, però, la differenza non ì granide, perehé la parte reale di if ¿ sempre estremanente piecola in valore assolutio; ne eonsegne ehe $\lambda . \lambda$, potrà diventare piccolissima, il che consentirà una risonanzi quasi perfetta.

(*) L'ampiezza delle oscillazioni nellalto Aslriatieo può subire - a pariti di altre eoudizioni - un incremento anche per unaltra causa: il progressivo restringimento del bacino $\left({ }^{26}\right)$; cic che - non ostante il braldisismo in atto -- effettivamente (anclee se lentissimanente) si verifica, con liavanzamento, sopra un fronte di parecelie diecine di chilonetri, del belta che si inoltora senpre più in mare [nclla fase a delta lubato, di cirea $70 \mathrm{~m}_{i}$ anno (it)]. 


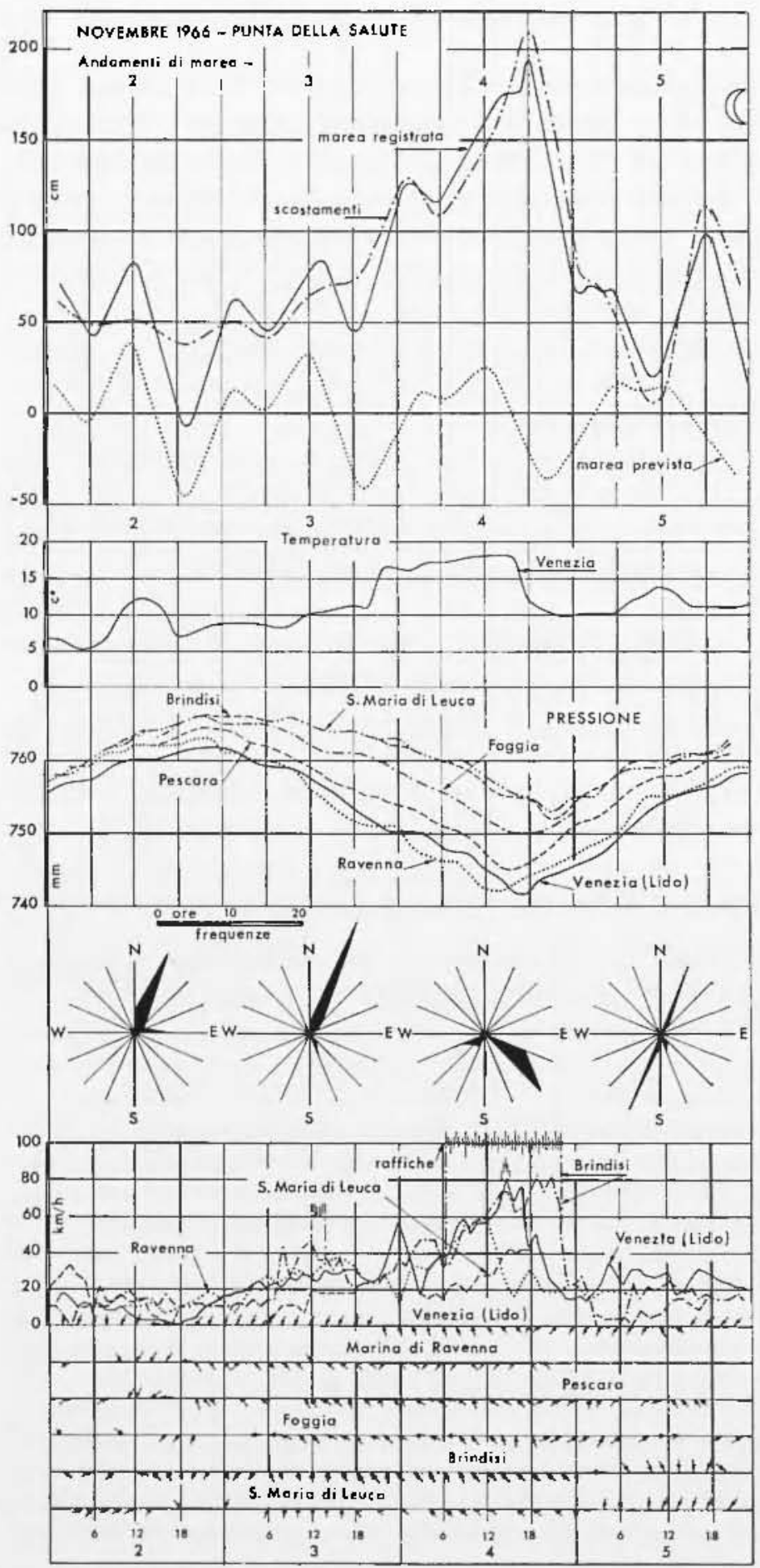

Fig. 23 -

Acriua alta

a Venezia ai mimi di No-

rembre

1966. Jati mareografici e meteorolo. gici [Secondo L. Torigo $\left.{ }^{(58)}\right)$ ].

Fig. 23 -

Higlı water in Venice early in No. vermber 1966. Tide gauge and meteorolorrical data [After $\mathrm{L}$. Dorigo ( $\left.\left.{ }^{58}\right)\right]$. 
c) Come si è più volte accennato, in seguito alle disordinate estrazioni di acque metanifere, praticate nel belta palumo th partire dal 1948 - estrizzioni rontinuate fino al $1960 \mathrm{e}$ tuttora in atto in zone uppena esteme al Delta - una vasta zoní del Polesine - già naturalmente in lento abbassamento - las subito notevoli flessioni, che in talune localitì hanno superato i 3,5 metri.

Questi spuofondamenti labuno necessariamente interessato anche la zona costiera, e il fondo del mare in prossimità di essa (*). Analogo fenomeno - legato a cause conconitanti, c, in parte, alla stessa causa si è verificato in corrispondenza delle coste dell'alto Alriatico, da Vonezia a Ravenna (**) .

Da. 195., Jungo la costa occilentale del golfo di Venezia, è quindi in eorso una progressiva, lieve diminuzione dell'angolo fra il fondo marino e la superficie dell'acqua, nei pressi della riva. Tella dinamiea dei moti lenti del mare (leguti al transito ai variazioni di pressione) ciò si traduce in un aumento relativo dell'ampieza del movimento, in conformita tella teoria esposta al n. 6, la quale prevete che fenomeni ti risonanza possono manifestarsi nella propigazione di un moto ondoso verso da costa, quanko il fondo marino tende and essa con dolce declivio (***); e che tali fenomeni sono tanto più vistosi quanto più il fonclo matino tende a disporsi parallelamente alla superficie dell'acqua.

(*) Ciò è provato dlalle isocinctiche (dla -20 e -70 em/anmo) aperte snl mare, non solo chunts il periodo dei copiosi emungimenti, ma anche dopo l'interruzione degli stessi nella zona tlel Tolta (isocineticlic da -10 a -50 em/anno), e ancora in atto nel 1967 [figg. $2-5$ di $\left.\left(r^{72}\right)\right]$.

(**) A Porto Corsini, tlal 1950, è in ammento la frequenza annuale di acque alte, senza dubbio anehe a motivo dell ablossamento slel suolo, isí í atto. A riprova, si osserva che le alte marec maggiori o uguali a $\mathrm{m} 1,00$, seguendo ma eurva di sempre magriori frequenze, al prineipio più lenta di quella di Venezia, a partire dal 1962 si fa di anno in amno più ripisa, superando largamente il numero annuo di aeque alte osservatio a Venezia. Cosi, mentre dal 1950 al 1961 (in 12 anui) il numero delle acepue alte fu a Venezia di 52 unità e a Porto Corsini di 26 , chal 1962 al 1969 (in otto anni) a Venezia si ebbero 66 a.a., mentie a Porto Corsini il loro numero sali a ben 144 [p. 54 di (79)]. Nel volgere di pochi ami, quindi, si è capovolto il rapporto delle frequenze nelle the localiti.

(***) Naturalmente, il Aeclivio può variare da zona a zona. l’er quatuto ríguarla il Delta, p. es., si osserva un più rapira ammentio della profoudità, challa costa verso il larro, " nella fascia sot to costa compresa fra l'orto Calerie Punta della Maestra. Piì precisamente, mentre le isobate fino ai $15 \mathrm{~m}$ si mantengono più o meno alla stessa distanza clalla costa, le isobate dai 15 ai 
I] caso sturliato flai giajponesi (32), e riassunto nella fig. 12, sotto molti aspetti sembra scelto su misura per l'alto Aclriatico: le profonclità in siuoco sono infatti le stesse. Anche per lalto Arliatico, le velocitì di transito rlelle perturbazioni meteorologiche, alle quali corrispontono fenomeni di risonanza, sono dell'orline di $/ g h_{0}$ (per $h_{0}$ variaute fra 20 e $25 \mathrm{~m}$ ) (2). Sotto due aspetti la situazione dell'alto Adriatieo is perc nettamente peggiore. $\left.1^{\circ}\right) \mathrm{ll}$ caso esaminato dai giapponesi presuppone, fra le profontlita estreme di $10 \mathrm{ml}$ e di $8 \mathrm{~m}$, una distanza (in proiezione) fi soli $20 \mathrm{~km}$, mentre, per l'alto Adriatico, tale distanza varia fra $40 \mathrm{e}$ $90 \mathrm{~km}\left({ }^{75}\right)\left(^{*}\right)$; quindi, con una pentenza media del fondo nettamente minore di quella considerata flai gianponesi, il che comporta - a parità di altre condizioni - ma ben pili accentuata ampiezza dell'intumescenza liquita che procede verso la costa. 20) Con l'aggravante, in conseguenza del sensibile abbassamento della costa occidentale in atto dal 1950, di una progressiva intensificazione - sempre a parita di altro condizioni - dell'effetto di risonanza.

Consirleriamo, ar esempio, l'acqua alta verificatasi a Venezia fra il 3 e il 5 Sovembre 1966. Ialla fig. 23 risulta che, in quei giorui, la variazione di pressione fu di $8 \mathrm{~mm}$ di mercurio ca. Ar essa corrispose come da fig. 24 - un innalzamento del livello thel mare di $90 \mathrm{~cm}$ ca, cioc quasi nove volte il valore statico!

d) Come si è rletto al $11 . \bar{\imath}$, dal 1940 ì in atto un'inversione di tenrenza rlella temperatura dell'aria sulla Terra. $\Lambda$ d essa ha fatto riscontro un'acentuaione dei fenomeni meteorologici, con particolare riguarto alla pioggia.

25-30 m subiscono un progregsivo allontanaumente procedendo da nori verso sud. Infatti l'isobata dei 25 m a nord di Punta della MIastra, dista dalla costa in media $6 \mathrm{~km}$, a sud arriva ar un massimo di $11 \mathrm{~km}$ all'altezza di porto Folano" [Cialuatti $\left({ }^{75}\right)$, p. 191]. Fiò dipende talle caratteristiehe degli apporti a mare o dal ginoco delle correnti. Cosi, a nord di punta della Maestra i sedi. menti hanno una granulouetria meno fine che a sud, mentre l'unpia zona sahhiosa "si sviluppa verso nord oom matura diversa e più alta granulometria. Da tale fatto si può supporre rhe i corsi d'acequa a nord tel Po, portino al mare materiali jiù grossolani e di matura diversa, anche se è visilile un trasporto verso sud dei materiali piic fini ad opera delle correnti suheostieren $\left[\left(0^{74}\right)\right.$, p. 208$]$.

(*) Sopra un fronte di cirea $100 \mathrm{~km}$, l'isohata di $50 \mathrm{~m}$ dista da quella tli $40 \mathrm{~m}$ fra i 25 e i $50 \mathrm{~km}$; la zona fra le due isobate può quindi considerarsi un'estesa pianu'a orizzontale. 


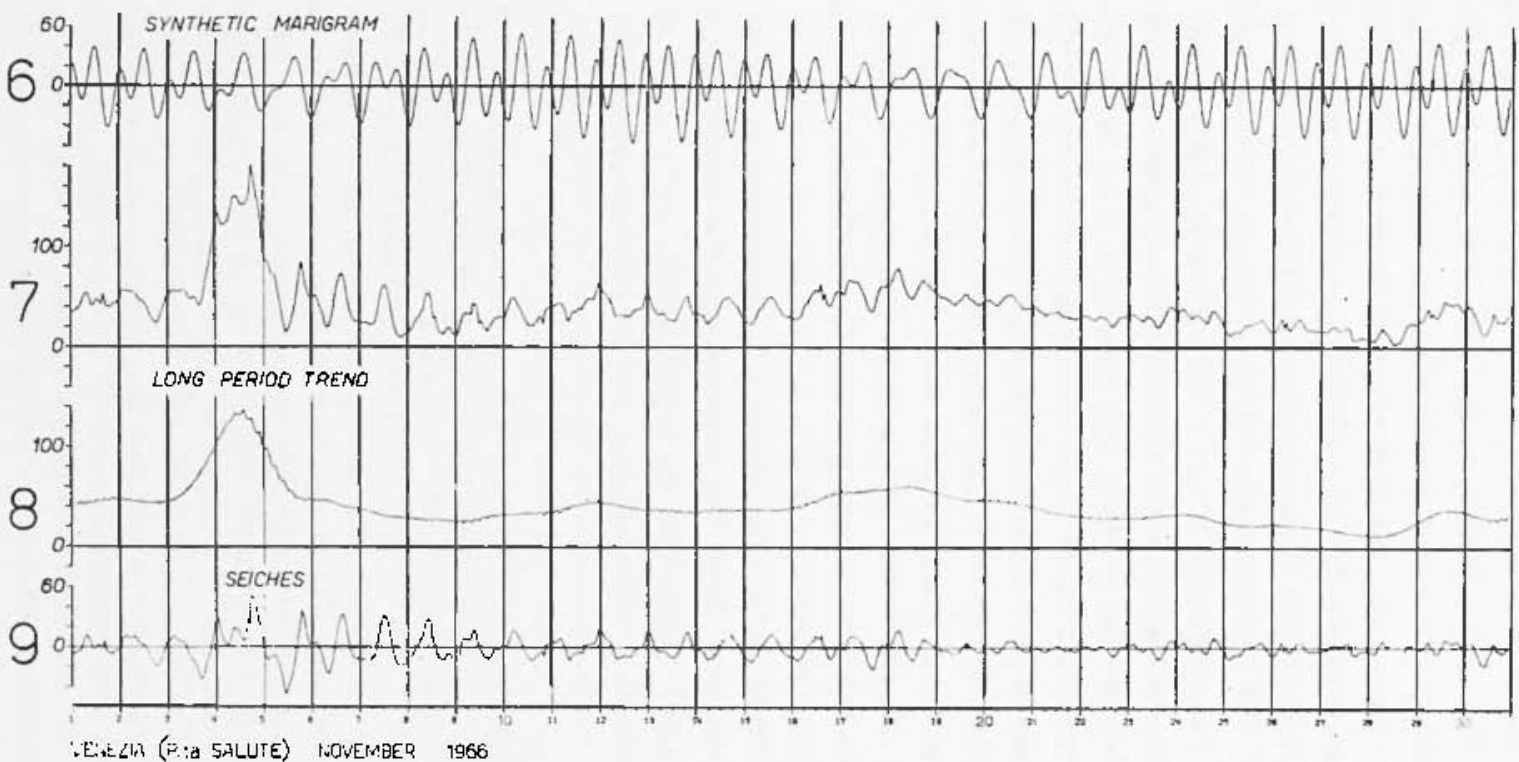

Fig. 24 - Parte del risultato kellapplicazione dellinalisi periolale (metoslo Mosetti), uslesa a tutto Fovembre 1966. Si ripoltano solo i tratti linitati ai grafici da 6 a $9:$ 6) mareogramma previsto; 7 ) mareogramma resiluo (eomposto da 8 pit 9); 8) componenti a lungo periaklo; 9) residue oscillazioni libere e forzate (secondo Mlosetid-Carrozzo $\left({ }^{76}\right)$ ).

Fig. 24 - Part of the results from a period analysis (Mlosetti method) extended over the whole month of Novenber 1966. Only the tracts referring to graphs o t.o 9 are sliowu: 6) marigram she to the astronomical [orees; 7) resiklual narigram (made mp by 8 plus 9); 8) lontr periol eomponents; 9) free and forcel residual oscillations [after Iosetti-Carrozzo ( $\left.\left.{ }^{78}\right)\right]$. 
Sebbene non si trovi cenno di tale grandioso fenomeno nei lavori compiuti sul problema di Venezia, sta di fatto che il Magistrato alle Acque ha riscontrato, negrli ultimi decenni, un sempre più frequente ripetersi di precipitazioni atmosferiche nella zona associata all'alto Arlriatico (n. 9.2); inoltre, si ì andata intensificando la frequenza dei venti e la loro durata, specie per quanto si riferisce ai venti di SF-SSE (sciroceo), come is provato dalla fig. 24, pubblicata dal Prof. Gioriani Soika, direttore dell'Osservatorio meteorologico dell'Ospedale al Mare (Jido), e trattu da (18). Più recentemente [1970, v. $\left({ }^{(3)}\right)$, p. 55] Giordani Soika e D. Meneghini hanno precisato che a tali variazioni, più accentuate e più significative nell'ultimo quinquennio, si concretizzano in un forte aumento della bora nel periodo antunnale (...) ed in un aumento del vento di sciroceo in tutto il periodo estivo-antunmale ". Gli AA. ritengono die cio abbia provocato un aumento nell'insorgenza delle sesse. $A$ questo proposito - come diremo ampiamente più sotto - ̀̀ da ritenere clie l'intensificarsi del fenomeno delle oscillazioni libere (o forzate) non sia tanto da attribuire alla maggior frequenza dei venti, quanto all'aceentuarsi della loro causa prima, che si identifica nelle variazioni di pressione atmosferica.

In ogni modo, l'acentuarone dei fenomeni meteorologici, legnte all'inversione dellid tendenza della temperatura media dell'aria nel mondo a partire dal 1940, comporta un'accentuarione delle cause di perturbazione del livello del mare, legate al eventi atmosferici (*).

(*) A rigror di termini, per ottencre un livello metio tel mare di sicuro riferimento, occorrereb be eorreggerlo dalle alterazioni associate alla pressione atmosferica, al vento, all'evaporazione, alle variazioni di temperatura, ... Iavoro non facile, invero. Certo, le piu cospicue alterazioni sono quelle legate alla pressione, anche perehé il vento ne è ma conseguen\%a. Per quanto encerne le variazioni associate alla pressione, per eliminate o riclure al minimo le eorrezioni, si suole ricorrere ai valori mexli đlecennali. Ciò vale però per le variazioni statiche. In easo di interazioni aria-acqua nel eampo della risonan\%, tali correzioni non sono più valikle: in ogni easo, saranno sempre insufficienti. Il fatto che in clue decnni suecessivi la pressione atmosferica abbia medic varianti solo di deeimi tli millilsar, non antorizza a concluderne clie il l.m.m. non abbia sulsito variazioni sensibili da parte tella pressione atmosferica: l'esistenza di eventuali varia\%ioni dinamiehe, mascherate $\mathrm{c}$ mediate, portano at alterazioni del $l . m . m$, the in realta non dovrelbbero figurare. Lin'alterazione clinamica, del tipo selezionato in fig. 24, avente la forma di un'clongazione - d'altronde mascherata in una l'egistrazione complessa, come da fig. 23 - - nella pratiea eorrente non viene che parzialmente corretta. Sie joi queste alterazioni erescono di frequenza nel tempo -..- come 
Di qui l'interesse rlegli sturli relativi all'internzione fra atrosfer'u erl idlosforit.

Un'opinione, ancor'n molto diflusn, fa ritenere il vento come cuusa fondamentale della formazione delle oscillazioni liber'e ali bacini liquidi, chiusi od aperti. Si suol dine the tali oscillazioni insorgano quando il vento, dopo aver soffiato a lungo, e nolla stessa direzione, verso una costa, cala bruscamente d'intensità. Se tale causa di futto esiste, essa è del tutto accidentale $e$, in ogni caso, di elfetti secondari. Nei lunghi anni cle lio dedicato allo studio e all'osservazione di questo fenomeno, ho avuto modo di provare che la causa principe (se non unici) delle oscillazioni libere (sesse) va ricercata nei gratienti della pressione atrosferien. Sovente, il transito delle variazioni rlella pressione atmosferica sopra un bacino is accompannato da venti, più o meno forti; forse, $i$ tale coincidenga che fa attribuire a questi ultimi gran prite rell'effictcia, atta a sviluppre oscillazioni libere o forzate; cffeacia che spettid invece, in modo prodominante, alle perturbazioni di pressione, tanto è vero dus talora il vento presenta azione smorzante su oscillazioni in atto (30).

Vuriazioni di pressione, anche minima, transitando su bacini i più diversi - per forma, rlimensione e profondita - vi eccitano oscillazioni coperiodali, proprie dei bacini stessi $\left({ }^{5}, 78\right)$, od oscillazioni forzate $\left.{ }^{77}\right)$, anche in mancanza di vento (5) (o con vento debolissimo $v$, fige. 1, 2, 3, 6). Cio ho avuto modo di constatare sia in piceoli bueini chiusi, sia in mari estesi. Quando il yitmo della variazione della pressione i: molto prossimo a quello delle oscillazioni libere delle aeque sotitostanti, bastano gradienti barici irrilevanti, dell'ordine di frazioni di Tow (e quindi registrabili solo con sensibili microbarografi) a provocare — in assenza di vento - ampli movimenti coperiodali nei bacini (5). E tali movimenti possono verificarsi sui ritmi di una gamma estesissima, da quelli con periodi di pochi decimi di secondo che, verificandosi alla superfieie dei due mezzi interagenti possono dar luogo ai rricrosismi $\left({ }^{7}\right)$, a quelli lentissimi, capaci di mettere in moto le acque interne di un grande lago [come il Garda, che durante i mesi estivo-autumali, formatasi la. superficio rlel salto termico, oscilla per settimane c settimane con periodo

a Venezia, e a Porto Corsini - il livello medio del mare (valutalo con i metoti tradizionali) non polrá che essere un livello molio fittioio, destinato at alterarsi vieppiù nel tempo anche in forza di efteti isostatici. is quindi piu grinsto tesinire il l.m.m. di questi ultimi decenni, come livello nedio apparente. 
di 24 ore chro - quello della sua binodale interna, eccitafa dalla coperioulale oscillazione barometriea diurna $\left.\left(^{4}\right)\right]$.

IIa tomiamo all' $\Lambda$ driatico.

In un recente lavoro, $\mathrm{F}$. Mosetti - uno dei più attivi e fecondi esperti del cosi tletto "Comitatone " ministeriale -, Accerboni e Castelli

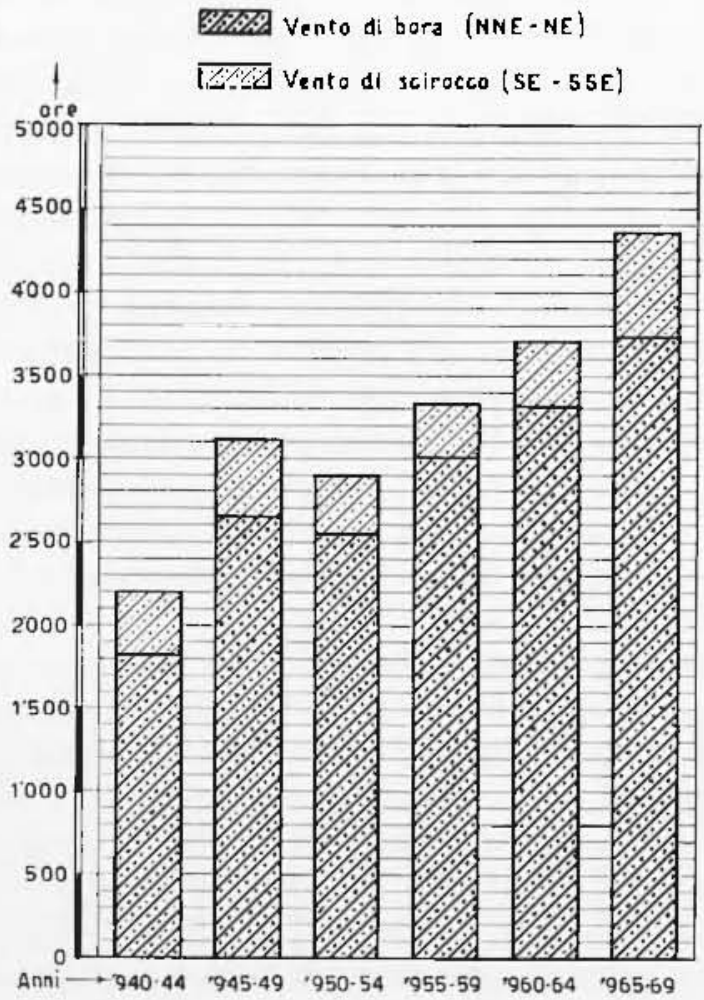

Fig. 25 - Ore totali di rento (born e sciroceo) lal 1940 al 1969 , registrate a Venezia-Jidn (Becondo Ginrlani Soika).

Fig. 25 - Total of wind hours (hora plus sciroceo) from 1940 to 1969 , recorder at Venice Lislo (after Giortani Soika).

hanno dedieato nuo stadio all'azione del vento sull'elevazione del livello nell'Arriatico settentrionale, estendendo a questo mare molelli già utilizzati da altri autori ad altri bacini $\left(^{80}\right)$. Gli AA. ritengono che "la soluzione del problema mette in evidenza il sollevamento di livello ehe si produce jer clietto del vento e la conseguente apparizione delle sesse con perioto di circa 21 ore, che sono in perfetto accordo con l'osserva- 
zione " (1.e., 1) 18). L'applieazione fatta alla mareggiata del 3-1-5 Novembre 1966, con riferimento a Venezia, è riassunta nellà fig. 26. Nella didascalia è detto che "se si trascura l'eftetto delle sesse a periodo pressuppoco scmidiurno, delle quali non istato tenuto conto, si nota la notevole corrispondenza dei due diagrammi " (1. e., p. 29). Tale corri-

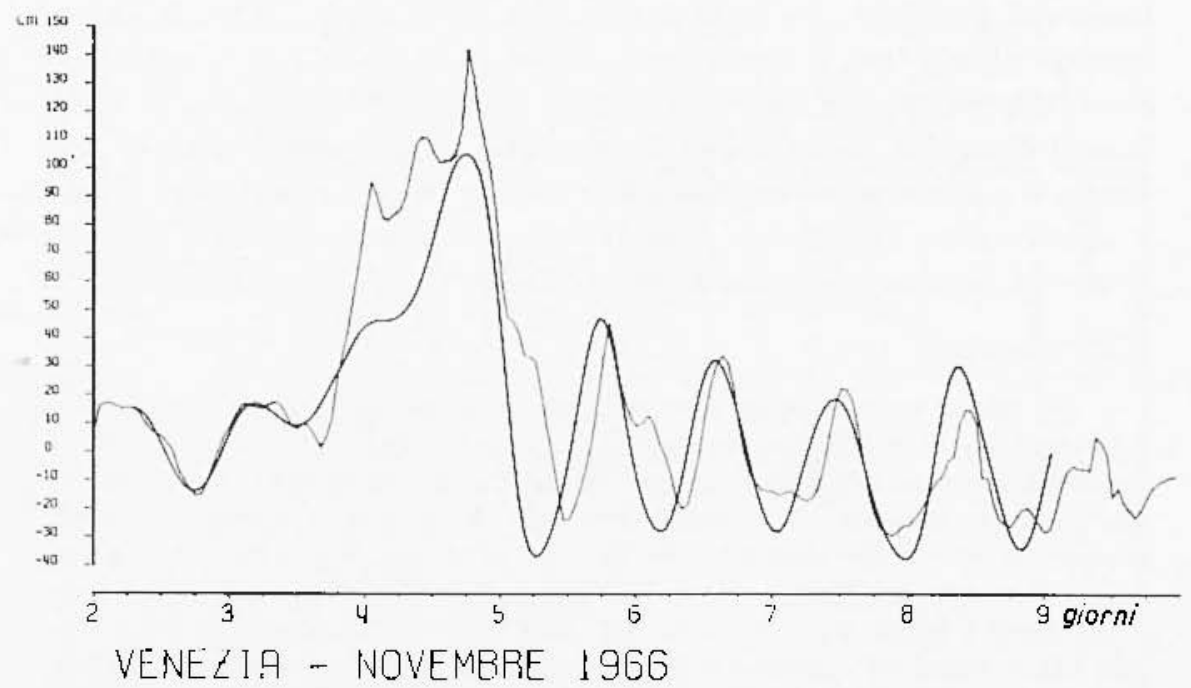

Figr. 20 - Componenti dell'aequa alta (linea mareata) dei primi di Novembre 1960 a Venezia, quale sarehbe stata causata dal vento di seirocen [secomdo Accerhoni el al. (80)].

[Nella fig. 24 i riportato quale fu in realti - nelle suc componenti a lungo e a hreve periodo - lo spostamento anomalo complessivo, dovuto ad agenti meteorologici; in particolare, alle variazioni della pressione atmosforica].

Fig. 26 - Components of high water (marked line) of the first Fovember days of 1966 in Veniee, saif to he cansed hy the sciroeco wind [after Aceerboni and others $\left.\left({ }^{(8)}\right)\right]$.

(Fig. 24 shows, in its long and short period components, the total anomalous displacement, what in reality was, due to weather agents, particularly to variations of atmospheric pressure).

spondenza è però solo ajparente. E lo prova lo stesso Rosedti in un altro lavoro (condotio con la collaborazione di M. T. Carrozzo), apparso sul medesimo numero del Bollettino di Geofisicu ( $\left.{ }^{76}\right)$. Tn questo lavoro viene presentato un nuovo, veramente efficace metodo a analisi delle maree. L'applicazione di questo metodo alle mareggiale dei primi di Novembre 1966 ha condotto alla separazione delle varie componenti (fig. 24). Dalla figura appare chino che l'acqua alta a Tenezia è in gran 
prerte associata all'intumescenza, whe si sviluppa rlurante tre giorni cirea, provosatu dalla contemporanea fessiome della pressione atmosferica ca esaltata per fenomeni di risomenza, per il verificassi rlelle conrlizioni di (ui al n. 6. Fssa si presenta come un'elongazione, conformemente alla ricorrata teoria (fig. 12). ('ome risulta dalla fig. 24, sovrapposta all'intumescenza principale, vi è tutta una serie ri sesse; fra le quali presiominano nettamente le semirliume, ahe si presentano con la caratteristica di ampi impulsi isolati (propri bei bacini aperti), mentre le oscillazioni di 22" a - anch'esse thiscontinue — appaiono di piccola ampiezan e comunque soverchiate ralle semiliurne. In ogni mous, le une e le altre sono ginstificate dalla presenza rli coperioulali variazioni di pressione, resa manifesta dialla curva barometrica di Tenezia (fig. 23) (*).

(*) flic il vento non sia iletcrminante nella formazione ilelle acque alte, is provato anclie dalle aller mategriate, messe a confonte con le condizioni metororoloriche: in eni si sono verificate. I. Dorigo ( $\left.{ }^{81}\right)$ mette a confronto 11 alte matee verificatesi a Venezia (Punta della Salute), con gli clementi meteorologici a cui furono associate. Il 16. IV. 1936, a.a. em 147, pressione al colmo di marea mm 742,5 , vento $54 \mathrm{~km} /$ ora da $\mathrm{bSW}$, cioe con forte componente verso il laren (fir. 27a); il 12. 11 I. 1937, a.a. om 119,5, pressione al colmo mim 752,0 , vento 66 km/ora dal $3^{\circ}$ quadrante, cine trasversalmente all'Ailriatico; il 5. XII. 1946, a.a. cou 104, preskiome al colmo mm 746,8, vento 24 km/ora da $\mathrm{ENE}$; il 9. X11. 1946, a.a. en 136, pressime al colmo 1 m 741,8 , venti uleboli dal 30 e tal 10 quadiante; il 29. XI. 1947, a.a. ('m 126,5, pressinne al colmo mm 747,8, vento debolissimo (8 km/ora) da SSW; il 27. 1. 1948, a.a. em 119,5, pressiont al colmo mm 750, venti moderati orientali; il 28. I. 1948,

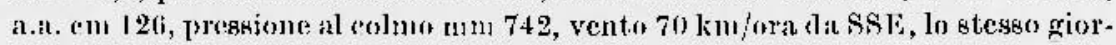
no (101" sopo), a.a. ("m 132, pressione al colmo mm 745,5, vento $20 \mathrm{~km} / \mathrm{ora}$ tranversale all Ariation (da WSW); il 12.XI.1951, a.a. en 151 (fig. 27b), pressione al colmo mm 742 , vento 48 kmiorat ta SSW; il 24.XIJ.1958, a.n.

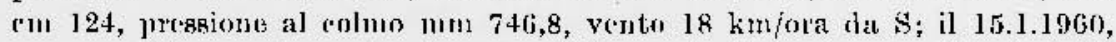
a.a. cm 126, pressione al colmo nm 750 , vento $34 \mathrm{~km} / \mathrm{ora}$ da $\mathrm{NF}$ (quindi, tendente a "deprimere $n$ il l.m.m.); il 15.X.1960), a.a. cm 145 , pressione al

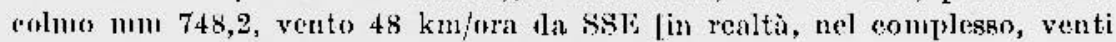
predominanti kel $3^{\circ}$ qualrante, da moderati a quasi forti (fig. 27c)]; il 5 Novenbre 1960, a.a. chi 123, pregsione al colmo mm 745,5 , con vento ali briti $8 \mathrm{~km} / \mathrm{ora}$ da $\mathrm{N}$ (fig. $27 \mathrm{l}$ ).

L'clemento in crmunc di tutte quente alte maree è esclusivamente la bassa pressione. Il vento, generalmente delsole, può provenire dialle più diverse lirezioni. L'acyua è lanto piì "alla "quanio più "fonda "è la flessione della pressione ntmosfericn; e quindi quanto maggiore è il sus gradiente prima e doqo il minimo, o quanto piu prossimo è il sto andamento alle condizioni di risonanza. Apprunto come si ì verificato fra il $3 \mathrm{e}$ il 5 Novembre 1966 , che - sotto questo is]etto - può essere comsiclerato un caso limite. 


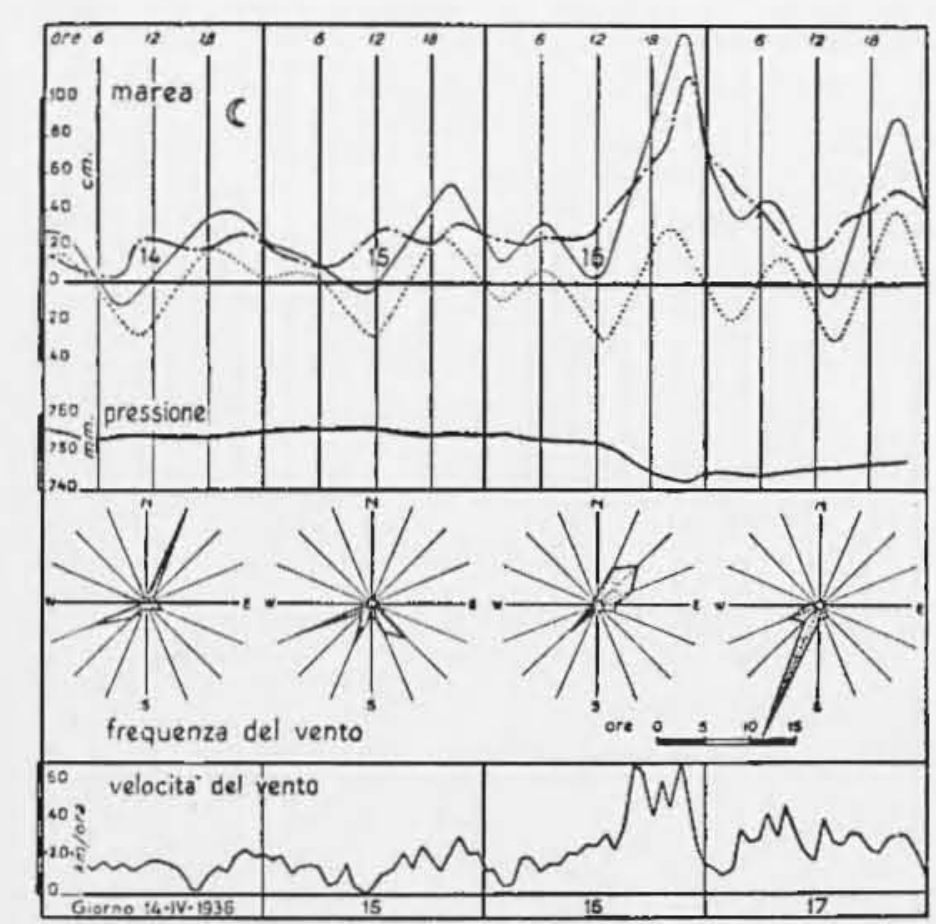

a)
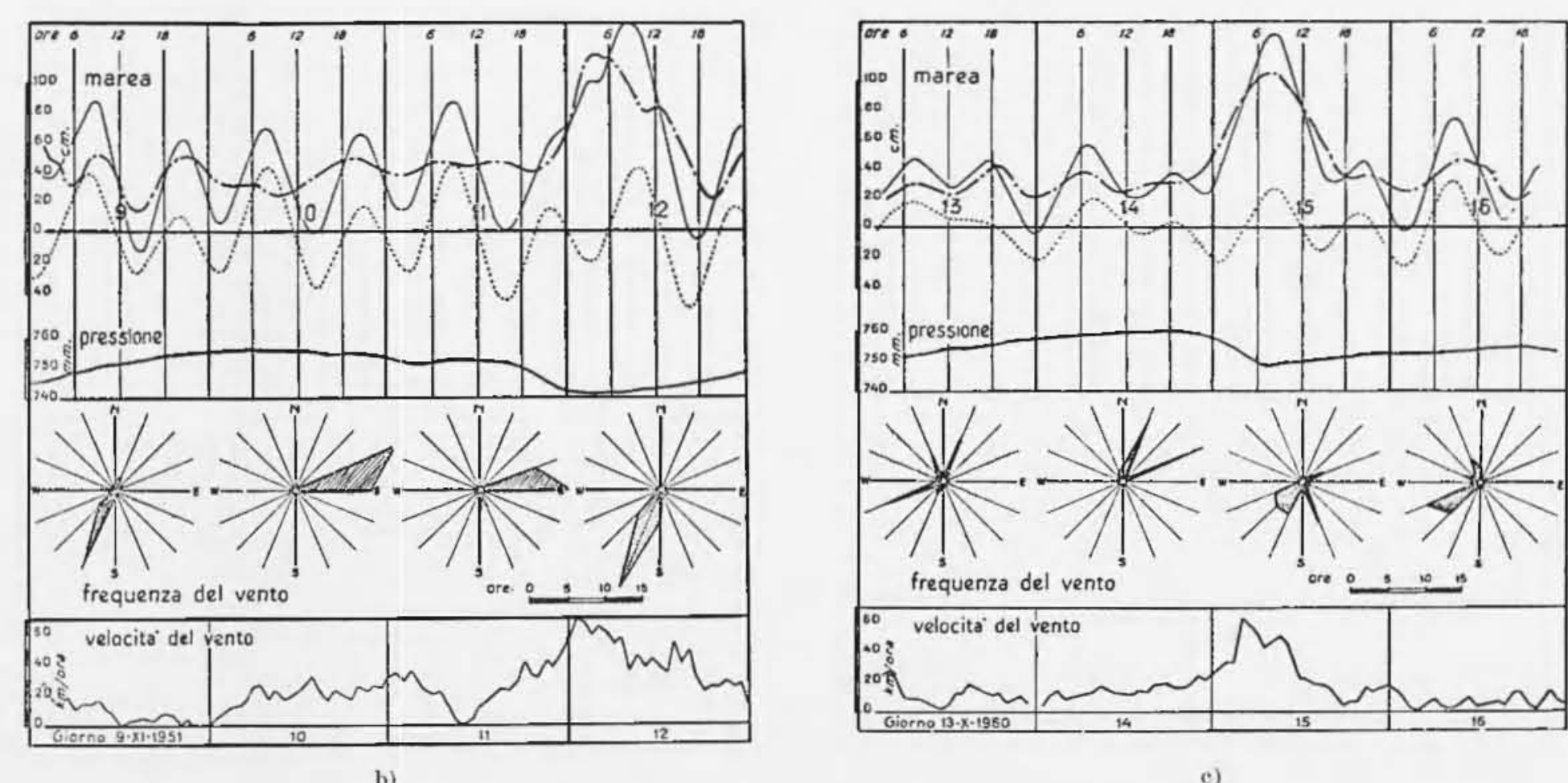

c)

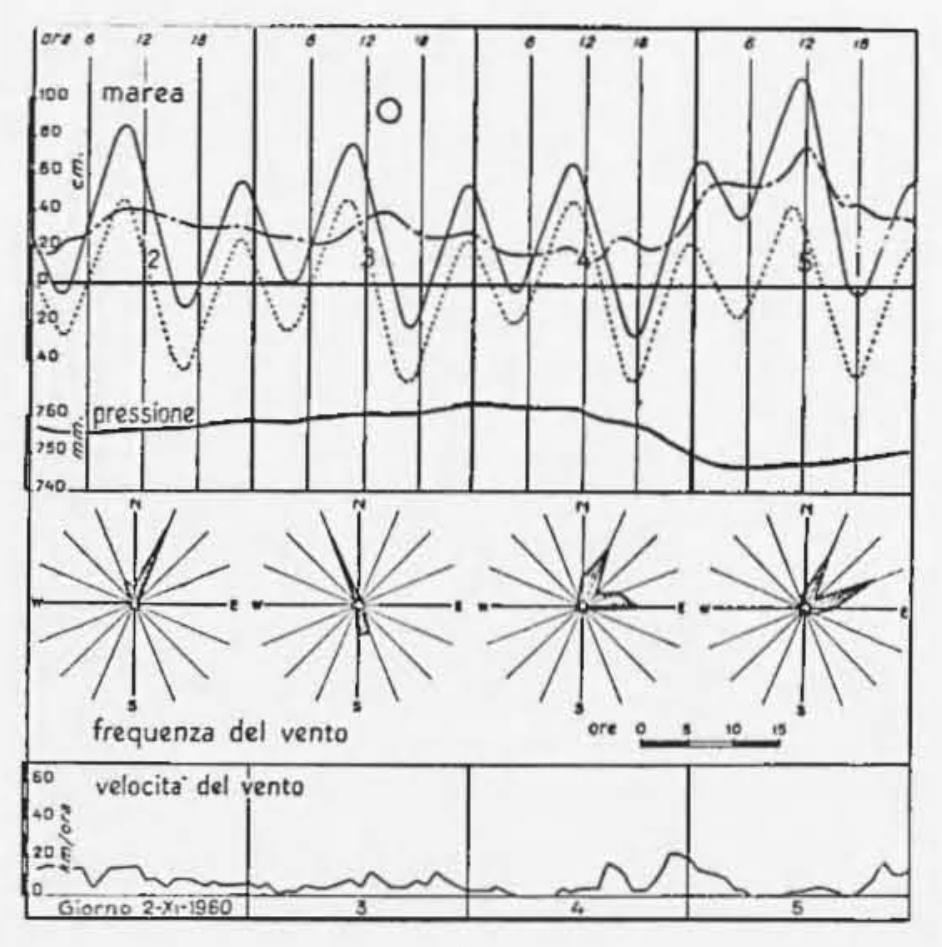

d)

Fig. 27 - I diagrammi a) b) c) d) ed altri che si ritiene superfluo riprondurre [tratti da un lavoro di L. Dorigo (31)], provano che la causa predominante (se nou esclusiva) delle alte marce eccezionali è sempre quella legata alla bassa pressione atmosferica sull'Alto Adria-
tico: esse possono infatti rerifcarsio o in maneanza di vento, o con vento dai quadranti settentrionali o da quelli oceidentali.

Fig. 27 - Diagrams a) b) c) d) and others here unreported, taken from a study by L. Dorigo (a1), prove that the prediomimant, if not exelusive cause of the exceptional high waters, is always connected with the low atmospherie pressure on the High Adriatic. They are apt to originate either without any wind or with winds from the Northern or the 
Le variazioni rli pressione sono quinti suffeienti a spiegare il fonomeno dell'sequa alta a Venezia, almeno nei suoi aspetti predominanti. Del resto, dalla fig. 23 risulta chiaro che il vento ha avuto balzi di intensitì e cli direzione in parechie delle stazioni d'osservazione lungo la costa occidentale adriatica, il che comporta multiple soluzioni di continuiti nella sua azione. Che le sesse non siano legate al vento è provato dal fat to che esse sono presenti, non al cadere del wonto [come i rletto a 1.32 di $\left.\left(^{8}\right)\right\rceil$, ma prima cle esso inizi a soffiare da SE e nel pieno della sua azione locale; e che raggiungono lo massime ampiezze in corrispondenza di coperiodali sovrapposte variazioni di pressione e della brusca. inversione di tendenza di quest'ultima, per fenomeni analoghi a quelli rappresentati dalle figg. 1-6.

Sta di fatto che, da. 1951, la frequenza delle "acque alte "nella laguna di Venezia ha subito un forte incremento. Tse brusche alterazioni del fondo, legate alla grande alluvione dello stesso anno e a quelle clegli anni successivi - protese ad elevare l'ampiezza dei movimenti marini e ad allungare il periodo delle oscillazioni libere dell'alto Adriatico la lievitazione delle acque susseguente (per diminuita salinità) ai periodi di piena ed agevolante l'intensificazione dei moti dell'acqua in campo dinamico, unite all'azione di rinforzo consegnente all'abbassamento delle coste occidentali, le une e l'altua potenziate dall'accentuarsi delle perturbazioni meteorologiche, possono a ragione essere invocate fra le principali cause dell'allarmante fenomeno.

Di fronte ad eventi tanto grandiosi, c'è da domandarsi fino a che punto limitati lavori eseguili nella Laguna possano influire sull'entilit delle mareggiate, quando queste si presentano con l'ampiezza clie si riscontra in mare apertio. Il dramma di venezia, va pertanto visto sullo sfondo di fenomeni naturali di non facile controllo da parte dell'nomo; ignorare questo aspetto del problema, significherebbe rinunciare in partenza ad una sia pur larvata sua soluzione.

\section{BIBIJOGRAFIA}

(1) Calo P., 1963. - Interazioni tra atmosfera ed idrosfera. "Ann, di Geofisica $"$ XVI, pp. 1-35.

(2) Calor P., 1938. - Sesse dellalto Aariatico, con particolare riguardo al yolfo di Trieste. R. Comit. Talass. Ital, Memoria CCXI,VII.

(3) Calor P., 1961. - Sulle onde interne del lago di Bracoiano e sulle toro probabili cause, "Rend. Ace. Naz. dei Iincei, Classe Se. fis., mat. e nat." Seric VIII, $\mathbf{X X X}, 5$. 
(4) Cator P., 1963. - Te onde interne del lago di Garda come effetto di interdzione fra atmosfera ed idrosfern. "Renkl. Aec. Naz. Ilei Jincei, Classe Sc. fis., mat. e nat, ", Serie VIII, XXXIV, 2.

(5) CaLor P., 1972. - Uleriori esperichze geofisiche sul lago di Caldonazzo. Morjologin ed origine delle sesse . . " Ann. tli Geofisiea "XXV, 2.

(0) Ravirici C. - Le lagune renete sono un resto dellantiea Laguma adriuna: ragione della loro sussistenza. Vuntzia (s.ll.).

(7) Doktio L., I965. - Amali Ldrologici 1964. Parte seconda. Istituto Poligrafico tello Stato, P. 125.

(8) *** 1878. - Caria Generale del Mare Adriatico. Regria Marina Italiana e I. R. Marina Austro-Ungarica 1867-1873. Foglio I, Gienova, Ist. Islrogr. della Marina.

(9) *** 1904. - Adriatioo da Aneona a Fiune 1896 (1928). Genova, Ist. Idrogr. R. Marina.

(10) Moswert F., 1968. - Morfologin dell -1driatico settentrionale C.N.R., "Coum. Sturlio per Ocean. e Timm, serie B, N. 16.

(11) Pornchú H., 1910. - hesons de Mécanique Celeste, Tome IY. Theorie des Marées. Paris.

(12) Toaldo G., 1781. - Della vera infuenza degli Astri sulle stagioni e muazion di tempo: Sagyio meleorologico, PI. 230. Stamperia del Seminario, Padova.

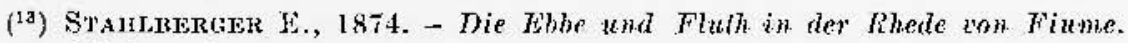
Butapest.

(14) Grablowitz G., 1892. - Le maree nell'Alvialico. "Neptunia ", Venezia.

(15) vos Sterneck R., 1904. - Die Hohe des Milleluctsers bei Ragusa und die Ebbe und Flub in Adriatischen Meere. "Mitteilungen des K. u. K. mil, geograpl. Inst." XXIII, 1903. Vienna.

(10) voN STHiseck R., 1908. - Das Fortschreiten der Fluthwelle im Adrialischen Meere. K. Akarl, d. Wissensch., Vienna.

(19) voN STE1NECK R., 1915. - Zur hydrodynamischen Theorie der Adrajezeilen, "Sitzb. \{. K. Akakl, «. Wissenselt. in Wien, Math.-naturw. ", Bil. 124, Yienna.

(19) vos Kesslitz $1 \%$, 1910. - Das Gezeitenphänomen im IIafen von Pola. "Mitteil. ans dem Gebiete des Seenwesens ", B\&l 38, Pola.

$\left.{ }^{10}\right)$ vox Kessuta W., 1912. - Ilas Gezeilenphänonen in Hafen von Ragusa. "Mitteil. aus dem Gebiete des Seewesens", Bil. 40, I'ola.

(20) Defaxt A., 1911, - Uber die Periodendaner der Eigenschwingungen des Adriatisehen. Heeres. "Annalen der IIykrographic und Maritimen Meteorologie ", X[ïrz.

(21) Obbose E., 1910. - Alowne ossernami idrodinaniche su di wh piccolo modello di mare Adriatieo. "Annali Liff. Centr. Meteor. e Geodin. Ital. ", XXXII, Parte I.

(22) Vercend. F., 1922. - Sulla previsione det termini di eorrezione nelle maree. "Rivista Marittina ", Febbraio. 
(23) Por.u. S., 1958. - Te sesse (seiches) dell'Ariatico. "Ann. di Geofisiea " $\mathrm{XI}$, p1. $69-76$.

(24) BaJc C., 1972. - Seiches in the driatic Sca; a theorefical approach. "Boll. Geof. tcor, e appl, n, XIV, X. 53-54, p1. 25-33.

${ }^{25}$ Kasumove M., 1959. - Der Dymamische Einfluss der Almosplä̈re auf die Seespiegelschwankungen der Adria. "Südslaviselte Akadenie der Wissenschaften und Kunst, Alshandlungen n, 33\}. IJ, X. 1, Zagrely.

$\left({ }^{26}\right)$ Caror P., 1951. - Oscillazioni libere del layo di hevico. "Ann. di Geofisica ", IV, pp. 193-206.

(27) CaLor P., 1954. - Oscillazioni libere del Lago di Garda. "Arehiv für Meteorologie, Geophysik und Biolilimatologie ", Serie $A$ : Meteor. u. Geo. pliysik, Band $\bar{t}$, p. $+6 \mathrm{l}$.

(28) Cator P'., Spadea M. C., 1961. - Sulle oscillazioni libere del Mar Ligure. "Ann. di Geofisica" XIV, 2.

(29) Calor P., Srajya M. C., 1962. - Studio preliminare sulle oseillazioni libere del Golfo di Cayliari. "Ann. di Geofisica ". XV, 1.

$\left({ }^{30}\right)$ CaLor P., 1961. - Validità delle teorie idrodinamiche sulle sesse: conferme dei laghi di Levion e Caldonazzo. "Annali di Geofisica ", XIV, 3.

${ }^{31}$ ) Calol P., Romeanu G., 1965. - Osservazioni limnografiche sul lago di Albano. "Ann. di Geofisica", XVIII, 1.

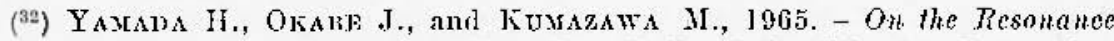
Effect in a Storm. Surge (Part I). "Bull. of the Disaster Prevention Research Institute ", 15, Part. I, N. 89, pp. 45-61.

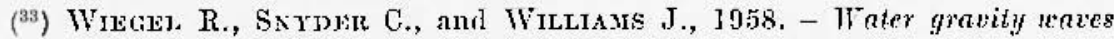
generated by a moring low pressure area. "Trans. Ameriean Geophysical Union ", 39, pp. 224-236.

$\left({ }^{34}\right)$ YaMAda H. and OKabE J., 1965. - On the Resonanee Fffect in a Stom Surge (I'art. II) "Bull. of the Disnster l'revention Rescarch Instititen, 15, Part 2, N. 94, pp. 59-74.

$\left({ }^{35}\right)$ StruvisI F., 1972. - A numerical experiment on wind effects in the Aldratic sea. "Ace. Naz. Jineei. Rend. Šc. fis. mat. e nat.", LII, 2.

${ }^{(36)}$ Donn W. I. and 1). M. Snow, 1963. - Sea Level and Climate of the Past Century, "Scienec ", N. 142, pp. $1166-1167$.

$\left({ }^{37}\right)$ Widlete II, C., 1950. - In * Centenaty Proceedings of the Royal Aleteor. Society n, (Rog. Met. Soc., Londion 1950) p. 195.

$\left({ }^{38}\right)$ Mttchw.J. M., 1961, - "Ann. N. Y. Acad. Sci. ", 95 (1), 235.

$\left({ }^{39}\right)$ Partullo Junp G., ed al., 1955. - The seasonal oseillation in sea level. "Journ. Mar. Res, ", "Sears Foundation ", 14, pp. 88-156.

$\left({ }^{40}\right)$ P'ATrum.o J. G., 1960. - Seasonal varialion in sea level in the Pacific Ocean during the International Geophysical Jear, 1957-1958. "Journ. Mar. Res.", "Sears Foundation ", 18, 1p. 168-18t.

(41) SIAw D. M. and Doxy W. $\mathrm{I}_{1}$, 1964. - Sea-level Variations at Ieelnind and Bermuda. "Journ. Mat. Res, n, 22, 2, pp. 111-122. 
(42) Puatzmasx G. W., 1972. - North Allantio Oeean: Preliminary Deserip. tion of Normal Modes. "Science n, 178, N. 4057, pp. 156-157.

${ }^{(43)}$ Pordi S., 1941. - Loseillazione anuma del Lare Yediterraneo. "Areh. di Ocean. e Limn." Anno 1, fasc. 1.

(44) Mostet F., 1969. - Te cariazioni relative del livello marino nell'Adriatico dal 1536 al 1967 e il problema dello sprofondamento di Tenezia. " Boll. Geof. teor. ed appl. ", XI, ple. 243-254.

${ }^{(45)}$ Pot.Li S., 1953. - Il graduale aunento del livello det mare lungo le coste italiane. "Geofisica pura ed appl, ", 25, pp. 123-129.

${ }^{(46)}$ Calor P., I967. - Sui jenomeni di anomale abbassantento del suolo, eon particolare riguardo al Della P'adano. "Ann. di Géofisica ", XX, 4, p's. 331. 400.

(47) CaLor P., 1970. - Come la natura rengisen all'intervento dell uomo. Responsabilitì di chi proroca $c$ di chi interpreta tali reazioni. "Ann. di Geofisica ", XXIII, 4, pp. $247-305$.

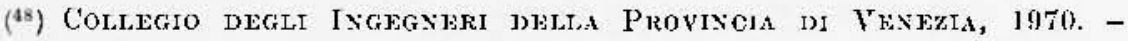
Sul Problema di Tenezia, Venezia (Dicembre).

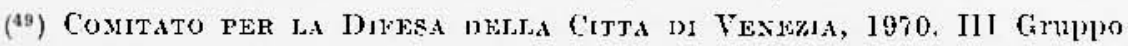
di lavoro, Relazione del sollogruppo per lo studio degli abbassamenti iel suolo.

${ }^{(50)}$ Mosetri F., 1969. - Relazione fra la frequenza dell aequa alla e l'awmento del livello medio marino a Tenezin. "Boll. Geofis. teor. "appl.", XI, p). $255-260$.

(51) Supixo G., 1970. - Soma la marea nella lagma di Tenezia. IBologna.

(52) Spagnuolo 0., 1970. - Tenezia, Tecnica e Tecnici: bradisismo di Savi. Palova.

(53) Pisexti P. e Rosa Sarva l', 1972. - Ut saera aestuaria urbis ef libertatis sedes perpetuam onscrentur. "Ist. Ven. Se., Let. ed Arti ". Estratto dal vol. V * Rapporti tella Commissione studio provved. conserv. dif. Iaguna e citti Venezia $n$. Venezia.

(54) Pinazzori l', 1970. - Alounc schematizazioni per landamemto del livello medio marino a Venezia. "Tenica Italiana", XXXV, N. 1-2, pp. 1-8.

${ }^{55}$ ) PoLL1 S., 1947. - Analisi periodale delle seric dei livelli marini di Trieste $e$ Venezia. "Gcolis. pura ed appl. ", X, 1-2.

(56) Caputo M., Pinrt I., Rossi J'jei F., 1972. - Kand subsidenee in Tenice and Porto Corsini. "Ann. di Geofisica ", XXV, 1.

${ }^{(57)}$ Piccori A., 1972. Esame delle piene verificatesi nel Novembre 1966 e loro confronto con precedenti analoghi eventi. In a $\Lambda$ tti del Converno Intern. sul tema: "P'ienc: loro previsione c lifesa del suolo", tenuto tra il 23 e il 30 Nov, 1969 ", "Acc. Naz. Lineei " Quaderno N. 169.

$\left.{ }^{58}\right)$ DORIGo I., 1968 - Le alte maree eccesiomali a Tenesia (Periodo 1\$67-1966). "Uff. Jarogr. Magistr. alle Acque". P’ubbl. N. 156, Veneria. 
(50) Sarazaxi F., 1972. - L'ultimo Dogfe. "Edizioni del Borghese ", pp. 334. Milano.

("0) Leonardi P., 1956. - Origine e noturn geologica dei terreni della Pianura Ferrese. III Convegno su "Ferato e Alimentazione *. Fermara.

(61) Neumare M., 1896 - Geologia generale (Vol. I ti "Storia tlella Terra n), "Lnione Tipogr. Editr. Torino", 1. 474.

(63) De Marcur L., Geografia Fisica. "Eulitrite Fr. Vallardi ", s.d., Torino, p. 275 .

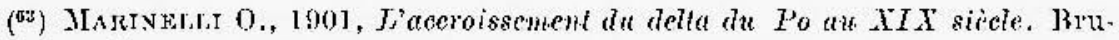
xelles.

(64) Giandotsi M., 1933. - Le piene del Po e p promedimenti di difesa. "Ace. Naz, dei Jincei, Commiss. Ttal. Studio gramli Calamition, IV, Parte I.

(56) Axdreotti G., 1938. - Sismicita del Friuli. "Atti XII Congr. Geogr. Ital. " Del Bianco * Tiline, p. 6.

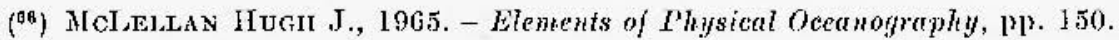
"Pergamon Press ", Oxford, 1. 20.

(67) Mosetti F., 1968. Temperatura e salinita nel follo di Trieste. "Commiss. Stmulio Oecan. e Jimm. del C.X.R. ", Serie B, N. 6, p. 6.

(68) Lisityix E., TATUUlio J. G., 1961. - The Principal Factors Infueneing the Seasonal Oscillation of Sea Level, "Jour, Geophys. Res. ", 66, pp. 845. 852 .

(69) Scaccini A., Piccinertr C., 1968. - Il fondo del mare da Catholiea a Falconara. "Commias. Stutio Ocean. Iimn. del C.N.R.", Seric C, N. 1, p. 9.

$\left.{ }^{70}\right)$ ODdone L., 1910. - Intomo alla risoluzione del problema delle sesse. "Rend. Ace. Naz, dei Jineei ", XXI.

(iI) Cinbatti XI., 1069. - Ricerehe sullevoluzione del della Padano. "Commiss. Ital. per l'Oeean. del C.N.R. ״, Serie A, N. 16. Roma.

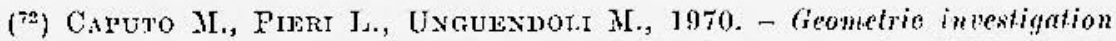
of the subsidence in the Po Della. "Boll. Geof. teor. cappl. ", XIII, N. 47, figrg. 2-5.

(73) Dorigo W., 1972. - Una laguma ai chiacchiere. "P'ro manuseripto" Venezia.

(74) Gindatti ML, Colantoxi T., 1969. - Ricerche sui fondali antistanti il Delta del Po. "Commiss. Ital, per l'Oeean. Ilel C.N.R. ", Serie A, N. 15 Roma.

(75) Debrazzr E., SEgre A., 1960. - Carta balimetriea del Hare Adriatico. " Istituto Idrografico della Marina n, fienova.

$\left.{ }^{76}\right)$ Mosettr F., Carrozzo M. T., 1971, - Some considerations on a melhod for tiles and seiches analysis. "Boll. Geof. teor. e appl.n, XIII, pls. 76-94.

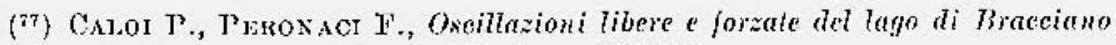
"Arch, Oecan, e Timu ", XI, 2, p. 143-160. 
(i8) CaLor P., 1972. - En ristoso caso di interazione fra atmosfera ed idrosfera: ecezionali oseillazioni del mare nel porto di Civitavecehia (17-19 Gingno 1971). "Rend. Ace. Naz, dei Lincei, Classe Se. fis., mat. c nat. ", Seric VIII, $I I I I, 1.2$.

(9) Cator P', Migaxi M., 1971, - Microsismi da piccoli bacini chiusi, da mari interni, da Oceani. "Ann. di Geofisica ", XXIV.

(89) Accerbosi F., Castbili F., Mosetti F., 1971. - Sulluso di modelli matematici idrodinamici per lo studio dellacqua alia a Tenezia. "Bholl. Geof. teor. appI. ", XIII, N. 49, pp. 18-35.

(31) Dorign L., 1901. - Maree escezionali registrate a Tenezia, Punta della Salute (Periodo 186;-1960). "Ist. Vencto Sc., Jett. ed Arti ". "Commiss. Studio Provv. Conserv. Dif. Lagma e Cittia Venezia ». Rapporti Preli. minari, I, pp. 39-69. 